\title{
Vergleich chirurgisch und radiologisch eingelegter Portkathetersysteme in einem Onkologischen Zentrum
}

\author{
Inauguraldissertation \\ zur Erlangung des Grades eines Doktors der Medizin des Fachbereichs Medizin \\ der Justus-Liebig-Universität Gießen
}

\author{
Vorgelegt von Hasan Er \\ aus Pülümür, Türkei
}

Gießen 2017 
Aus dem Institut für Diagnostische und Interventionelle

Radiologie des Klinikums Wetzlar-Braunfels der Lahn-Dill-Kliniken GmbH

Prof. Dr. med. K. Rauber

\author{
Gutachter: Prof. Dr. med. K. Rauber \\ Gutachter: Prof. Dr. med. Dr. rer. nat. F. Grimminger
}

Tag der Disputation: 26. 02. 2018 
Für Anne-Christine und Che 


\section{INHALTSVERZEICHNIS}

1 Einleitung

2 Material und Methodik.............................................................................. 3

2.1 Studiendesign, Datenerhebung und EDV-Umgebung................................................... 3

2.2 Statistische Auswertung .............................................................................................. 4



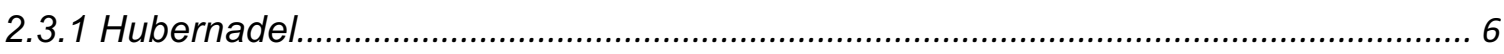

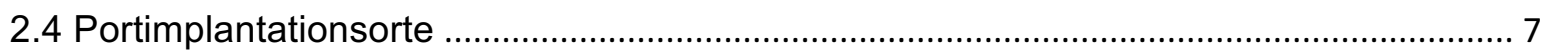



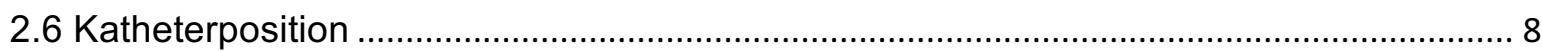



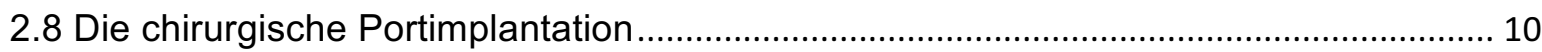

2.8.1 Technik der chirurgischen Portimplantation......................................................... 10

2.8.2 Vorteile der chirurgischen Portimplantation ......................................................... 12

2.8.2.1 Fehlen des Pneumothoracis ...................................................................................... 12

2.8.2.3 Fehlen des Pinch-off-Syndroms ........................................................................... 13

2.9 Die radiologische Portimplantation ................................................................................... 13

2.9.1 Die Technik der radiologischen Portimplantation .................................................... 13

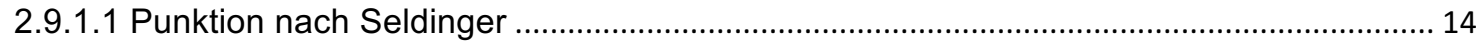

$\overline{2.9 .2 .2 ~ S u b k u t a n e}$ Einzelknopfnaht ...................................................................................... 22

2.9.2 Vorteile der radiologischen Portimplantation ........................................................ 25

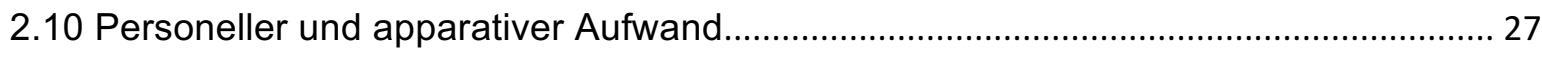

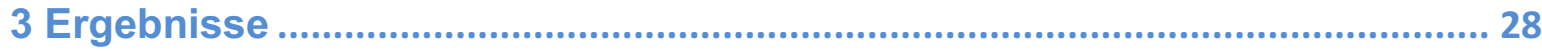

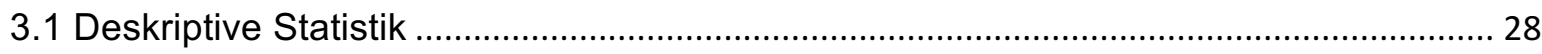

3.2.1 Tumorentitäten und Häufigkeiten in der Chirurgie ................................................ 31

3.2.2 Tumorentitäten und Häufigkeiten in der Radiologie................................................ 32

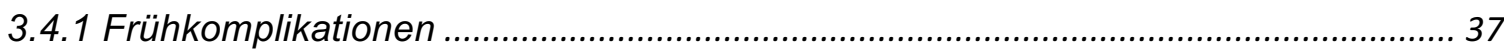

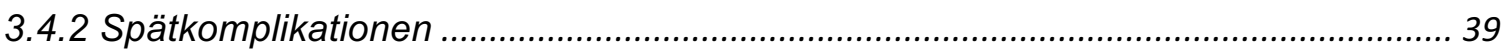

3.4.3 Radiologische Frühkomplikation Pneumothorax Facharzt VS. Assistenzarzt ........ 40

4 Diskussion ........................................................................................... 41

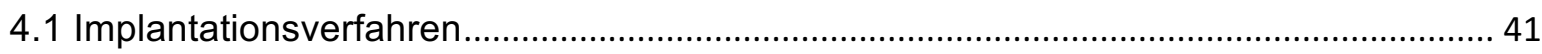

chirurgischer und radio-logischer Portsysteme ..................................................................... 41

4.1.1 Implantationsbedingte Vorteile der chirurgischen vs. radiologischen Implantation 41



4.2 Frühkomplikationen (innerhalb von 30 Tagen) …………............................................... 45

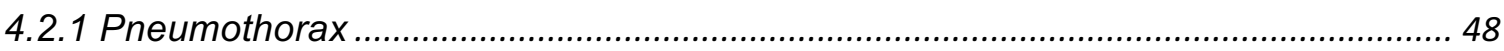

4.2.2 Pneumothorax Assistenzarzt (Punktionsnadel $21 \mathrm{G}$ ) vs. Facharzt (Punktionsnadel

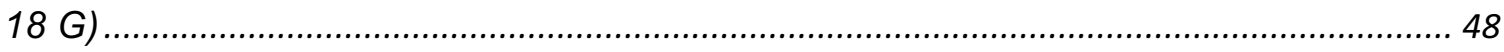

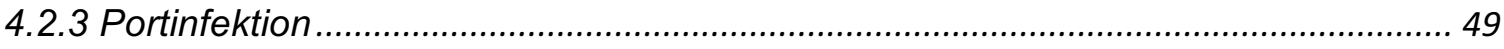

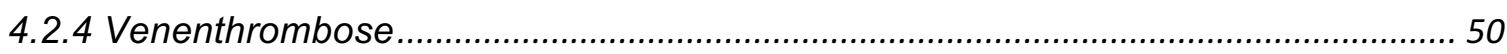


4.2.5 Encasement / Fibrinumscheidung ....................................................................... 51

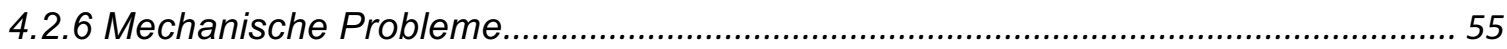

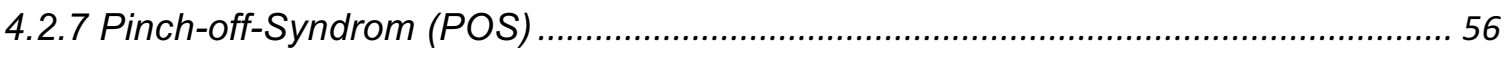

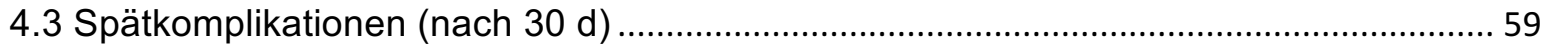

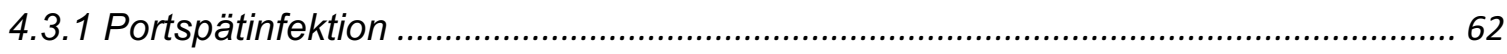

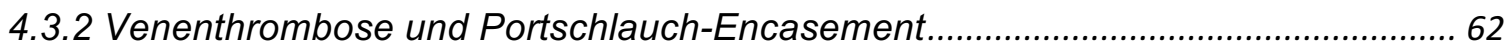

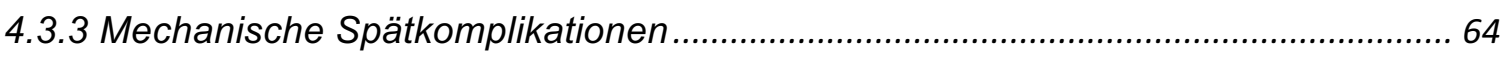

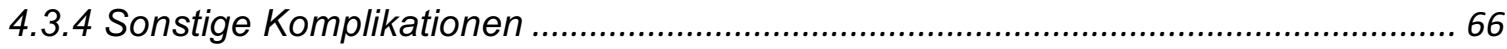

5 Zusammenfassung ................................................................. 67

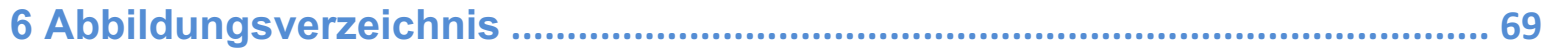

7 Tabellenverzeichnis................................................................................. 74

8 Größenangaben..................................................................................... 75

9 Abkürzungsverzeichnis ...................................................................... 76

10 Literaturverzeichnis .................................................................................... 78

11 Ehrenwörtliche Erklärung zur Dissertation ......................................... 82

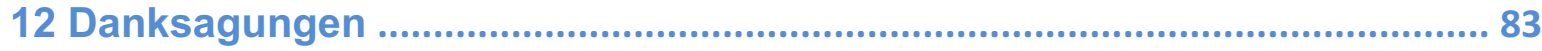




\section{EINLEITUNG}

In der Medizin sind wiederholte und langdauernde intravenöse Gaben von Medikamenten essentieller Bestandteil vieler Therapieverfahren. Bei chronisch Kranken und Tumorpatienten sind repetitive, intravenöse Zugänge für die Gabe von Chemotherapeutika, Medikamenten und parenteraler Ernährung notwendig, welche häufig zur Patiententraumatisierung führen. Viele Chemotherapeutika führen bei Applikation über periphere Venen bei geringem Venenquerschnitt und einem langsamen venösen Blutfluss zur lokalen Venenreizung und zum Veröden derselben. Folglich muss bei erneuten Punktionen auf verbleibende und oft immer weniger geeignete Venen zurückgegriffen werden.

Sichere, schnelle und zuverlässige Medikamentenapplikationen sind schon für Werner Forssman Beweggründe, nach neuen Zugangsmöglichkeiten außer der transkardialen Applikation zu suchen. 1929 gelingt inm die Sondierung des rechten Herzens mittels eines 4 Charrier Ureterkatheters [5]. Bis zu den ersten TIVADs (Totally Implantable Acces Device, komplett implantierbare Portkathetersysteme) vergehen allerdings noch 5 Jahrzehnte. Mit der erfolgreichen Implantation eines Portkammersytems gelang John Niederhuber 1982 in chirurgischer Technik mit Präparation der V. cephalica bei insgesamt 20 Patienten die erste langfristige Zugangsmöglichkeit zu einer zentralen Vene. Mit der perkutanen Punktionsmöglichkeit der Silikonportkammer war ein repetitiver, sicherer sowie für den Patienten weniger traumatischer, dauerhafter venöser Zugang möglich [19]. Der Vorteil der komplett subkutan implantierten Portkathetersysteme lag zum einen darin, dass sie eine höhere kosmetische Wohlgefälligkeit und einen höheren Patientenkomfort aufwiesen, zum anderen darin, dass bei ihnen im Gegensatz zu zentralvenösen Kathetern eine niedrigere Infektionsgefahr bestand, da kein Katheter durch die Haut ragte [27].

Zehn Jahre später wurde 1992 die erste radiologische Portimplantation in der Angiographie durchgeführt [18].

Heute ist die Portimplantation ein etabliertes und standardisiertes Verfahren in den Chirurgischen Kliniken und Radiologischen Instituten.

Die Hauptindikation für eine Portimplantation ist die intravenöse Tumor-Chemotherapie. Die Vorteile der intravenösen Applikation werden mit fehlendem Abbau durch z.B. Enzyme 
oder Peptide bei oraler (sublingualer oder rektaler) Einnahme oder bei Einschränkungen des Gastrointestinaltraktes (z.B. Kurzdarmsyndrom) angegeben [8]. Die Notwendigkeit der Portimplantation besteht bei Tumorpatienten mit kurativem und palliativem Therapieansatz, bei denen viele und langdauernde Chemotherapiezyklen mit intravenöser Gabe notwendig sind. Zudem vermeidet die intravenöse Gabe Compliance-Probleme, wie sie bei oralen Chemotherapeutika auftreten können.

Aktuell gibt es viele Verfahren der Portimplantation (Unterschiede in Zugangsvene, Portlokalisation, bildgesteuerte oder Blindpunktion, offen-chirurgisch oder radiologischminimalinvasiv). In verschiedenen Studien werden unterschiedliche Verfahren und Zugangsmöglichkeiten verglichen.

Am Standort Wetzlar werden die Patienten des Onkologischen Zentrums für die Chemotherapie regelhaft mit einem intravenösen Port durch die Chirurgische Klinik oder das Radiologische Institut versorgt.

In beiden Abteilungen erfolgt die Implantation über die V. subclavia bzw. die V. cephalica. Der - neben dem technischen Vorgehen - einzige Unterschied ist die Seitenpräferenz. Die Chirurgische Klinik implantiert die Ports vorzugsweise rechts, um eine Verletzung des Ductus thoracicus zu vermeiden. Die radiologische Implantation der Ports erfolgt von links. Dieser Zugang über das gleiche Zugangsgefäß stellt eine gute Voraussetzung dar, beide Verfahren direkt miteinander bezüglich der periprozeduralen Komplikationen, der Frühund Spätkomplikationen zu vergleichen.

Fragestellungen bezüglich der Verfahrensweisen in der Chirurgie / Radiologie:

- Welche klinisch apparenten Frühkomplikationen (<30 d) und Spätkomplikationen (>30 d) treten in der Chirurgische Klinik und dem Radiologischen Institut wie häufig auf?

- Gibt es verfahrenstypische Komplikationen (Chirurgie / Radiologie)?

- Wie hoch ist der personelle Aufwand in der Chirurgie / Radiologie?

Wir entschieden uns bei dieser retrospektiven Studie für einen Zeitraum, in dem ein radiologischer Assistenzarzt (Interventionsanfänger) in der Portimplantation mit einer dünneren Punktionsnadel angelernt wurde, um eventuelle Unterschiede in der Pneumothoraxrate in Abhängigkeit vom Durchmesser der Punktionsnadel aufzuzeigen. 
Fragestellungen im Radiologischen Institut:

- Vergleich der Pneumothoraxrate:

Assistenzarzt (21 G-Punktionsnadel $=0,8 \mathrm{~mm}$ Außendurchmesser) vs. Facharzt (18 G-Punktionsnadel = 1,3 mm Außendurchmesser).

Bezüglich des Pneumothoraxrisikos im Radiologischen Institut wurden die von zwei Fachärzten durchgeführten Portimplantationen (mit 18 G Standardpunktionsnadel = 1,3 $\mathrm{mm}$ Außendurchmesser) mit denen eines Assistenzarztes (21 G Feinnadel = 0,8 mm Außendurchmesser) verglichen.

Hierdurch ergibt sich die Fragestellung, ob mit geeignetem Material (geringerer Durchmesser der Punktionsnadel) die Punktion auch in der Hand eines interventionell weniger erfahrenen Arztes ein schnelles und sicheres Verfahren ist.

\section{MATERIAL UND METHODIK}

\subsection{STUDIENDESIGN, DATENERHEBUNG UND EDV-UMGEBUNG}

Es handelt sich hierbei um eine retrospektive Studie. Über einen Zeitraum von zwei Jahren (01. 01.2006 - 31. 12. 2007) wurden alle Daten der Chirurgischen Klinik und des Radiologischen Instituts des Klinikums Wetzlar bezüglich einer Portimplantation aus dem Orbissystem (Krankenhausinformationssystem der Firma Agfa HealthCare) gefiltert.

Mit den Patientendaten wurde manuell auf die elektronische Akte der Patienten zugegriffen und alle portrelevanten Daten in einer Excel-Tabelle (Microsoft Office) zusammengefasst. Eine gezielte Einbestellung der Patienten zu einer besonderen Nachuntersuchung einzig zur Kontrolle der Ports erfolgte nicht. Folglich wurden nur die klinisch apparenten Früh- und Spätkomplikationen erfasst. Zu den Frühkomplikationen wurden alle Komplikationen gerechnet, die bis 30 Tage nach Implantation auftraten. Im Gegensatz zu den Spätkomplikationen (> 30 Tage) gingen wir bei den Frühkomplikationen von Komplikationen in Folge des Eingriffs aus. 


\subsection{STATISTISCHE AUSWERTUNG}

Die statistische Auswertung erfolgte durch Dr. Stefan Thörner (Dipl. Sozialwiss.) - Thörner Consulting.

Zur Anwendung kamen deskriptive Statistik, Kreuztabellen mit den zu erwartenden Häufigkeiten und der Chi-Quadrat-Test.

\subsection{PORTSYSTEM}

Portsysteme werden von verschiedenen Herstellern angeboten, es gibt sie in Kunststoffund Metallausführungen sowie in verschiedenen Größen. Neben den bei uns ausschließlich verwendeten Einkammersystemen werden auch Doppelkammersysteme angeboten, welche die parallele Infusion von zwei inkompatiblen Medikamenten erlauben.

Beispiel Kunststoff-Port: Bard Power Port巴:



Abbildung 1: Kunststoff-Portsystems der Firma Bard, bestehend aus: 1) Portkammer, 2) Klick-Konnektor, 3) Portschlauch. 
Das Portsystem liegt nach der Implantation subkutan. An die Portkammer ist der Portschlauch angeschlossen und mittels Klick-Konnektor an der Portdose fixiert. Der Portschlauch führt über die V. subclavia in die V. cava superior. Die Spitze des Portschlauches liegt zentral in der V. cava superior in Höhe des rechten Hauptbronchus (atriocavaler Übergang), der topographisch-anatomischen Grenze zwischen V. cava superior und rechtem Vorhof des Herzens.

Der Portschlauch / -katheter besteht in der Regel entweder aus Polyurethan (PUR) oder Silikon. Die Portmembran besteht aus Silikon und kann nach Herstellerangaben (pfm medical) bis zu 1000 Mal mit einer nichtstanzenden $19 \mathrm{G}$ Hubernadel $(1,1 \mathrm{~mm}$ Außendurchmesser) punktiert werden.

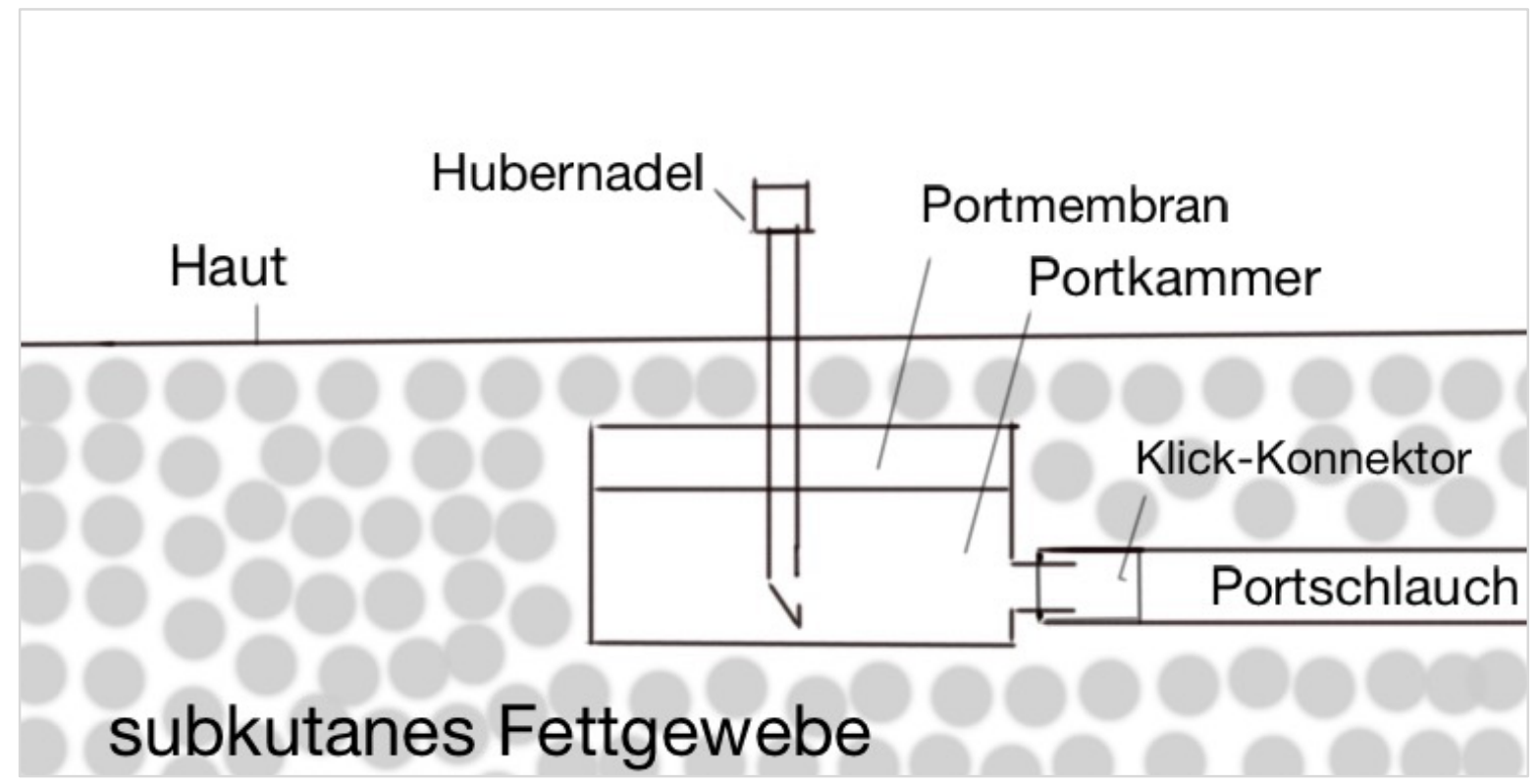

Abbildung 2: Implantierte Portkammer im Querschnitt.

Ein komplettes Portbesteck für die Implantation, wie es von verschiedenen Herstellern angeboten wird, ist unten in Abbildung 3 am Beispiel eines Portbestecks "Bard-Power Plus $\circledR^{\circledR}$ " aufgeführt. 


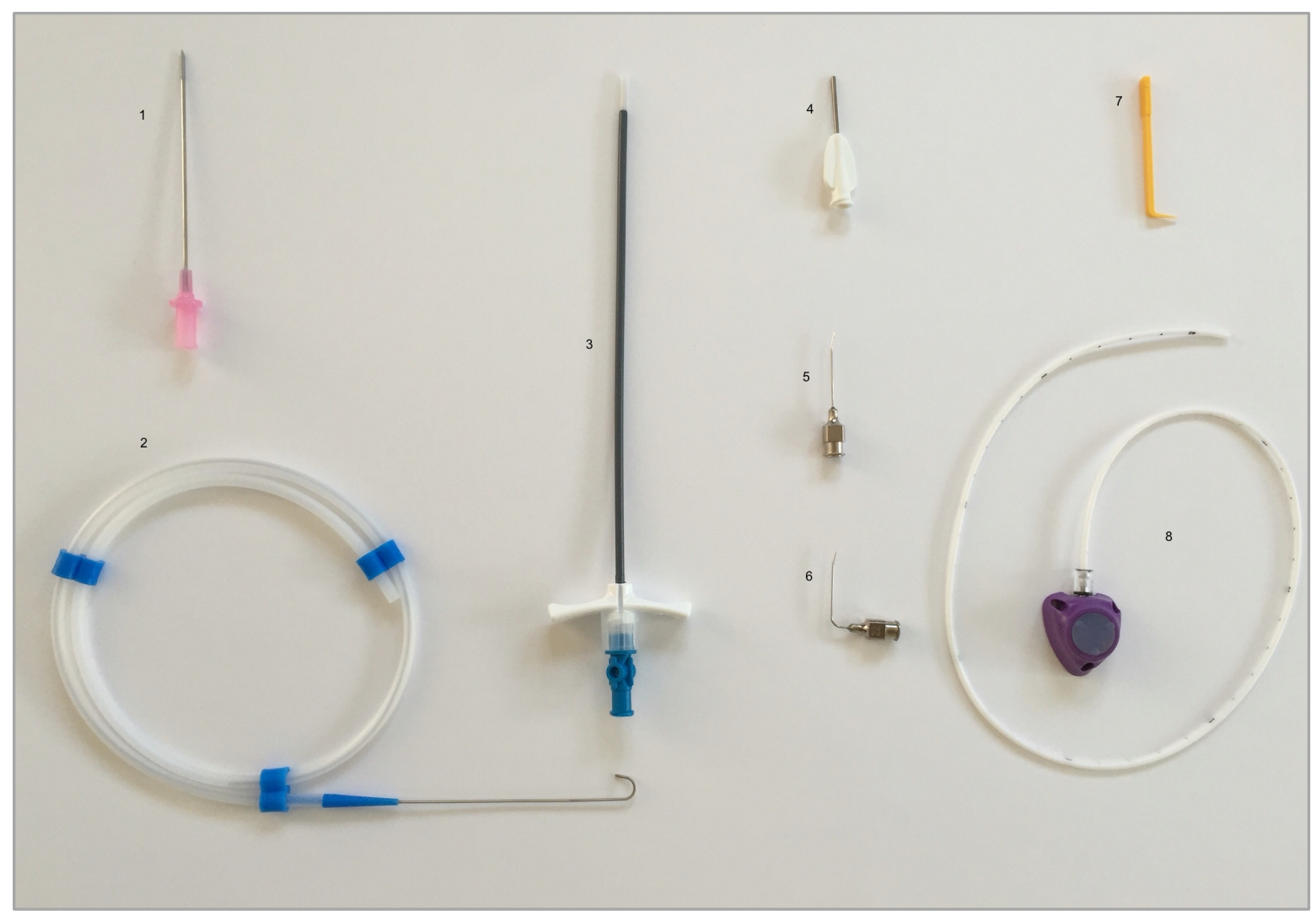

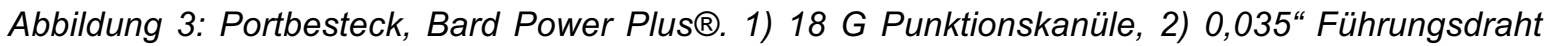
mit J-Spitze, 3) Peel-away-sheath mit Dilatator, 4) Spülkanüle, 5) Jetcan-Hubernadel gerade (22 G, $25 \mathrm{~mm}$ ), 6) Portpunktionsnadel mit Huberschliff, 7) Venenhäkchen, 8) Portkammer mit Schlauch und Konnektor.

\subsubsection{HUBERNADEL}

Die Hubernadel wurde in den 50er Jahren von John E. Niederhuber entwickelt. Sie ist eine Nadel mit Normalschliff, deren Spitze ein wenig zur Seite geknickt wurde, damit der Kanülenschliff oder das Auge der Kanüle nicht direkt auf das zu penetrierende Septum gerichtet ist [8].

Eine Punktion der Silikonmembran mittels herkömmlicher Nadel würde dagegen zu einem Stanzdefekt der Membran und damit zu einer zunehmenden Undichtigkeit führen. 


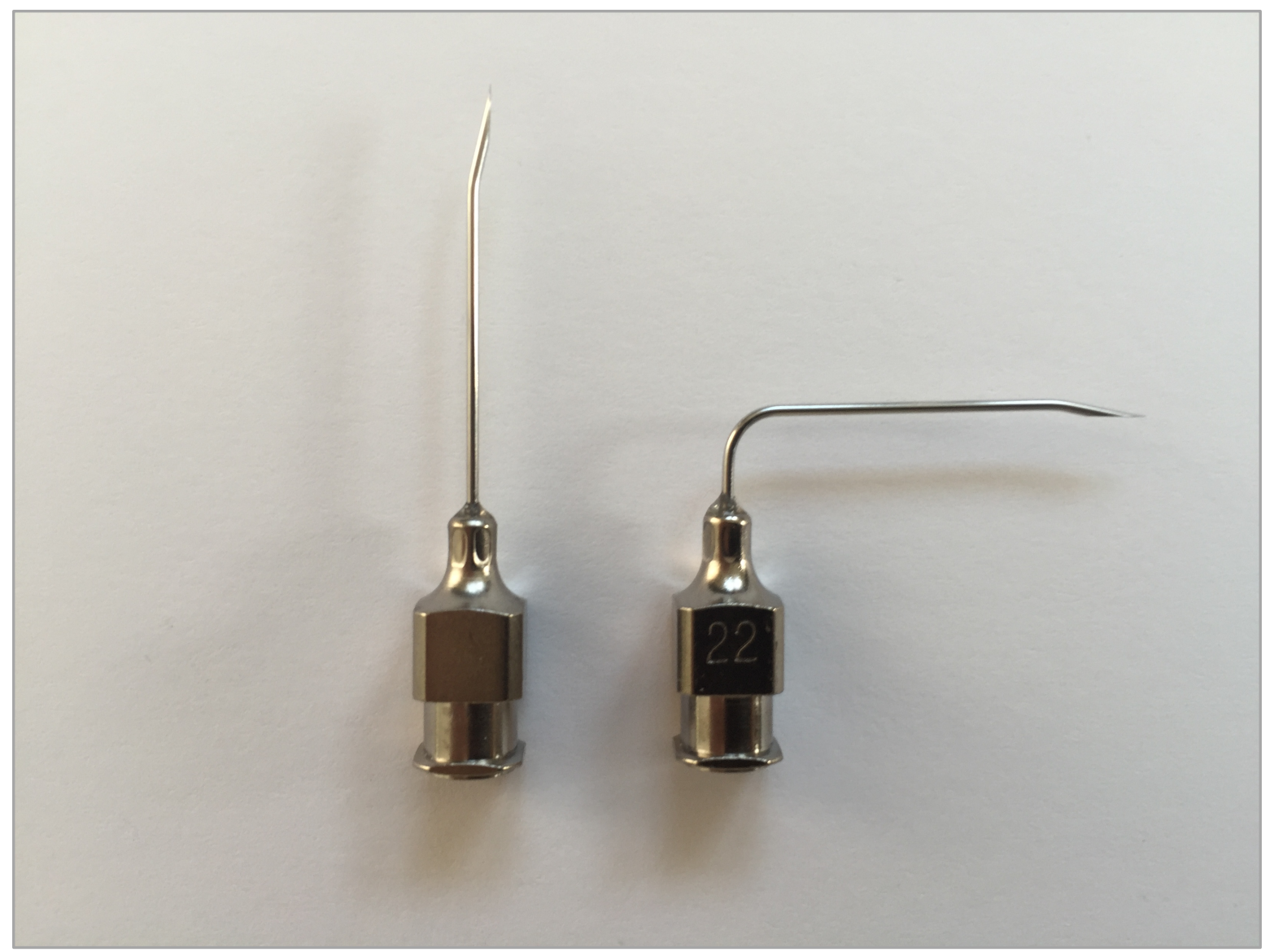

Abbildung 4: Aufgrund eines Schrägschliffs nicht stanzende Hubernadel aus einem Portset der Firma Bard®.

\subsection{PORTIMPLANTATIONSORTE}

Primärer venöser Portzugang in beiden Abteilungen ist die $\mathrm{V}$. subclavia bzw. die V. cephalica.

Bei Kontraindikationen für diesen Zugang können u. a. eine Portimplantation translumbal mittels Direktpunktion der V. cava inferior und das Tunneln des Portschlauches von rechts dorsal nach rechts ventrolateral erfolgen. Eine weitere Alternative ist die transfemorale Portimplantation via $V$. femoralis. Diese sollte aufgrund erhöhter Infektionsgefahr nur in Ausnahmefällen verwendet werden [3]. 
Zudem wäre die V. jugularis interna ein alternativer Zugangsweg, dieser wird jedoch von uns aufgrund des kosmetischen Ergebnisses mit Verlauf des Portschlauches über die Klavikula nicht genutzt.

\subsection{PUNKTIONSMETHODEN}

Die Möglichkeiten des venösen Zuganges:

- ohne Bildgebung:

○ Blindpunktion anhand anatomischer, äußerer Landmarken (Klavikula).

- Chirurgischer Cutdown.

- mit Bildgebung:

- Ultraschallgesteuert.

- Durchleuchtungsgesteuert nach Venographie.

○ Durchleuchtungsgesteuert unter Landmarkorientierung.

\subsection{KATHETERPOSITION}

Die Zielposition des Portschlauches bei Zugängen von zervikal, brachial und der oberen Thoraxapertur sollte zentral in der V. cava superior liegen (in Höhe rechter Hauptbronchus / atriocavaler Übergang). Die im Klinikum Wetzlar eingelegten Ports der Chirurgischen Klinik und des Radiologischen Instituts wurden mit der Portschlauchspitze so eingebracht, dass sie möglichst in Projektion auf den rechten Hauptbronchus enden.

Marcy empfiehlt die Positionierung der Katheterspitze ca. zwei Wirbelkörper kaudal der Carina (ausgehend von der Position des Schnittpunkts des Portschlauches mit dem rechten Hauptbronchus in der p-a- oder a-p-Aufnahme werden die Wirbelkörper nach kaudal gezählt), bei adipösen Patienten, großen Mediastinaltumoren oder einem Patientenalter über 50 Jahren sogar eine Positionierung 1 - $2 \mathrm{~cm}$ weiter kaudal der oben beschriebenen Zielposition (siehe Abbildung 5) [17]. 
Nach unserer Erfahrung macht die tiefere Positionierung des Portschlauches vor allem bei Frauen mit viel subkutanem Fettgewebe Sinn, da hier bei aufrechter Position der Patientin durch die Mammaverlagerung nach kaudal eine Verlagerung der Portkammer mit Zug des Portschlauches nach kranial erfolgt.

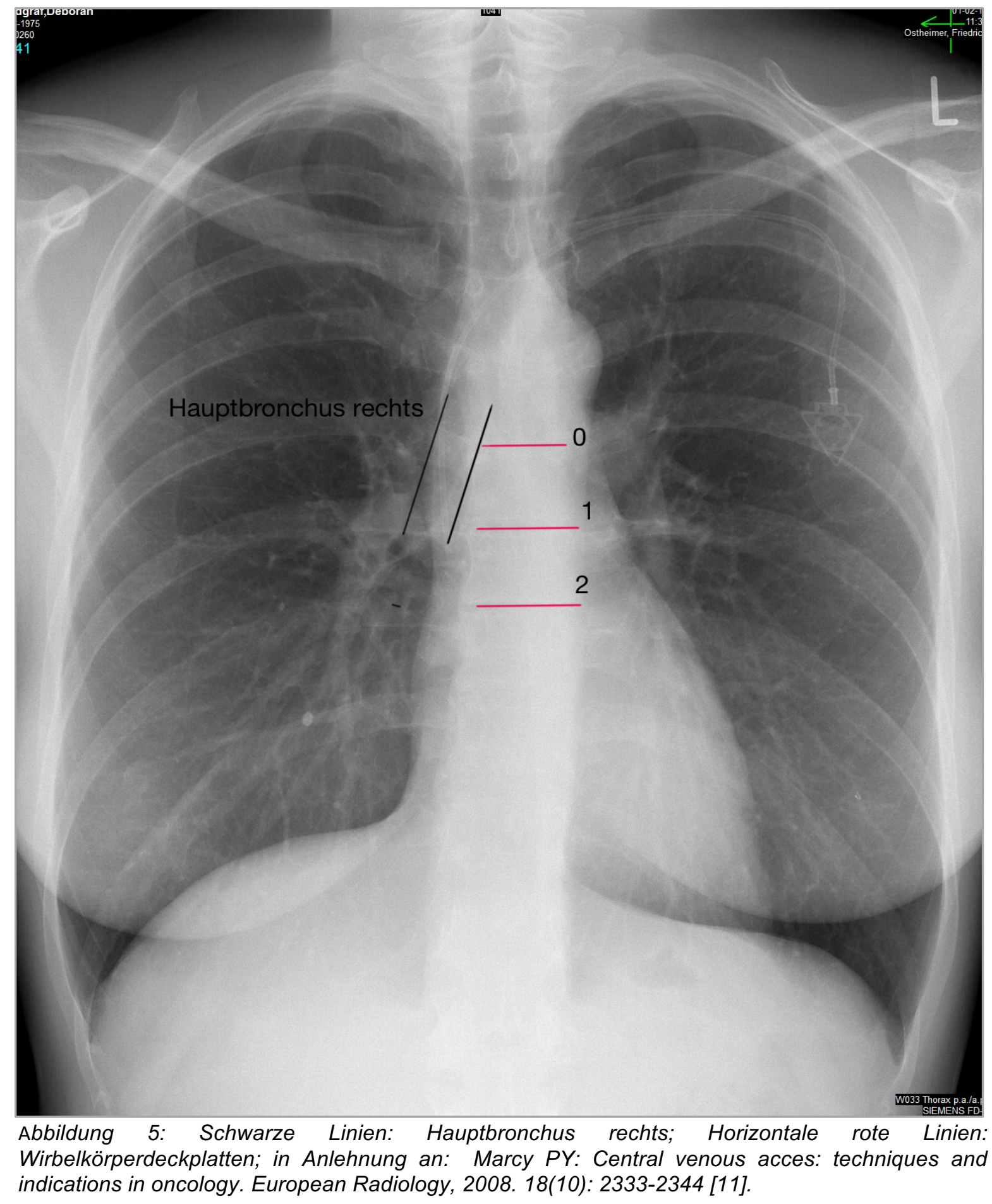




\subsection{VORAUSSETZUNG FÜR DIE PORTIMPLANTATION}

Obligat ist die schriftliche Einverständniserklärung 24 Stunden vor dem Eingriff. Bei ambulanten Patienten erfolgt die Aufklärung vor dem Eingriff.

Als wichtigstes Laborparameter wird der Quick-Wert bzw. INR-Wert herangezogen, welcher nicht unter 60 liegen sollte. Die Implantation wird auch bei einer Thrombozytopenie bis $50000 \mathrm{~T} / \mathrm{ml}$ durchgeführt. Die Laborparameter sollten nicht älter als zwei Wochen sein.

Als Kontraindikationen für eine Implantation gelten ein akuter Infekt bzw. entzündliche Veränderungen der Thoraxapertur bzw. der Haut im Bereich der geplanten Porteinlage sowie ausgeprägte Narbenbildung nach OP oder Bestrahlung. Die anamnestisch bekannte Venenthrombose gilt bei uns als relative Kontraindikation, da mit den radiologischen Methoden eine Rekanalisation oder ggf. eine Einlage über Umgehungskreisläufe grundsätzlich möglich ist (s. Abb.: 27).

Bei vorliegenden Röntgenthoraxaufnahmen sollten diese auf limitierende Faktoren bezüglich einer verminderten Gasaustauschfläche (z. B. großer Pleuraerguss, große Raumforderung, Pneumothorax) der Gegenseite überprüft werden. In solch einem Fall sollte der Port auf die ipsilaterale Seite der bestehenden Pathologie / Limitation implantiert werden, da bei einer Komplikation (z.B. Pneumothorax) "nur" der Lungenflügel mit der limitierten Gasaustauschfläche ausfällt.

\subsection{DIE CHIRURGISCHE PORTIMPLANTATION}

\subsubsection{TECHNIK DER CHIRURGISCHEN PORTIMPLANTATION}

Die chirurgische Portkatherteranlage erfolgt in der Regel unter tiefer Sedierung oder Lokalanästhesie im OP-Saal [17]. In Wetzlar werden die chirurgischen Portimplantationen ausschließlich in Lokalanästhesie und Sedation mit Anästhesie Stand-By gelegt.

Der chirurgische Eingriffsort ist stets der OP-Saal. Es erfolgt keine prophylaktische Antibiotikagabe. 
Die bevorzugte Seite der Chirurgischen Klinik für die Portimplantation ist, wie oben erwähnt, die rechte Seite, da auf der linken Seite der Ductus thoracicus entlangläuft und in den linken Venenwinkel mündet. Bei einem Cutdown rechts besteht somit kein Verletzungsrisiko des größten Lymphgefäßes.

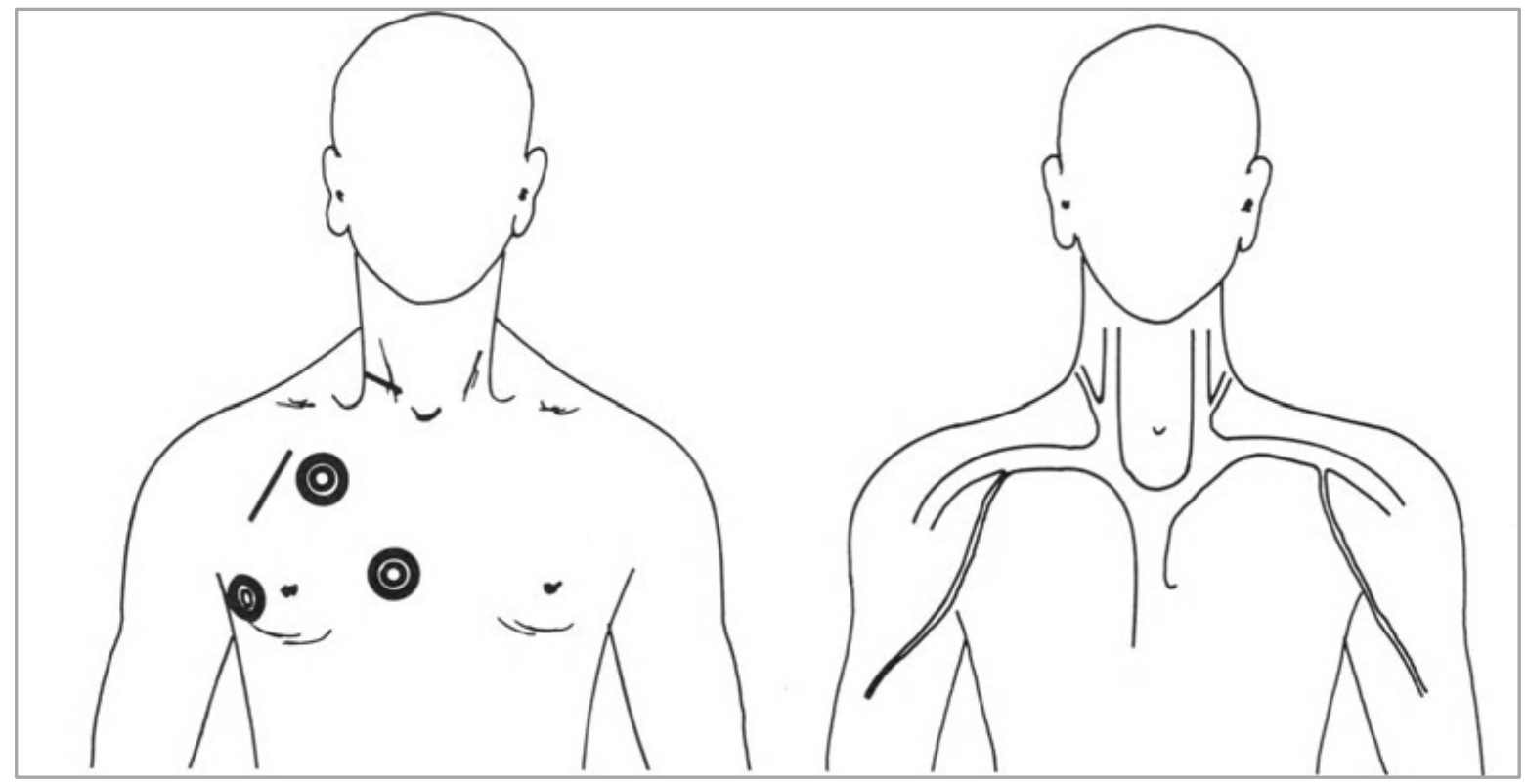

Abbildung 6: Links: Schnittführung und Platzierungsmöglichkeiten der Portkammer bei Implantation venöser Portsysteme. Rechts: Schematische Darstellung des Venenverlaufes im Schulter-HalsBereich, Haindl H, Müller H, Schmoll E, Hrsg.: Portkathetersysteme. Heidelberg: Springer; 1993: 25; With permission of Springer [8].

Nach steriler Vorbereitung des Patienten erfolgt der Cutdown auf die V. cephalica. Nach distal erfolgt eine Ligatur, nach proximal ein Anzügeln der Vene, anschließend wird die V. cephalica eröffnet und der Portschlauch mittels Venenhäkchen in die Vene eingeführt und vorgeschoben. 




Abbildung 7: Links: V. cephalica angeschlungen und eröffnet. Rechts: Einführen des Katheters in die V. cephalica. Intraoperativer Situs, aus: Haindl H, Müller H, Schmoll E, Hrsg.: Portkathetersysteme. Heidelberg: Springer; 1993: 27; With permission of Springer.

Unter Bildwandlerkontrolle wird die korrekte Lage in der V. cava superior dokumentiert und der Portschlauch gekürzt. Eine Ligatur um die proximale Vene dient der Sicherung des Portschlauches. Nach Kürzen des Portschlauches erfolgt die Konnektion mit der Portdose, welche mit Haltefäden an der Faszie des $M$. pectoralis fixiert wird. Nach einer Funktionskontrolle erfolgt abschließend die Hautnaht.

2.8.2 VORTEILE DER CHIRURGISCHEN PORTIMPLANTATION

\subsubsection{FEHLEN DES PNEUMOTHORACIS}

Der venöse Cutdown bringt eine längere Eingriffsdauer und ggf. ein Nicht-Auffinden der Vene bei adipösen Patienten aufgrund eines Verschlusses oder Okklusion mit sich [8]. Andererseits entfällt hier die Frühkomplikation des Pneumothoracis gänzlich aufgrund des Verfahrens. 
Bei chirurgischen Portimplantationen kann in ca. $10 \%$ der Fälle die V. cephalica nicht aufgefunden werden. In solchen Fällen erfolgt über Seldinger-Technik die Blindpunktion der V. subclavia [8]. Die Blindpunktion weist jedoch im Gegensatz zur durchleuchtungsgesteuerten Punktion eine erhöhte Komplikationsrate auf [6; 17].

\subsubsection{FEHLEN DES PINCH-OFF-SYNDROMS}

Ein weiterer Vorteil der erfolgreichen chirurgischen Implantation über den Zugang der V. cephalica ist das Fehlen des Pinch-off-Syndroms. Das Pinch-off-Syndrom ist die Einengung des Portschlauches in der kostoklavikulären Enge. Hierbei kann es durch eine Einengung des Katheters zu einer Obstruktion bis hin zu einer Schlauchperforation und dislokation kommen. Zum Pinch-off-Syndrom kann es im radiologischen Verfahren bei zu weit medialer Punktion der V. subclavia im kostoklavikulären Anteil kommen [12]. Dies ist beim chirurgischen Verfahren durch den Zugang über die $\mathrm{V}$. cephalica nicht gegeben.

\subsection{DIE RADIOLOGISCHE PORTIMPLANTATION}

\subsubsection{DIE TECHNIK DER RADIOLOGISCHEN PORTIMPLANTATION}

Die Portimplantation in der Radiologie findet in einem Angiographie-Raum statt und wird sowohl bei stationären als auch bei ambulanten Patienten durchgeführt.

Die Portimplantation erfolgt standardmäßig über die linke V. subclavia. Der Verlauf von der linken V. subclavia in die V. cava superior ist harmonischer. Bei Kontraindikationen wie lokaler Bestrahlung, ausgeprägter Narbenbildung, Thrombose der V. subclavia und Infektion kann die Portimplantation auf der Gegenseite (rechts) erfolgen. Auf eine standardmäßige perioperative Antibiotikagabe wird verzichtet.

Der Patient erhält am ipsilateralen Arm der Portimplantationsseite einen intravenösen Zugang, über welchen zur Sedierung $5 \mathrm{mg}$ Midazolam in einer $50-\mathrm{ml}-\mathrm{NaCl}$ Lösung per Infusion einläuft. Nach steriler Vorbereitung (Kodan $®$ Hautseptikum und Braunol®) erfolgt 
auf der gleichen Seite eine Venographie zur Darstellung der freien Durchgängigkeit der V. subclavia.

Zur Vermeidung eines Pinch-off-Syndroms (Einengung des Portschlauches in der kostoklavikulären Enge) sollte - aus beschriebenen Gründen - nicht in der kostoklavikulären Enge punktiert werden [13; 17; 34]. Wie auch in anderen Studien empfohlen sollte im mittleren Klavikuladrittel [14] oder im lateralen Drittel der Klavikula punktiert werden [17].

\subsubsection{PUNKTION NACH SELDINGER}
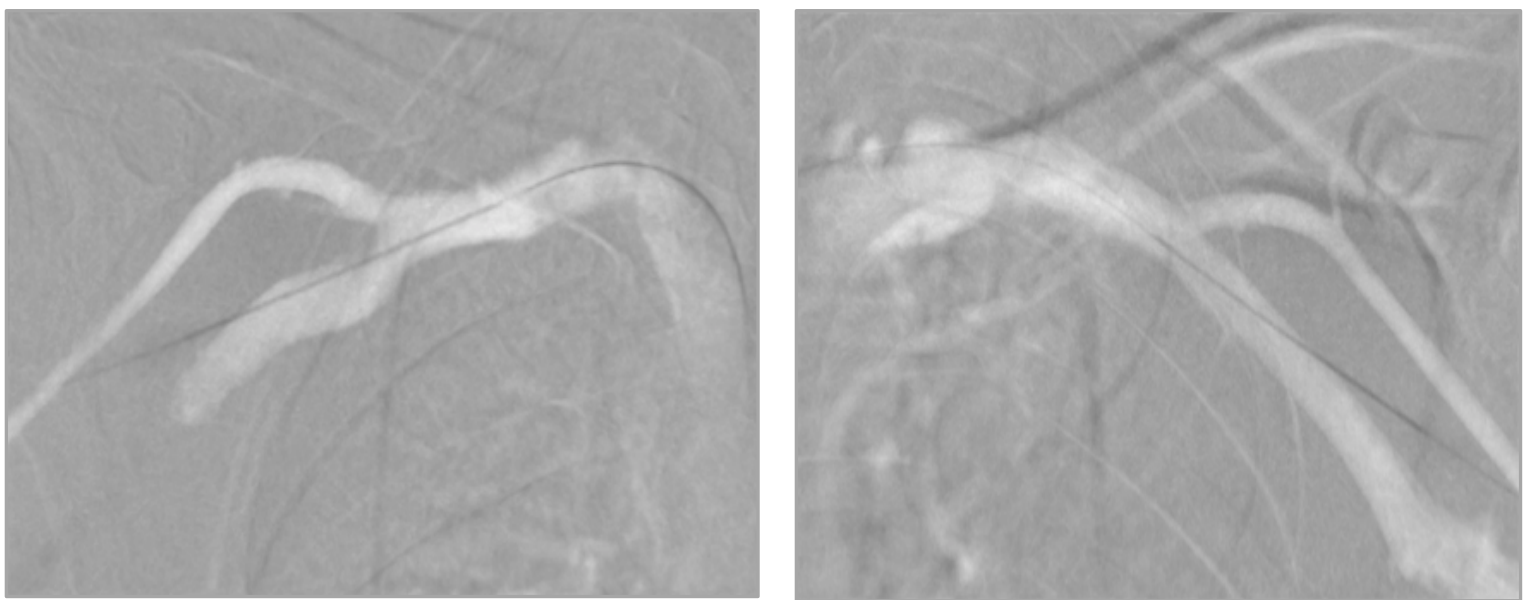

Abbildung 8: Beispiel einer Venographie rechts und links jeweils mit einliegendem 0,018 Draht.

Nach Venographie erfolgt die Punktion der V. subclavia immer in der sogenannten Seldingertechnik [23]. Je nach Untersucher gibt es verschiedene Vorgehensweisen in Bezug auf die Umsetzung. In Bezug auf unsere Studie ist dies folgendermaßen zu beschreiben:

A) Bei der von einem Facharzt ausgeführten Punktion erfolgte zunächst eine breitgefächerte Lokalanästhesie (10 ml Lidocain $1 \%$, Braun® Melsungen AG) und anschließend die Punktion der V. subclavia mittels einer $18 \mathrm{G}$ Punktionskanüle / $7 \mathrm{~cm}$. Nach erfolgreicher Venenpunktion wurde ein 0,035“ 
metallischer Führungsdraht durch die Punktionskanüle eingeführt. Es erfolgte am Punktionsort eine Stichinzision mittels Skalpell. Nach Entfernen der Punktionskanüle wurde über den Führungsdraht die Peel-away-Schleuse (auch: Split-Sheath) in die V. subclavia eingeführt.

B) Die von dem Assistenzarzt durchgeführte Punktion erfolgte mittels Feinnadelpunktionsset (Micropuncture $®$ Access Set FA. Cook, $21 \mathrm{G}$ ) und einer $10 \mathrm{ml}$ Spritze mit Lokalanästhesie. Der Hauteinstich und die Lokalanästhesie erfolgten mit der Micropuncturenadel. Unter Roadmap (Verwendung der Venographie als Maske) wurde der Stichkanal auf bzw. auch durch die V. subclavia unter lokaler Betäubung durchgeführt. Erst im Rückzug erfolgte die Blutaspiration. Bei erfolgreicher Punktion wurde ein 0,018“ Draht in die V. subclavia eingeführt (siehe Abbildungen 9 - 12). Die Hautinzision erfolgte mittels Skalpell. Die Feinnadel wurde entfernt, über den 0,018“ Draht eine Minischleuse eingewechselt, über die Minischleuse wurde nach Entfernen des 0,018“ Drahtes der 0,035“ Draht eingeführt und anschließend die Peel-awaySchleuse nach Entfernen der Minischleuse über den 0,035“ Draht in der V. subclavia platziert.

Bei beiden Punktionsarten kann durch Vorführen des Schleusendrahtes in die V. cava inferior eine arterielle Fehlpunktion weitgehend ausgeschlossen werden (Siehe Abb. 16).

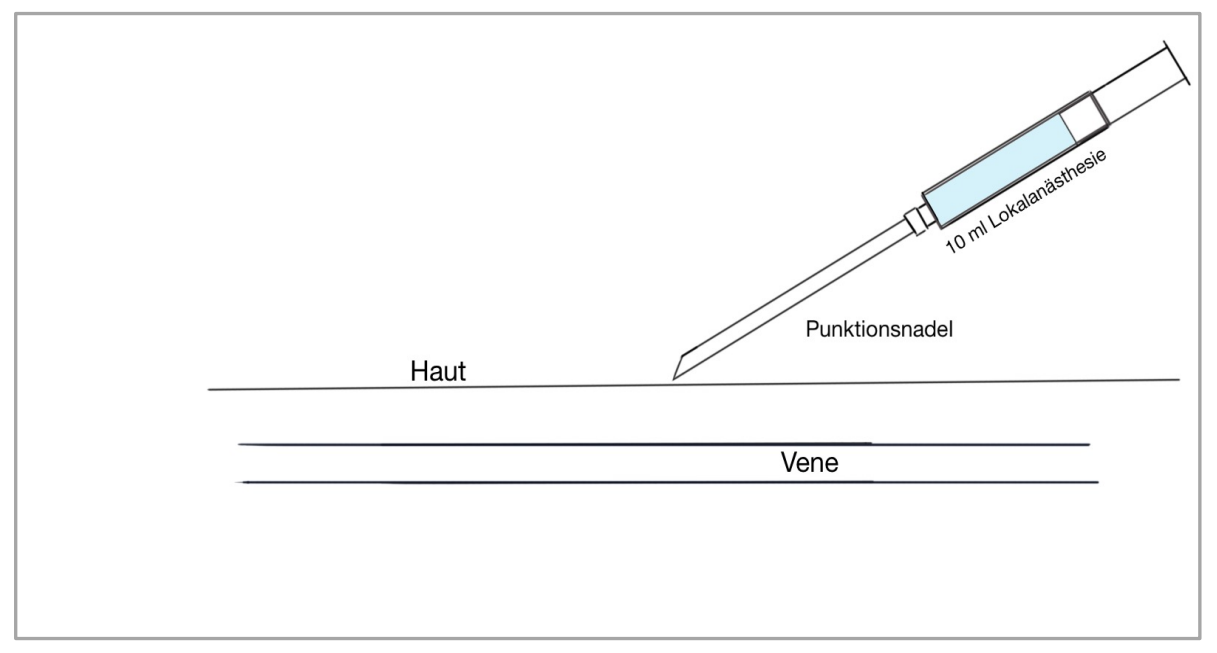

Abbildung 9: 21 G Micropuncturenadel mit 10 ml Lokalanästhesie. 


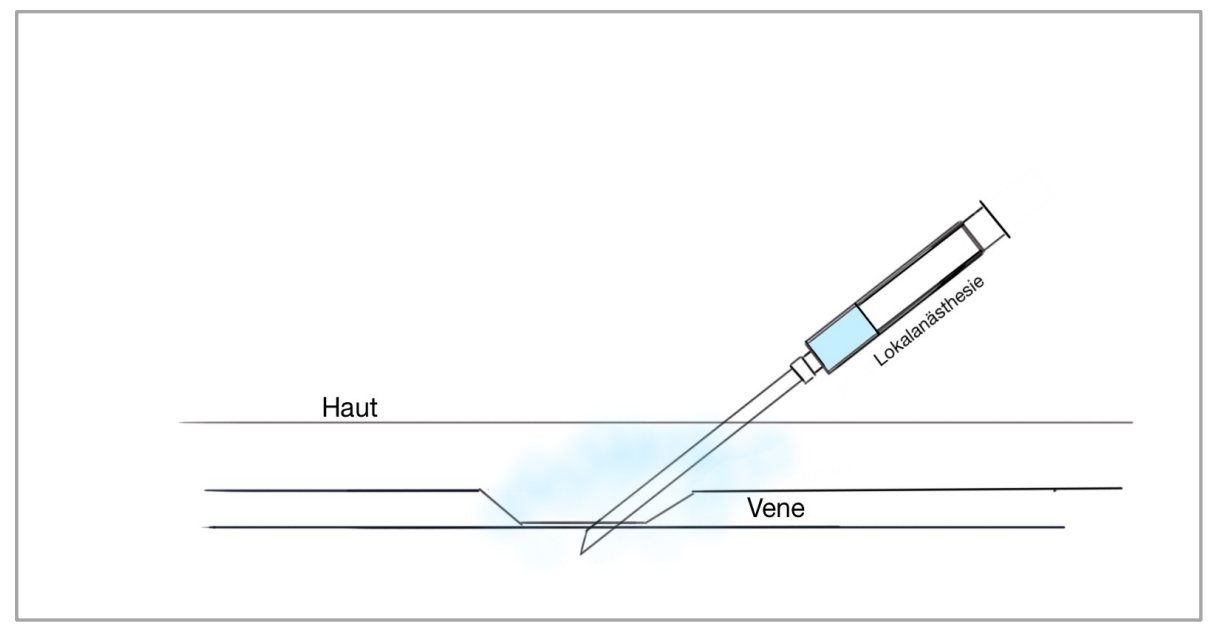

Abbildung 10: Durchführung der Lokalanästhesie mit Doppelwandpunktion.

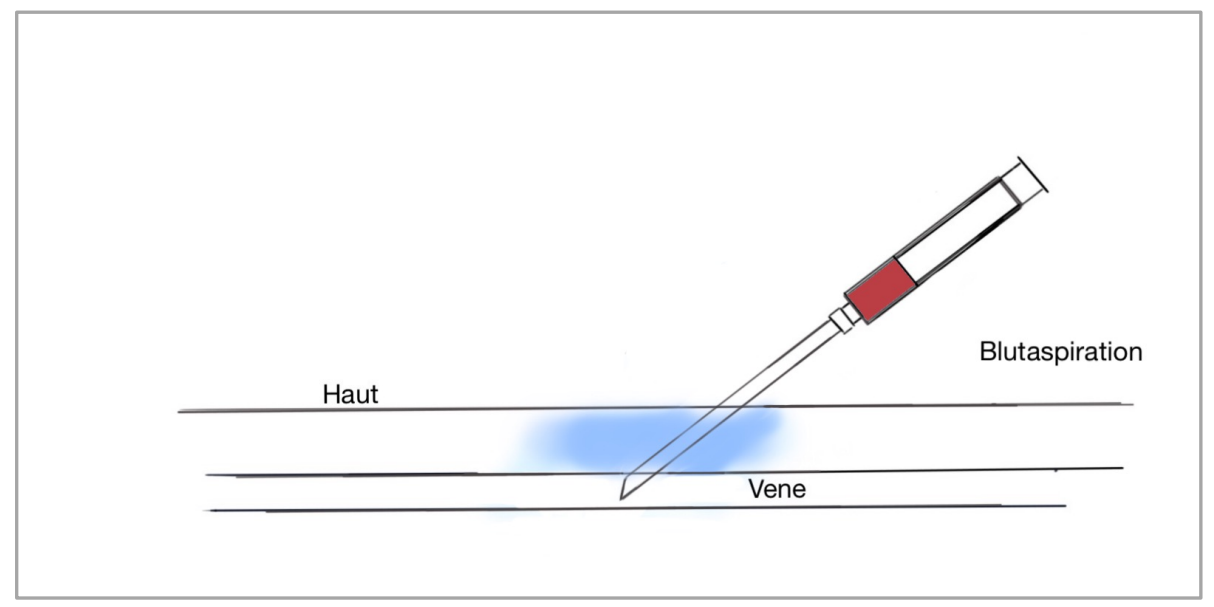

Abbildung 11: Rückzug und Blutaspiration.



Abbildung 12: Einführen des 0,018"Drahtes durch die Micropuncturenadel. 


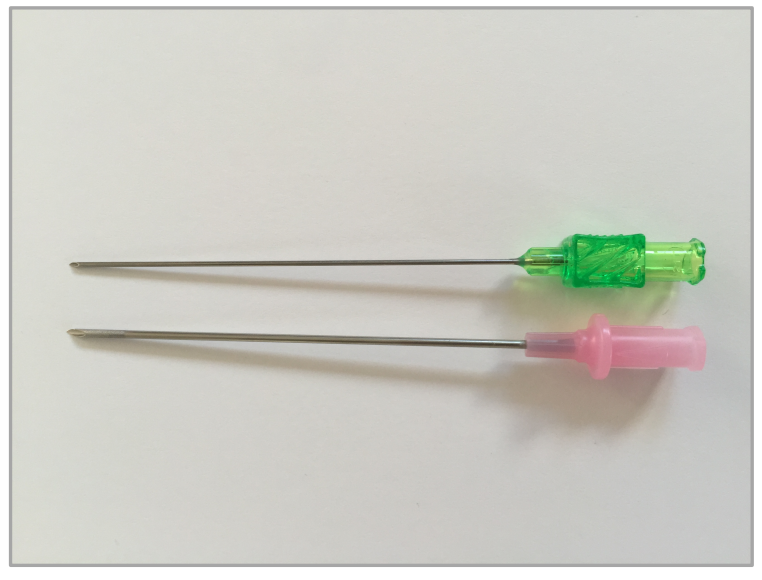

Abbildung 13: Vergleich (oben, grün), Micropuncturenadel 21 G der Firma CookMedical mit einem Außendurchmesser von 0,8 $\mathrm{mm}$, (unten rosa) normale Punktionsnadel $18 \mathrm{G}$ aus dem Portbesteckset der Firma Bard mit einem Außendurchmesser von 1,3 mm.



Abbildung 14: Micropunctureset der Firma CookMedical, USA, bestehend aus: 1.) Zweiteilige Minischleuse, $5,0 \mathrm{Fr} / 10 \mathrm{~cm}$. 2.) $21 \mathrm{G}$ Punktionskanüle. 3.) 0,018“ Führungsdraht / 40 $\mathrm{cm}$.

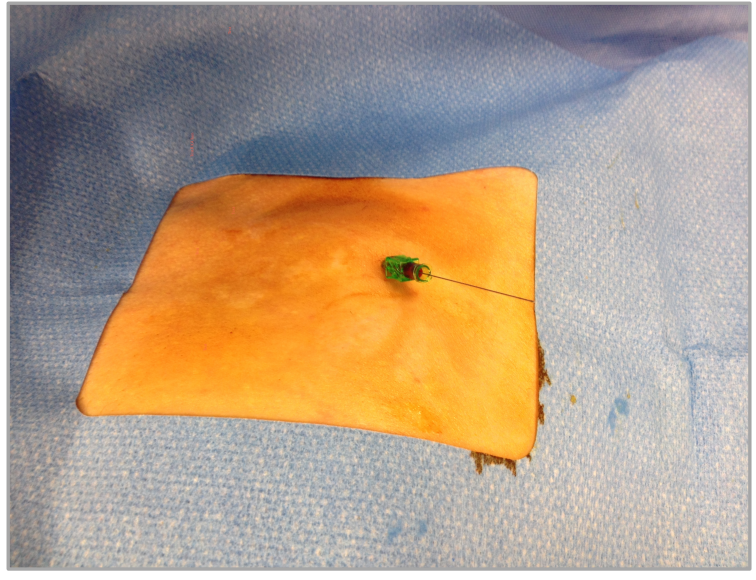

Abbildung 15: Punktion der V. subclavia mittels Feinnadel und eingeführtem 0.018“ Draht. 


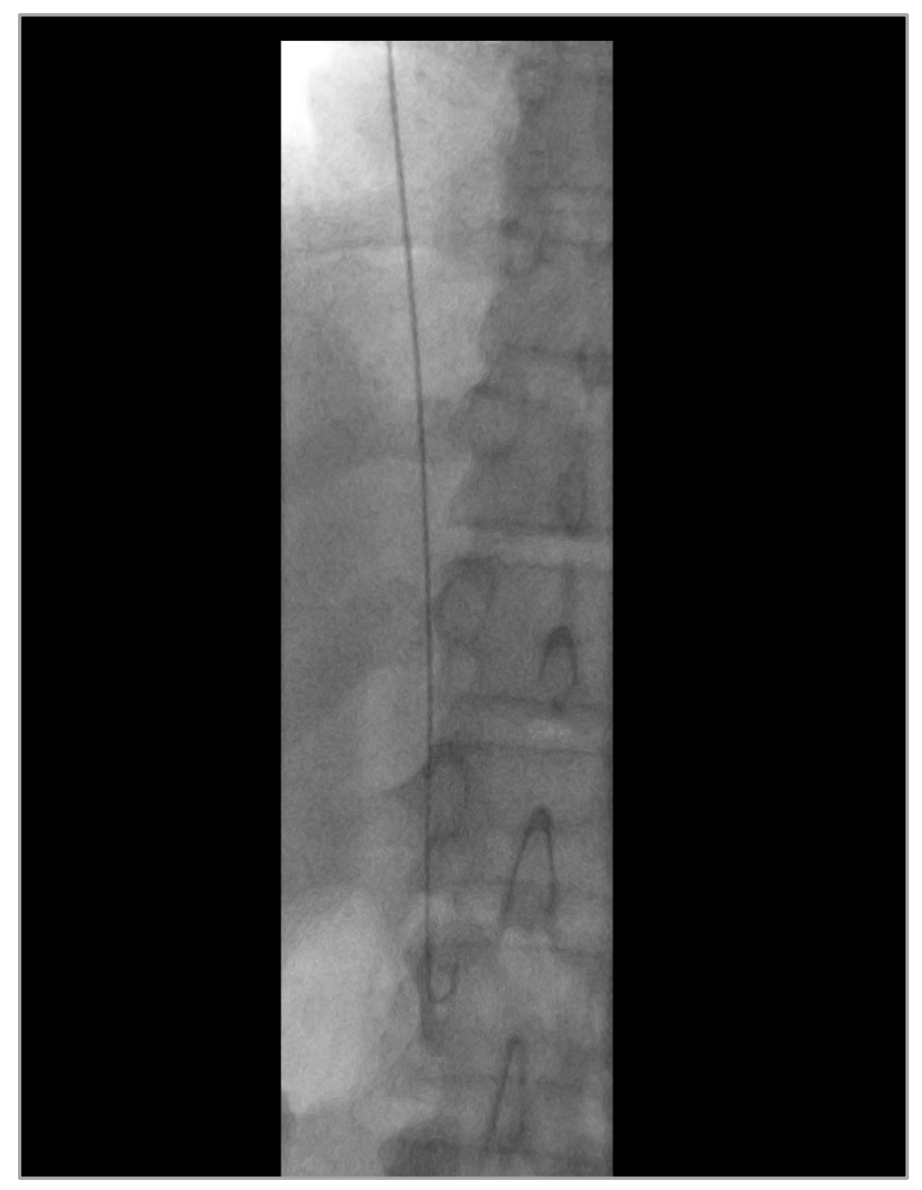

Abb.: 16: Verifizierung der venösen Punktion; der Führungsdraht liegt rechts paravertebral unterhalb des Zwerchfells in der V. cava inferior.

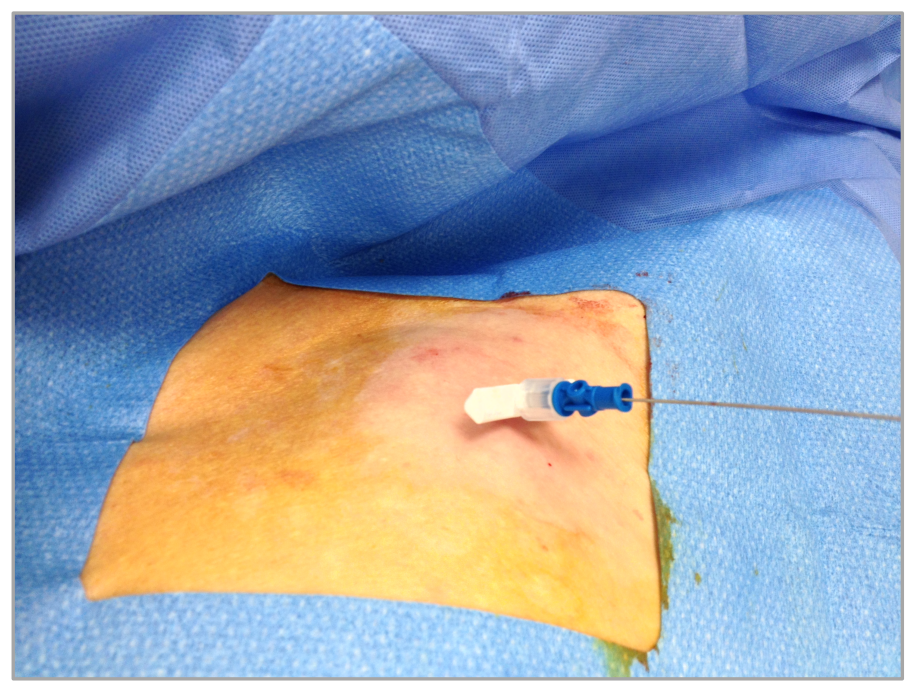

Abbildung 17: Austausch des Feinnadelsets über einen 0.035"Draht gegen eine Peel-away-Schleuse. 
Die nachfolgenden Schritte werden regelhaft wieder einheitlich ausgeführt: Ungefähr zwei Querfinger kaudal der Punktionsstelle erfolgt eine ausgiebige Lokalanästhesie (40 ml Lidocain $1 \%$, Braun® Melsungen AG). Anschließend erfolgt ein Hautschnitt von ungefähr $2-3 \mathrm{~cm}$. Mit einer stumpfen Schere wird eine subkutane Porttasche präpariert. Je nach Ausprägungsrad des subkutanen Fettes und der Körperfülle des Patienten werden zur Fixierung der Portkammer zwei Haltefäden - bei schlanken Patienten durch die Pectoralisfaszie und bei adipösen Patienten tief durch das Fettgewebe - gezogen. Bei adipösen Patienten erfolgt keine Präparation bis auf die Muskelfaszie; hier wird die Porttasche ca. $1 \mathrm{~cm}$ unter der Haut präpariert. Eine tiefere Positionierung erschwert das Ertasten und das Punktieren der Portdose. Ein mit Braunol ${ }^{\circledR}$ getränkter Tupfer wird in eine Kompresse gewickelt und in die subkutane Porttasche eingeführt. (Bei eventuellen venösen Blutungen kann hier eine gewisse Kompression erfolgen, bei arteriellen Blutungen kann mit einer Klemme abgeklemmt werden oder eine Ligatur erfolgen.) In der Zwischenzeit wird das Portsystem vorbereitet. Nach Entfernen des Brauno|®-Tupfers wird der Portschlauch mittels einer Moskitoklemme von der Porttasche aus zur Peel-awaySchleuse getunnelt.

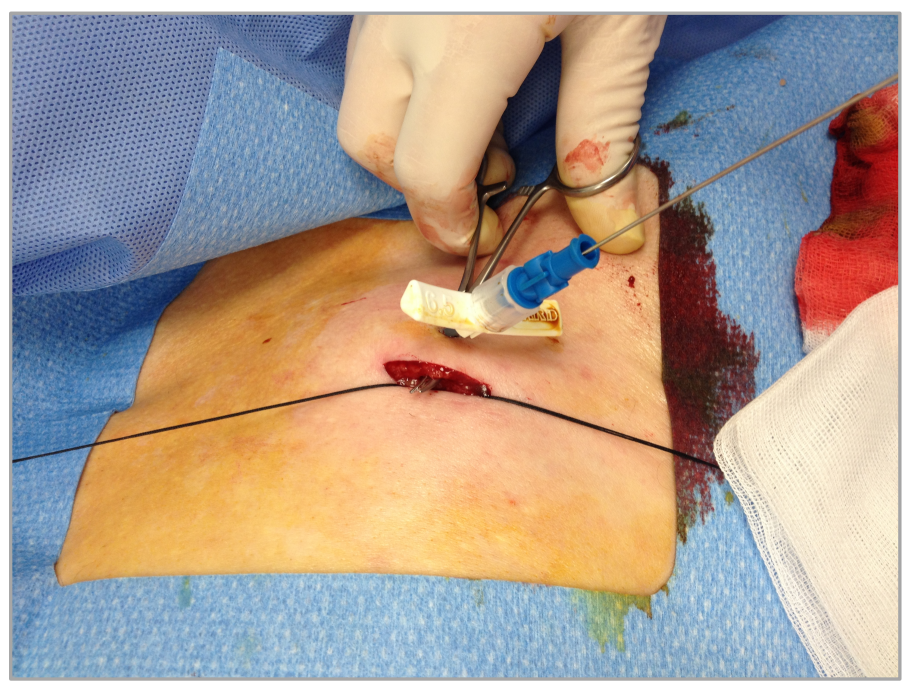

Abbildung 18: Situs mit subkutaner Porttasche und Tunnelung mittels Moskitoklemme.

Der 0,035“ Draht wird mit dem Innenleben der Peel-away-Schleuse entfernt. Dieser Schritt sollte zur Vermeidung einer Luftembolie zügig ausgeführt werden und kann auch ohne Atemkommando (Valsalva-Manöver) erfolgen. Alternativ kann mit dem Daumen die Schleusenöffnung zugehalten oder die Schleuse dicht über der Haut zugedrückt werden. 
Der Portschlauch wird durch die Peel-away-Schleuse bis in den Vorhof eingeführt. Nun kann die Peel-away-Schleuse aufgespalten und unter Rückzug weiter aufgeschält und entfernt werden.

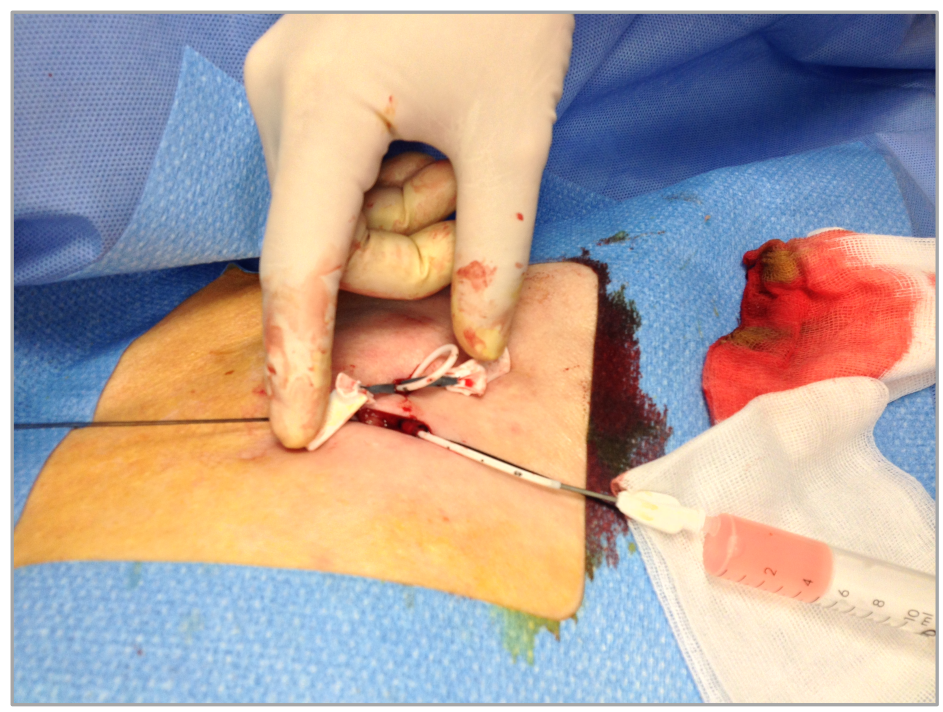

Abbildung 19: Nach Einführen des Portschlauches. Die Peelaway-Schleuse wird aufgespalten und entfernt.

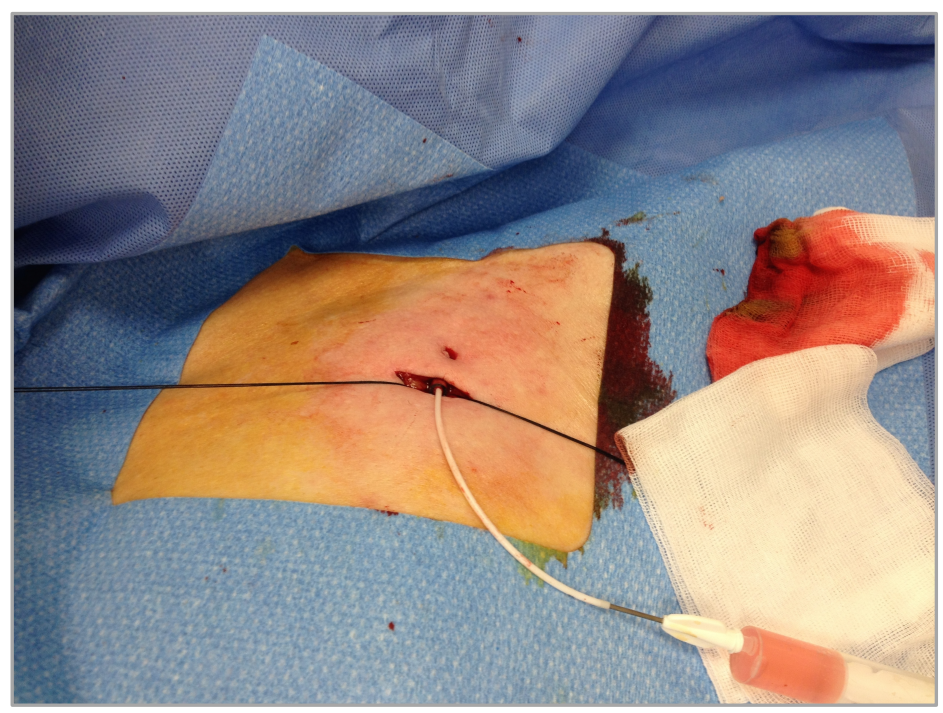

Abbildung 20: Situs nach Entfernen der Peel-away-Schleuse mit venös einliegendem Portschlauch.

Der Portschlauch wird unter Durchleuchtungskontrolle in den atriocavalen Übergang zurückgezogen und mittels Schere gekürzt. Anschließend erfolgt die Konnektion mit der Portkammer und das Arretieren des Schlauches mittels Klick-Konnektor. Die zwei 
Haltefäden werden medial und lateral durch die Ösen der Portkammer gezogen, der Port wird, ohne den Portschlauch zu mobilisieren, in die subkutane Kammer gesetzt.

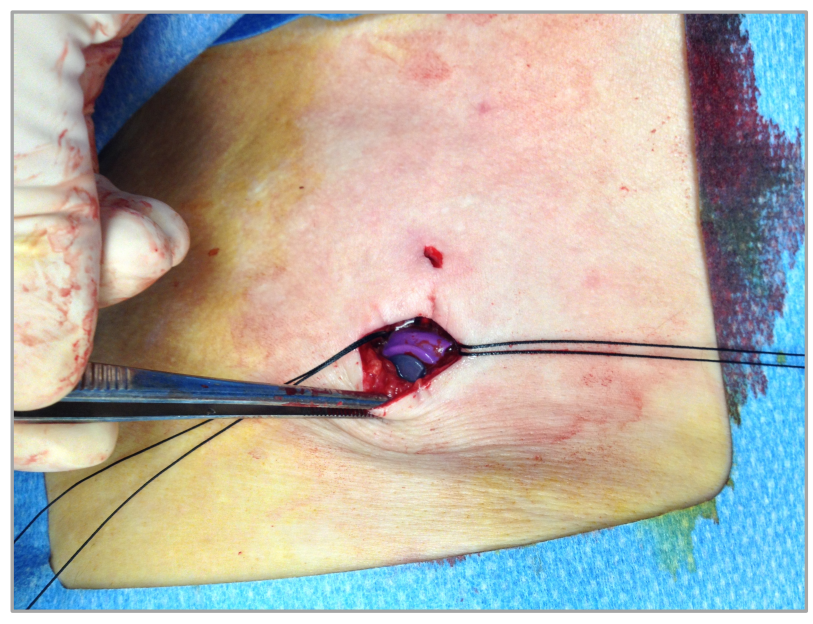

Abbildung 21: Platzieren der Portkammer in der subkutanen Porttasche.

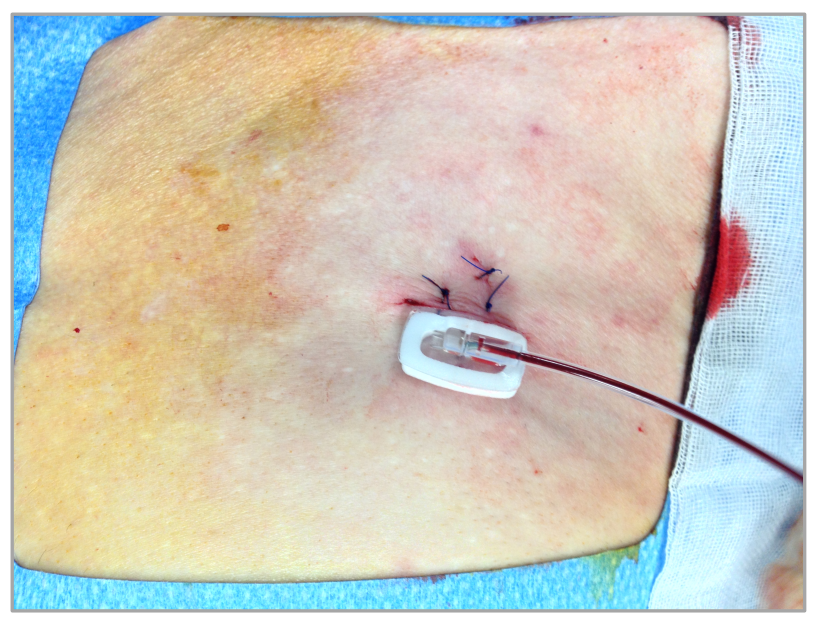

Abbildung 22: Nichtresorbierbare Einzelknopfnaht mit Portnadel mit Funktionskontrollen (Blutaspiration und Spülen).

Die Haltefäden werden geknotet und gekürzt. Anschließend erfolgt die Hautnaht und die Naht der Einstichstelle mit nicht resorbierbarem Faden mittels Einzelknopfnaht oder mit resorbierbarem Faden in subkutaner Einzelknopftechnik. Bei der Funktionskontrolle des Ports wird die Portkammer mittels Hubernadel transkutan angestochen, Blut aspiriert und

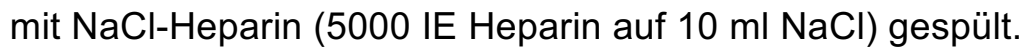


Die Dokumentation der korrekten Portlage und der Ausschluss eines Pneumothoracis erfolgen anhand eines p.a.-Röntgenbildes. Für eine anschließende Verwendung der Portkammer kann die Portnadel belassen werden. Der Port ist nach der Implantation sofort verwendbar.

Die subkutane Naht mit resorbierbarem Faden ist etwas zeitaufwendiger gegenüber der nicht resorbierbaren Einzelknopfnaht, sie erspart jedoch dem Patienten eine Wiedervorstellung in Praxis oder Klink zur Fadenentfernung. Das kosmetische Resultat ist wohlgefälliger bei ausbleibendem Strickleiterphänomen.

\subsubsection{SUBKUTANE EINZELKNOPFNAHT}



Abbildung 23: Start der Nadel im subkutanen Fettgewebe und Herausführen im subkutanen Bindegewebe.

Die Nadel wird tief in das subkutane Fettgewebe eingeführt und im subkutanen Bindegewebe ausgeführt. 


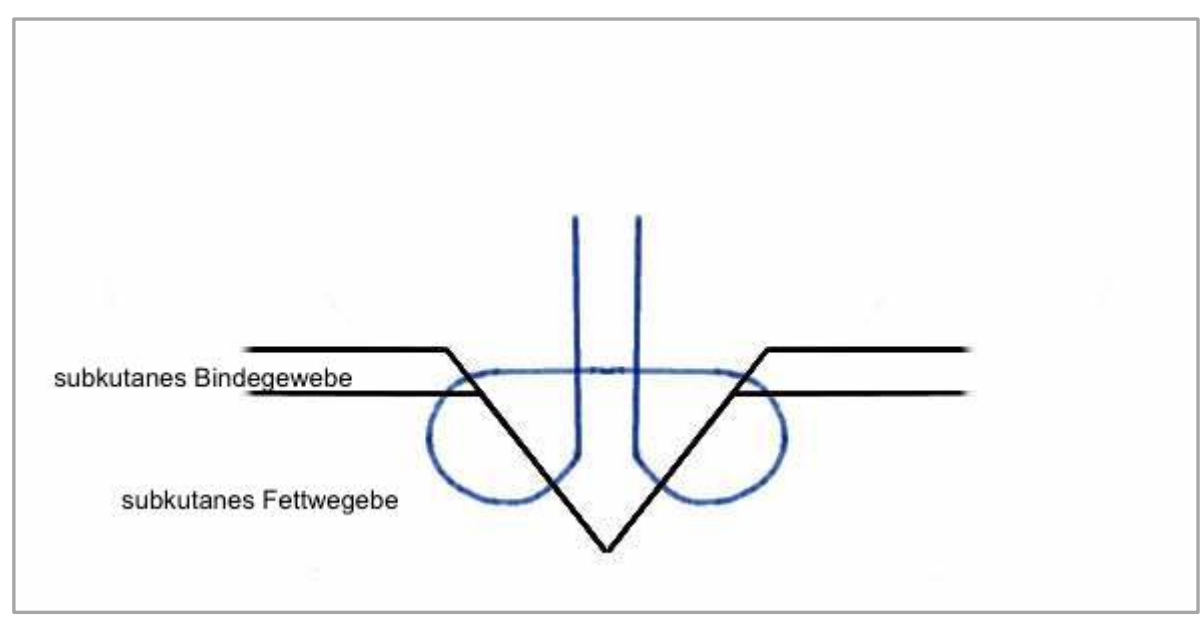

Abbildung 24: Auf der Gegenseite Durchzug der Nadel aus dem subkutanen Bindegewebe in das subkutane Fettgewebe. Beide Fadenenden werden nach oben angezogen.

Anschließend wird die Nadel auf der Gegenseite im subkutanen Bindegewebe eingeführt und im tiefen subkutanen Fettgewebe ausgeführt. Beim Verknoten werden beide Enden unter Zug gehalten.

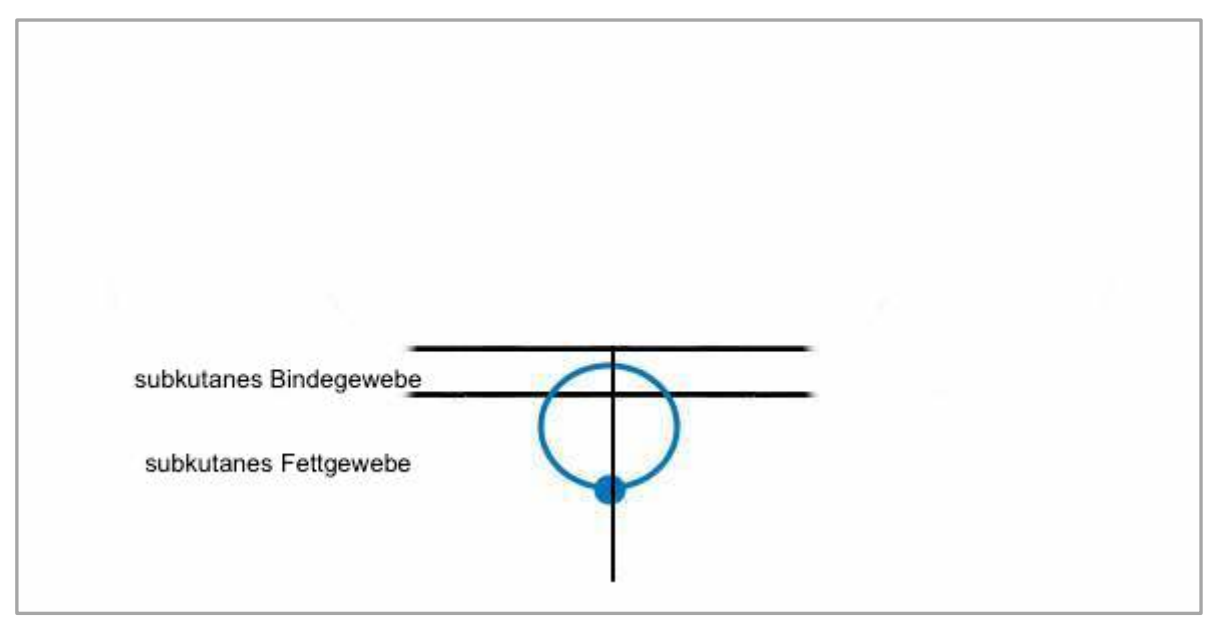

Abbildung 25: Abschlussskizze nach Knoten.

Nach dem Verknoten fällt der Knoten ins subkutane Fettgewebe und ist subkutan nicht mehr störend. Mit tiefliegendem Knoten ist die Adaption der Haut gewährleistet. Bei subkutan liegendem Knoten wäre die Hautadaption nicht gewährleistet und somit eine Eintrittspforte für Keime gegeben. 


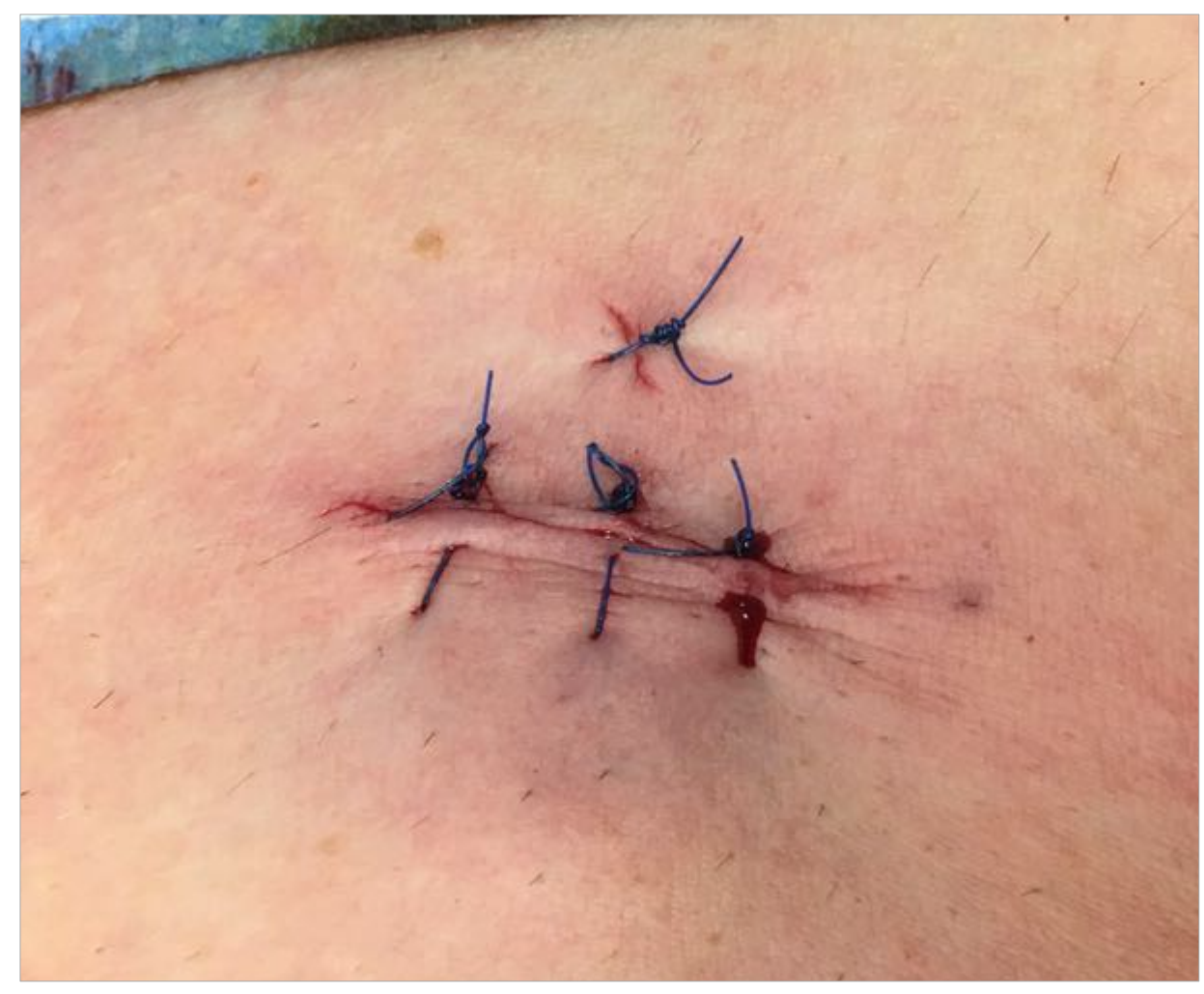

Abbildung 26: Beispiel einer nicht resorbierbaren Einzelknopfnaht (nach Donati).

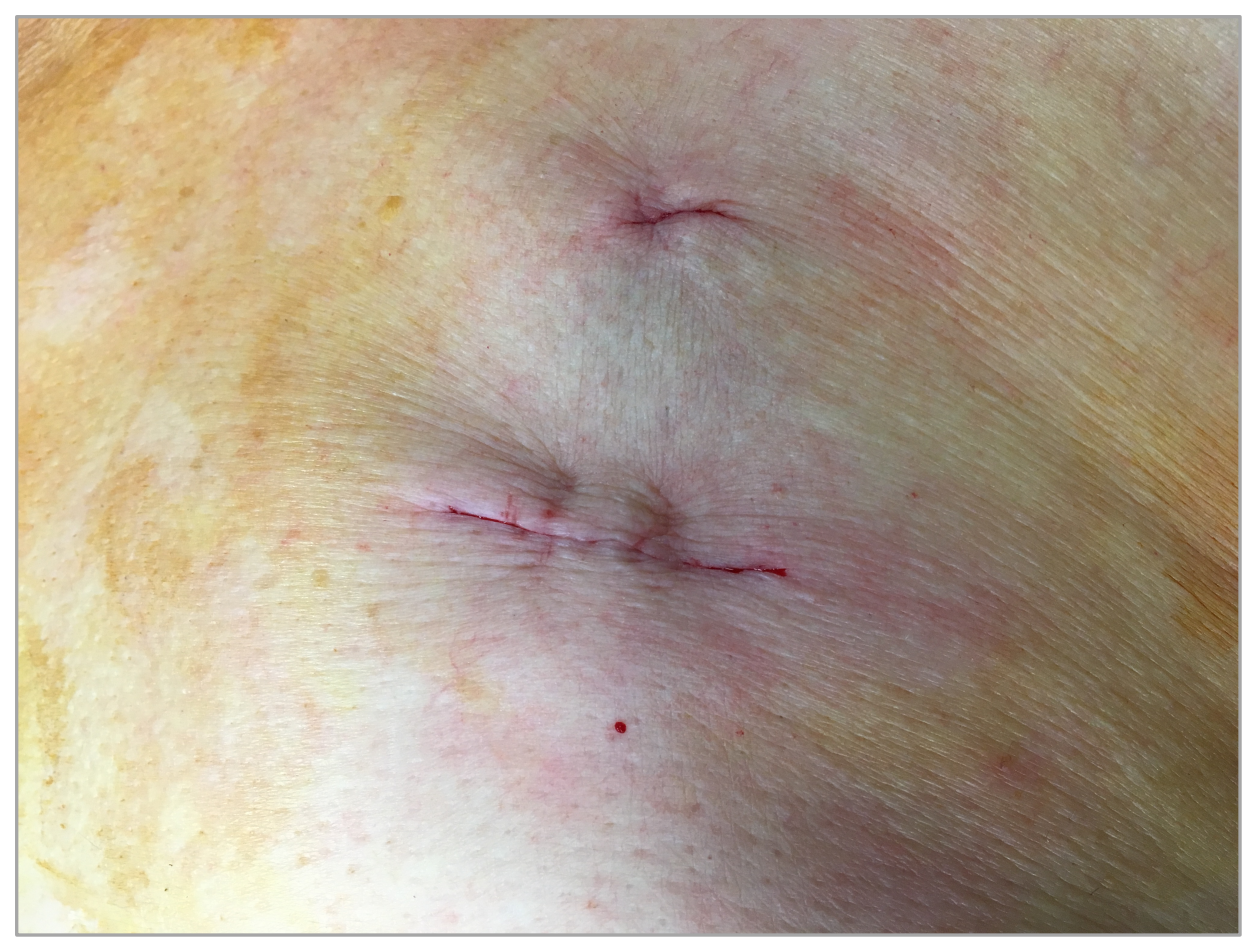

Abbildung 27: Beispiel Hautverhältnisse nach subkutaner Einzelknopfnaht. 
2.9.2 VORTEILE DER RADIOLOGISCHEN PORTIMPLANTATION

Die radiologische, durchleuchtungskontrollierte Portimplantation nach einer vorausgehenden Venographie bringt den Vorteil, dass evtl. Variationen, bestehende Normvarianten und Komplikationen erkannt und ggf. eine Adaption der Implantationsmethode erfolgen kann (siehe Abb. 27 und 28).

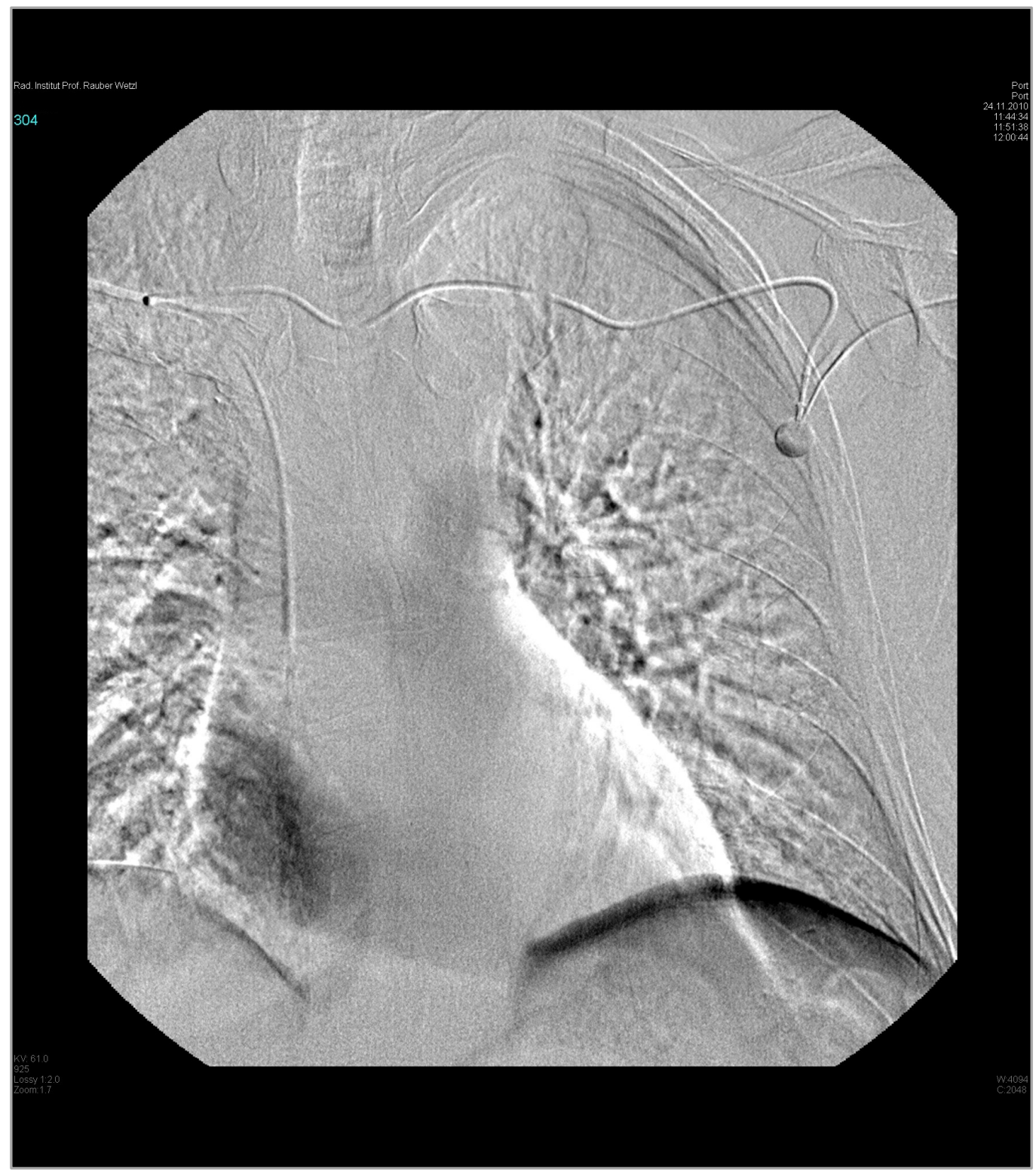

Abbildung 28: Zugang zur V. cava superior über Umgehungskreisläufe bei Verschluss der V. subclavia. 


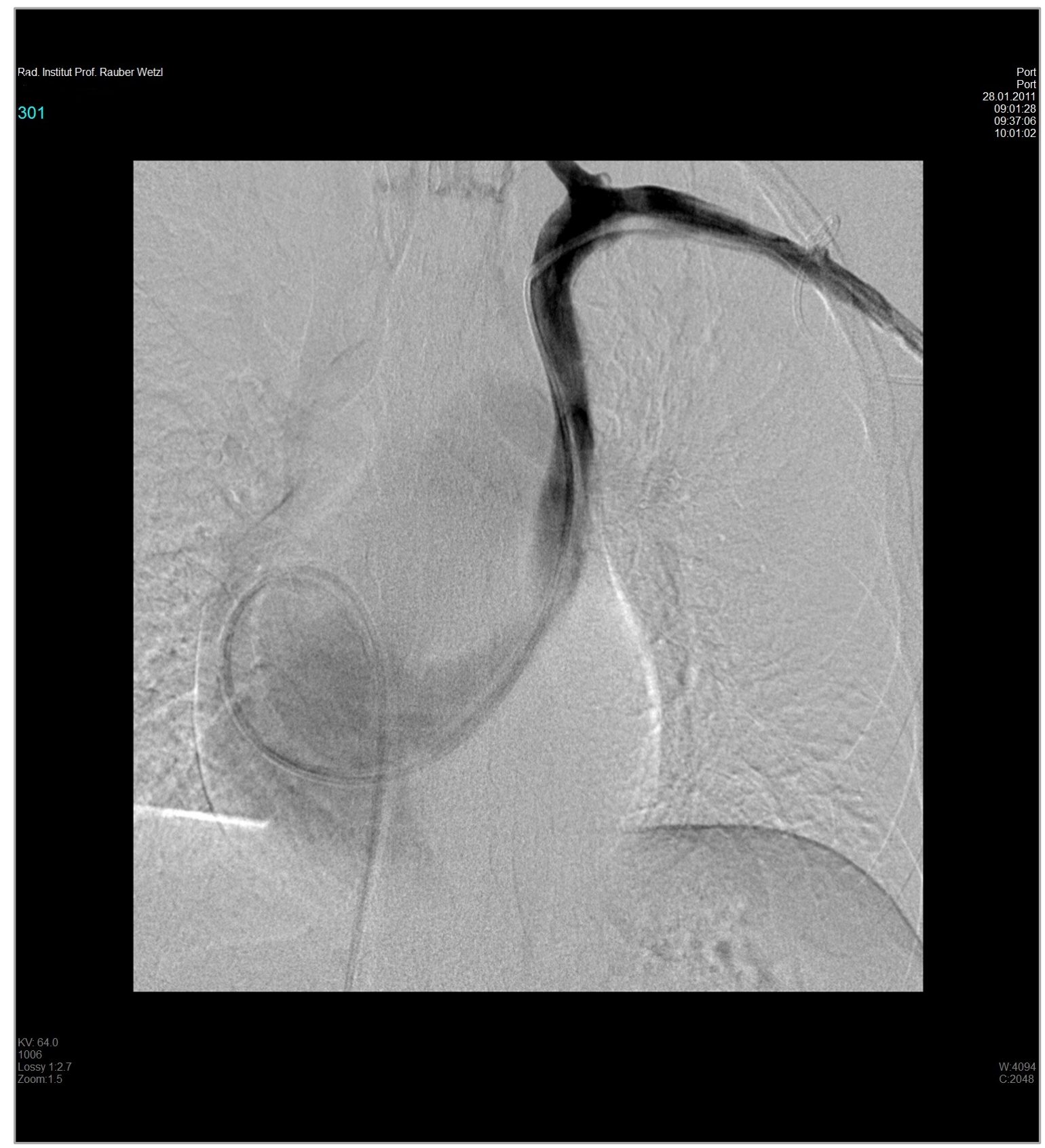

Abbildung 29: Persistierende obere Hohlvene. Nach Einlage des Portschlauches fiel der atypische Verlauf desselbigen auf. Nach Kontrastmittel-Gabe und Venographie fand sich eine persistierende obere Hohlvene. 


\subsection{PERSONELLER UND APPARATIVER AUFWAND}

Die Portimplantation in der Radiologie erfolgt durch eine Fachärztin bzw. einen Facharzt oder eine Assistenzärztin bzw. einen Assistenzarzt in der Angiographie. Hierzu wird noch eine bzw. ein MTA als unsterile Assistenz benötigt.

Der Eingriff erfolgt unter Midazolam i.v. ( bis $5 \mathrm{mg}$ Midazolam auf $50 \mathrm{ml} \mathrm{NaCl}$ Lösung als Tropfinfusion über 3 - 5 Minuten ) und Pulsoxymetriekontrolle.

Die Portimplantation in der Chirurgie erfolgt ebenfalls durch eine Fachärztin bzw. einen Facharzt oder eine Assistenzärztin bzw. einen Assistenzarzt und standardmäßig in einem Operationssaal. Zusätzlich sind eine OP-Schwester bzw. ein OP-Pfleger (Instrumentierer) und eine Springerin bzw. ein Springer mit im OP-Saal. Im Standby stehen noch eine Anästhesistin bzw. ein Anästhesist und eine Anästhesiepflegerin bzw. ein Anästhesiepfleger zur Verfügung.

Eine exakte Kostenberechnung für OP- und Angiographie-Raumzeiten existiert im Klinikum leider nicht. Jedoch ist allein durch den erhöhten Personalbedarf in der Chirurgischen Klinik von einem ebenfalls deutlich erhöhten Kostenfaktor auszugehen.

Im Vergleich zu einer radiologischen Implantation konnte in einer Studie ein erhöhter Kostenfaktor von bis zu 80 \% aufgezeigt werden, wenn die Portimplantation durch einen Chirurgen durchgeführt wurde [28]. 


\section{ERGEBNISSE}

\subsection{DESKRIPTIVE STATISTIK}

Im Radiologischen Institut wurden über die erfassten zwei Jahre 556 Ports implantiert. Zwei Portimplantationen wurden aufgrund unzureichender Dokumentation ausgeschlossen. In der Chirurgischen Klinik wurden im selben Zeitraum 173 Ports implantiert.

Die Gesamtzahl der Portimplantationen innerhalb von zwei Jahren, aufgeteilt nach Abteilungen gibt folgende Tabelle wieder:

\begin{tabular}{||l|l|l|l||}
\hline \multicolumn{2}{||l||}{ Abteilung } & Fallstatus \\
\hline Chirurgie & \multirow{2}{*}{$N$} & Gültig & 173 \\
\cline { 3 - 5 } & & Fehlend & 0 \\
\hline Radiologie & $\mathrm{N}$ & Gültig & 554 \\
\cline { 3 - 4 } & & Fehlend & 2 \\
\hline
\end{tabular}

Tabelle 1: Gesamtzahl der Portimplantationen innerhalb von zwei Jahren, aufgeteilt nach Abteilungen.

Insgesamt wurden 727 Patientendaten innerhalb der zwei Jahre erfasst. 


\begin{tabular}{||l|l|l|l|l||}
\hline \hline \multicolumn{2}{||l|}{ Abteilung } & Häufigkeit & Prozent \\
\hline Chirurgie & \multirow{2}{|l|}{ Gültig } & stationär & 173 & 100,0 \\
\hline Radiologie & \multirow{2}{*}{ Gültig } & ambulant & 168 & 30,3 \\
\cline { 3 - 5 } & & stationär & 386 & 69,7 \\
\cline { 3 - 5 } & & Gesamt & 554 & 100,0 \\
\hline
\end{tabular}

Tabelle 2: Häufigkeit der Portimplantationen nach stationär / ambulant.

Alle chirurgischen Portimplantationen wurden bei stationären Patienten durchgeführt. In der Regel handelte es sich hierbei um Patienten mit einer Erkrankung hauptsächlich aus dem Bereich des Gastrointestinaltraktes (GIT), die im Rahmen eines visceralchirurgischen Eingriffs oder während des gleichen stationären Aufenthaltes mit einem Port versorgt wurden.

$30 \%$ der radiologischen Patienten waren ambulante Patienten. Diese rekrutierten sich aus dem Patientengut der eigenen Klinik und ambulanten Patienten aus der Lungenklinik Waldhof-Elgershausen, welche uns ambulant zur Portimplantation vorgestellt wurden.

\begin{tabular}{|c|c|c|c|c|}
\hline \multicolumn{3}{|l|}{ Abteilung } & \multirow{2}{*}{$\begin{array}{l}\text { Häufigkeit } \\
60\end{array}$} & \multirow{2}{*}{\begin{tabular}{|l} 
Prozen \\
34,7
\end{tabular}} \\
\hline Chirurgie & Gültig & weiblich & & \\
\hline & & männlich & 113 & 65,3 \\
\hline & & Gesamt & 173 & 100,0 \\
\hline \multirow[t]{3}{*}{ Radiologie } & Gültig & weiblich & 287 & 51,8 \\
\hline & & männlich & 267 & 48,2 \\
\hline & & Gesamt & 554 & 100,0 \\
\hline
\end{tabular}

Tabelle 3: Häufigkeiten nach Geschlecht. 
Die Verteilung der Geschlechter zu Gunsten der männlichen Patienten in der Chirurgischen Klinik lässt sich u.a. mit der Tatsache erklären, dass der Anteil des männlichen Geschlechts mit Tumoren des Gastrointestinaltraktes an den KrebsNeuerkrankungen der Bevölkerung Hessens höher ist als der des weiblichen. Die Summe der Krebs-Neuerkrankungen des Gastrointestinaltraktes (Mundhöhle / Rachen, Magen und Darm) lagen 2008 in Hessen beim männlichen Geschlecht bei 3233 und beim weiblichen Geschlecht bei 2078 (siehe Abb. 29, Auszug aus dem hessischem Krebsregister 2008) [11]. Patienten, die aufgrund ihrer Malignomerkrankung in der Chirurgischen Klinik versorgt wurden, erhielten ihre Portimplantationen ebenfalls von den chirurgischen Kollegen. Zudem handelt es sich bei dieser Arbeit um eine retrospektive und keine prospektiv-randomisierte Studie.

Die Geschlechterverteilung in der Radiologie ist homogener, da sich das Patientenkollektiv aus einer heterogeneren Verteilung als das der Chirurgischen Klinik zusammensetzt.



Abbildung 30: Die zehn häufigsten Krebs-Neuerkrankungen, aus: Hessisches Krebsregister 2008. 


\subsubsection{TUMORENTITÄTEN UND HÄUFIGKEITEN IN DER CHIRURGIE}

Häufigkeiten nach Grundleiden der Erkrankung in der Chirurgie:

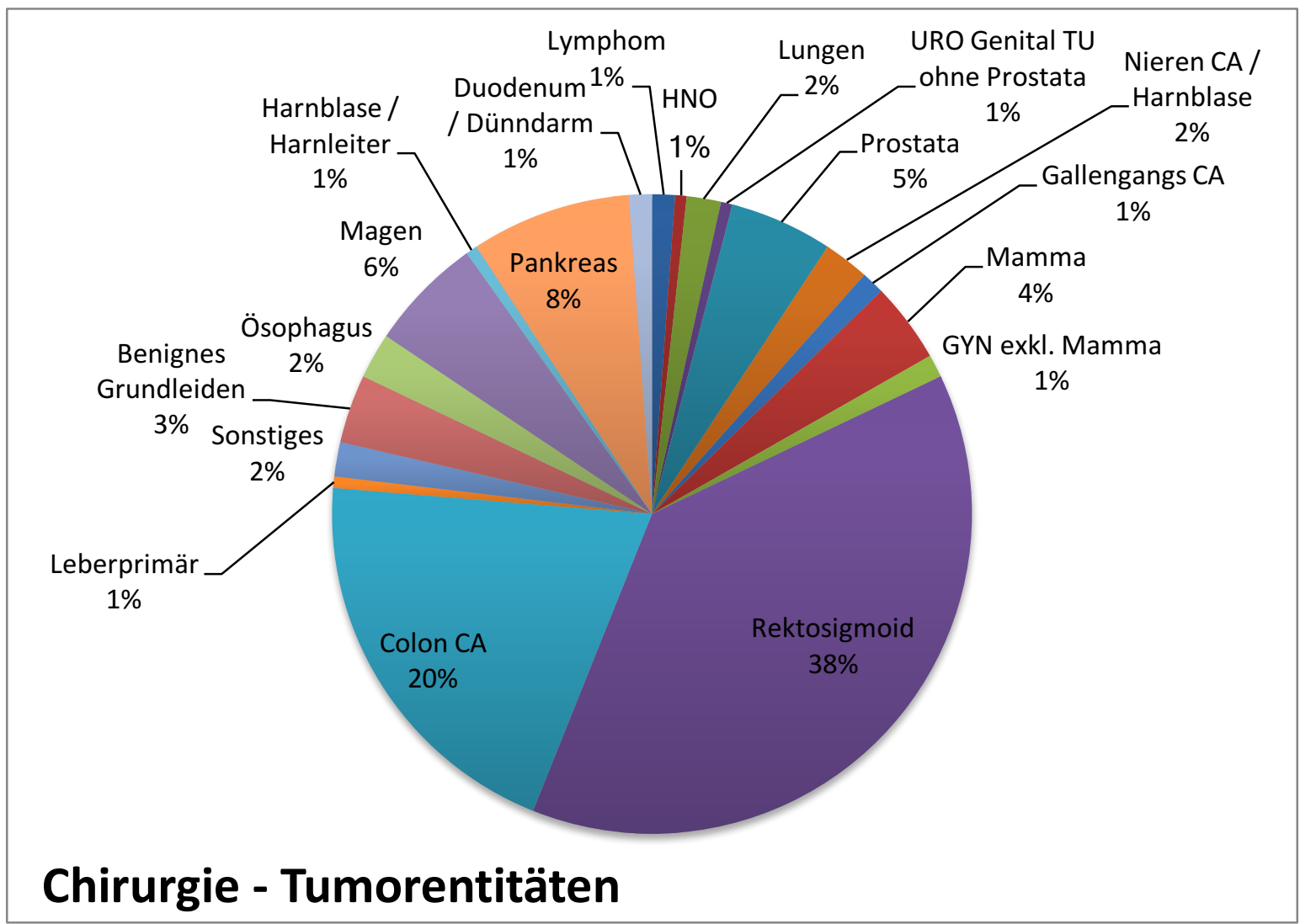

Abbildung 31: Chirurgie - Tumorentitäten.

Patienten mit der Tumorentität aus dem Gastrointestinaltrakt (Rektosigmoid, Colon- und Magen-CA) machen in der Chirurgischen Klinik zusammen 64,2 \% aus. Als weitere häufige Tumorentität ist das Pankreasmalignom mit 8 \% vertreten.

Der hohe Anteil der Tumorentität aus dem Magen-Darmtrakt resultiert aus dem hohen Anteil von Männern in der chirurgischen Patientengruppe (65\% Männer in der chirurgischen Patientengruppe und $48 \%$ in der radiologischen Patientengruppe). Siehe hierzu den oben stehenden Auszug aus dem hessischen Krebsregister 2008 (Abb.: 29, S.35) [11].

Die Patienten zur Portanlage in der Chirurgischen Klinik werden fast ausschließlich aus dem eigenen Patientenkollektiv akquiriert. 
3.2.2 TUMORENTITÄTEN UND HÄUFIGKEITEN IN DER RADIOLOGIE

Häufigkeiten nach Grundleiden der Erkrankung in der Radiologie:

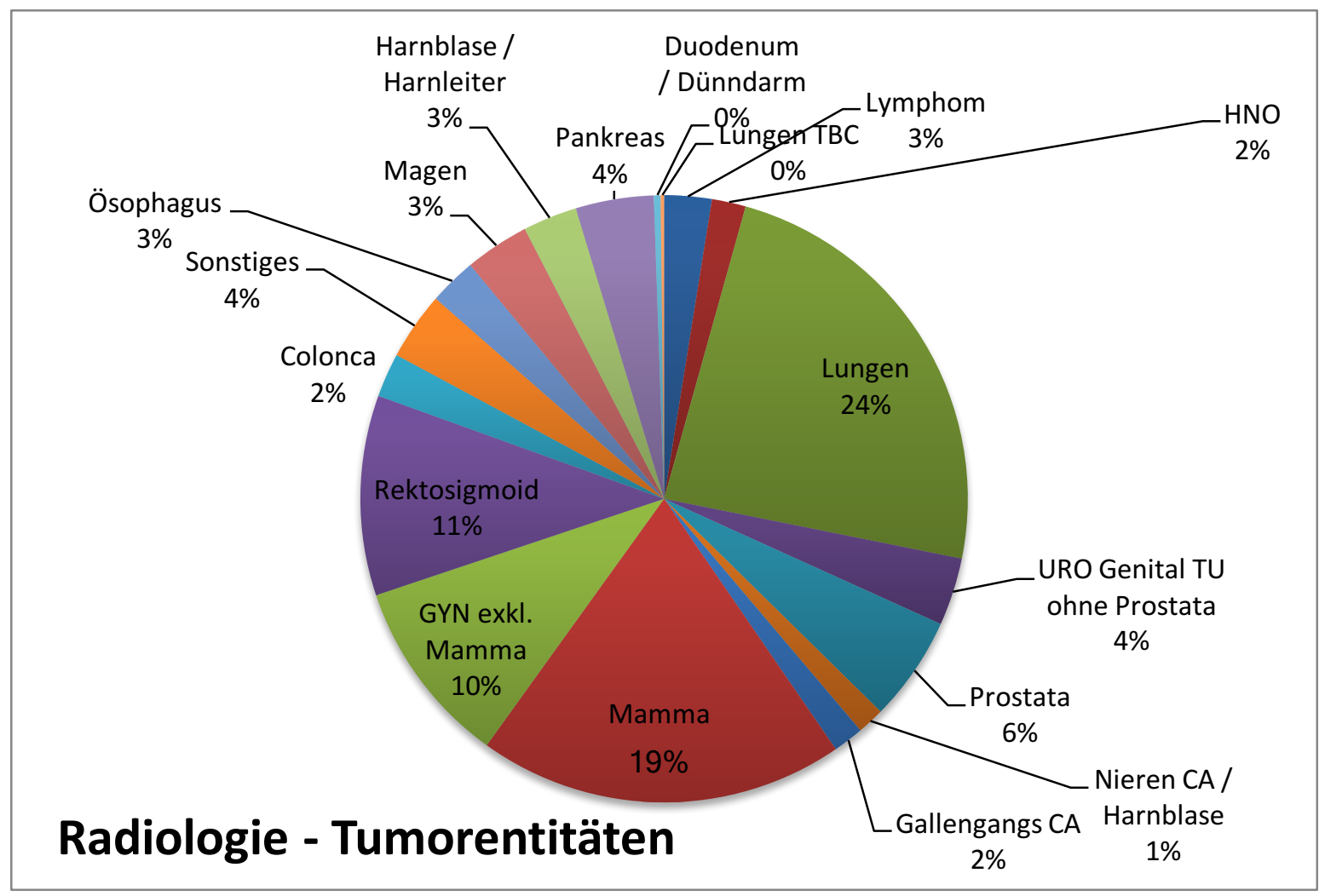

Abbildung 32: Radiologie - Tumorentität.

Die Verteilung der Tumorentitäten im Radiologischen Institut ist, wie bereits erwähnt, wesentlich heterogener. Den Hauptanteil stellen

- Lungentumore $(23,8 \%)$,

- Mammatumore (19,5\%) und

- Rektosigmoidale Tumore (10,6 \%) dar.

Zusammen ergeben die drei Tumorentitäten einen Anteil von 53,9 \%. 


\begin{tabular}{|c|c|c|c|c|}
\hline \multicolumn{3}{|l|}{ Abteilung } & \multirow{2}{*}{\begin{tabular}{|l} 
Häufigkeit \\
80
\end{tabular}} & \multirow{2}{*}{\begin{tabular}{|l} 
Prozent \\
46,2
\end{tabular}} \\
\hline Chirurgie & Gültig & nein & & \\
\hline & & ja & 93 & 53,8 \\
\hline & & Gesamt & 173 & 100,0 \\
\hline \multirow[t]{3}{*}{ Radiologie } & \multirow[t]{3}{*}{ Gültig } & nein & 228 & 41,2 \\
\hline & & ja & 326 & 58,8 \\
\hline & & Gesamt & 554 & 100,0 \\
\hline
\end{tabular}

Tabelle 4: Anteil der Patienten mit Metastasierung bei Portimplantation nach Abteilung.

Ein signifikanter Unterschied zwischen dem Anteil der Patienten mit Metastasen ergibt sich zwischen den Abteilungen nicht $(p=0,237)$. Hieraus kann vorsichtig auf eine ähnliche Morbidität geschlossen werden. 


\begin{tabular}{|c|c|c|c|c|c|c|}
\hline \multicolumn{7}{|c|}{ Facharzt vs. Assistenzarzt } \\
\hline \multicolumn{3}{|l|}{ Abteilung } & \multirow{2}{*}{\begin{tabular}{|l} 
Häufigkeit \\
121
\end{tabular}} & \multirow{2}{*}{\begin{tabular}{|l} 
Prozent \\
69,9
\end{tabular}} & \multirow{2}{*}{$\begin{array}{l}\text { Gültige } \\
\text { Prozente }\end{array}$} & \multirow{2}{*}{\begin{tabular}{|l} 
Kumulierte \\
Prozente
\end{tabular}} \\
\hline Chirurgie & Gültig & Facharzt & & & & \\
\hline & & Assistenzarzt & 52 & 30,1 & 30,1 & 100,0 \\
\hline & & Gesamt & 173 & 100,0 & 100,0 & \\
\hline \multirow[t]{5}{*}{ Radiologie } & \multirow[t]{3}{*}{ Gültig } & Facharzt & 469 & 84,7 & 84,8 & 84,8 \\
\hline & & Assistenzarzt & 84 & 15,2 & 15,2 & 100,0 \\
\hline & & Gesamt & 553 & 99,8 & 100,0 & \\
\hline & Fehlend & & 1 & 0,2 & & \\
\hline & \multicolumn{2}{|l|}{ Gesamt } & 554 & 100,0 & & \\
\hline
\end{tabular}

Tabelle 5: Portimplantationen durchgeführt von Facharzt oder Assistenzarzt. 


\begin{tabular}{|c|c|c|c|c|c|c|}
\hline \multicolumn{7}{|c|}{ Porthersteller } \\
\hline \multicolumn{3}{|l|}{ Abteilung } & \multirow{2}{*}{\begin{tabular}{|l} 
Häufigkeit \\
173
\end{tabular}} & \multirow{2}{*}{\begin{tabular}{|l|} 
Prozent \\
100,0
\end{tabular}} & \multirow[t]{2}{*}{\begin{tabular}{|l} 
Gültige \\
Prozente
\end{tabular}} & \multirow[t]{2}{*}{$\begin{array}{l}\text { Kumulierte } \\
\text { Prozente }\end{array}$} \\
\hline Chirurgie & & $\begin{array}{l}\text { Biomedica } \\
\text { Z-Port } \\
\text { (F8) }\end{array}$ & & & & \\
\hline \multirow[t]{11}{*}{ Radiologie } & \multirow[t]{9}{*}{ Gültig } & $\begin{array}{l}\text { Biomedica } \\
\text { Z-Port } \\
\text { (F8) }\end{array}$ & 361 & 65,2 & 65,3 & 65,3 \\
\hline & & $\begin{array}{l}\text { Zambon } \\
\text { F8 }\end{array}$ & 139 & 25,1 & 25,1 & 90,4 \\
\hline & & PFM F6 & 24 & 4,3 & 4,3 & 94,8 \\
\hline & & PFM F8 & 7 & 1,3 & 1,3 & 96,0 \\
\hline & & BARD F8 & 10 & 1,8 & 1,8 & 97,8 \\
\hline & & Dispomed & 2 & 0,4 & 0,4 & 98,2 \\
\hline & & $\begin{array}{l}\text { Basic- } \\
\text { Vaxel }\end{array}$ & 8 & 1,4 & 1,4 & 99,6 \\
\hline & & $\begin{array}{l}\text { Lifecath- } \\
\text { Picc Line } \\
\text { Katheter }\end{array}$ & 2 & 0,4 & 0,4 & 100,0 \\
\hline & & Gesamt & 553 & 99,8 & 100,0 & \\
\hline & Fehlend & & 1 & 0,2 & & \\
\hline & \multicolumn{2}{|l|}{ Gesamt } & 554 & 100,0 & & \\
\hline
\end{tabular}

Tabelle 6: Verwendete Porthersteller. 
In der Chirurgischen Klinik wurden ausschließlich Metall-Ports der Firma Biomedica, ZPorts implantiert. Im Radiologischen Institut hingegen wurden Ports verschiedener Hersteller verwendet.

In Arbeiten von Kock et al. sowie Wenke et al. wurden unterschiedliche Portkathetersysteme eingesetzt, ohne einen signifikanten Unterschied in der Komplikationsrate festzustellen [15; 31]. In Konsequenz daraus sollten die Komplikationsraten von den verwendeten Portsystemen unabhängig sein. 


\begin{tabular}{|c|c|c|c|c|c|}
\hline \multicolumn{6}{|c|}{ Klinisch apparente Komplikationen innerhalb von 30 Tagen } \\
\hline \multicolumn{3}{|l|}{ Abteilung } & \multirow{2}{*}{$\frac{\text { Häufigkeit }}{163}$} & \multirow{2}{*}{\begin{tabular}{|l} 
Prozent \\
94,2
\end{tabular}} & \multirow{2}{*}{$\begin{array}{l}\text { Gültige } \\
94,2\end{array}$} \\
\hline Chirurgie & Gültig & keine & & & \\
\hline & & Thrombose V. subclavia / Cava & 4 & 2,3 & 2,3 \\
\hline & & Infekt, Sepsis & 4 & 2,3 & 2,3 \\
\hline & & Mechanische Probleme & 2 & 1,2 & 1,2 \\
\hline & & Gesamt & 173 & 100,0 & 100,0 \\
\hline \multirow[t]{8}{*}{ Radiologie } & \multirow[t]{8}{*}{ Gültig } & keine & 535 & 96,6 & 96,6 \\
\hline & & Thrombose V. subclavia / Cava & 5 & 0,9 & 0,9 \\
\hline & & $\begin{array}{l}\text { Verschluss I Encasement } \\
\text { Portschlauch }\end{array}$ & 3 & 0,5 & 0,5 \\
\hline & & Infekt, Sepsis & 2 & 0,4 & 0,4 \\
\hline & & Mechanische Probleme & 1 & 0,2 & 0,2 \\
\hline & & Sonstige & 3 & 0,5 & 0,5 \\
\hline & & $\begin{array}{l}\text { Pneumothorax } \\
\text { /Spitzenpneumothorax }\end{array}$ & 5 & 0,9 & 0,9 \\
\hline & & Gesamt & 554 & 100,0 & 100,0 \\
\hline
\end{tabular}

Tabelle 7: Klinisch apparente Komplikationen innerhalb von 30 Tagen. 
Bei den Frühkomplikationen ergibt sich mit einem $p$-Wert von 0,022 ein signifikanter Unterschied zu Gunsten des Radiologischen Instituts.

Verfahrensbedingt existiert das Risiko eines Pneumothoracis bei der chirurgischen Implantationstechnik ohne Direktpunktion der V. subclavia nicht. 
3.4.2 SPÄTKOMPLIKATIONEN

Klinisch apparente Komplikationen nach 30 Tagen

\begin{tabular}{|c|c|c|c|}
\hline \multicolumn{2}{|l|}{ Abteilung } & \multirow{2}{*}{$\begin{array}{l}\text { Häufigkeit } \\
157\end{array}$} & \multirow{2}{*}{$\begin{array}{l}\text { Prozent } \\
90,8\end{array}$} \\
\hline Chirurgie & keine & & \\
\hline & Thrombose V. subclavia /Cava & 4 & 2,3 \\
\hline & $\begin{array}{l}\text { Verschluss / Encasement } \\
\text { Portschlauch }\end{array}$ & 1 & 0,6 \\
\hline & Infekt, Sepsis & 7 & 4,0 \\
\hline & Mechanische Probleme & 1 & 0,6 \\
\hline & Chemo nicht toleriert & 3 & 1,7 \\
\hline & Gesamt & 173 & 100,0 \\
\hline \multirow[t]{6}{*}{ Radiologie } & keine & 518 & 93,5 \\
\hline & Thrombose V. subclavia /Cava & 6 & 1,1 \\
\hline & $\begin{array}{l}\text { Verschluss/Encasement } \\
\text { Portschlauch }\end{array}$ & 11 & 2,0 \\
\hline & Infekt, Sepsis & 16 & 2,9 \\
\hline & Mechanische Probleme & 3 & 0,5 \\
\hline & Gesamt & 554 & 100,0 \\
\hline
\end{tabular}

Tabelle 8: Klinisch apparente Komplikationen nach 30 Tagen. 
Bei den klinisch apparenten Spätkomplikationen ergibt sich mit einem $p$-Wert von 0,021 ein signifikanter Unterschied zu Gunsten der radiologischen Implantation.

3.4.3 RADIOLOGISCHE FRÜHKOMPLIKATION PNEUMOTHORAX FACHARZT VS. ASSISTENZARZT

\begin{tabular}{|c|c|c|c|c|}
\hline \multicolumn{5}{|c|}{ Komplikation Pneumothorax innerhalb 30 Tage * Facharzt vs. Assistenzarzt } \\
\hline & & Facharzt & Assistenzarzt & \\
\hline Kein Pneumothorax & Anzahl & 464 & 84 & 548 \\
\hline \multirow[t]{2}{*}{ Pneumothorax } & Anzahl & 5 & 0 & 5 \\
\hline & Prozent & $1,1 \%$ & $0,0 \%$ & $0,9 \%$ \\
\hline |Gesamt & & 469 & 84 & 553 \\
\hline
\end{tabular}

Tabelle 9: Frühkomplikation Pneumothorax Radiologie.

Die von einem Facharzt ausgeführten Punktionen führten bei Verwendung einer $18 \mathrm{G}$ Nadel bei insgesamt 5 Fällen von insgesamt 464 Portimplantationen zu einem Pneumothorax (1,1 \%). Die von einem Assistenzarzt ausgeführten Punktionen mittels einer 21 G Nadel führten in keinem Fall der insgesamt 84 Portimplantationen zu einem Pneumothorax (0\%).

Trotz dieser Werte ergibt sich mit einem $p$-Wert von 0,342 kein signifikanter Wert. 


\section{DISKUSSION}

\subsection{IMPLANTATIONSVERFAHREN CHIRURGISCHER UND RADIO- LOGISCHER PORTSYSTEME}

Ziel dieser Arbeit war es, in einer retrospektiven Studie nach klinisch apparenten Frühund Spätkomplikationen bei chirurgischen und radiologischen Portimplantationen zu suchen. Über einen Zeitraum von zwei Jahren wurden alle Portimplantationen der Chirurgischen Klinik und des Radiologischen Instituts ausgewertet. Wir entschieden uns für einen Zeitraum, in dem auch ein radiologischer Assistenzarzt mit einer neuen, im Durchmesser geringeren Punktionsnadel angelernt wurde, um die Sicherheit der Punktion gegen einen erfahreneren Facharzt bezüglich des Risikos des Pneumothoracis aufzuzeigen.

Die Gemeinsamkeiten der chirurgischen und radiologischen Portimplantation in unserer Klinik bestehen in der Zugangsvene (V. subclavia / V. cephalica) und der pektoralen Lage, die Unterschiede sind die Seitenbevorzugung (chirurgisch rechts pektorale Implantation, radiologisch links pektoral) sowie die Implantationstechnik Cutdown auf die Vene (chirurgisch) und die Punktion unter Durchleuchtungskontrolle (radiologisch) nach vorangehender Phlebographie.

4.1.1 IMPLANTATIONSBEDINGTE VORTEILE DER CHIRURGISCHEN VS. RADIOLOGISCHEN IMPLANTATION

Durch die chirurgische Implantationstechnik mittels Cutdown sind die periprozeduralen Vorteile der erfolgreichen chirurgischen Portimplantation, wie unter 2.8.2 erwähnt:

A) Fehlen des Pinch-off-Syndroms.

B) Fehlen des Pneumothoracis.

Ein Pinch-off-Syndrom war im radiologischen Kollektiv (bei bildgesteuerter Punktion in Roadmaptechnik im mittleren / lateralen Drittel der V. subclavia) jedoch ebenfalls nicht zu beobachten. 
Wie in Kapitel 2.9.2 beschrieben, bringt die radiologische, durchleuchtungskontrollierte Portimplantation nach einer vorausgehenden Venographie den Vorteil mit sich, dass Normvarianten und Komplikationen erkannt werden können und ggf. eine Adaption der Implantationsmethode erfolgen kann (siehe Abb. 27 und 28).

\subsubsection{PUNKTION DER V. JUGULARIS VS. V. SUBCLAVIA}

Im radiologischen Patientenkollektiv erfolgte die Portimplantation nach Phlebographie, bildgesteuert über die Punktion der V. subclavia.

Die bildgesteuerte Punktion unter Ultraschall oder Venographie führt zur deutlichen Reduktion perioperativer Komplikationen [24] und sollte als Standardmethode verwendet werden. Ohne Ultraschallkontrolle stellt die arterielle Fehlpunktion der A. subclavia oder $\operatorname{der}$ A. carotis communis eine der häufigsten periprozeduralen Komplikationen dar [6; 25].

Laméris et al. weisen bei bildgesteuerter Punktion der V. subclavia einen Rückgang der Punktionskomplikationen gegenüber Blindpunktionen von $10 \%$ auf $0 \%$ nach [16].

In einer Studie von Teichgräber et al. wird die Punktion der V. juglaris interna bevorzugt, da diese gegenüber der $\mathrm{V}$. subclavia besser ultraschallgestützt zu punktieren sei [26]. Zähringer et al. geben in Bezug auf die ultraschallgestützte Punktion der V. subclavia eine technische Erfolgsrate von $100 \%$ bei 271 Portimplantationen an. Es kam zu keinen relevanten periprozeduralen Komplikationen (kein Pneumothorax, keine arterielle Fehlpunktion) [34].

In unserem Patientenkollektiv konnte ebenfalls unter Roadmaptechnik nach Phlebographie bei allen Patienten ein Subklaviaport implantiert werden.

Wagner et al. beschreiben in einer prospektiven Studie mit 50 Patienten bei ultraschallgesteuerter Punktion der V. jugularis interna keine Akzeptanzprobleme bezüglich des kosmetischen Resultats [29]. Hingegen berichten Zähringer et al. bei regelhafter Demarkierung des subkutanen Katheterverlaufs über die Klavikula über Akzeptanzprobleme bei Patienten und Zuweisern und wechselt auf die Implantation über die V. subclavia [34]. Die Hauptindikation für den Zugang über die V. jugularis interna - die Vermeidung des Pinch-off-Syndroms - konnte in unserer Studie bei lateralem Zugang über die V. subclavia nicht beobachtet werden. 




Abbildung 33: Auswärts eingelegter Port über die V. jugularis interna. Der Portschlauch wird über die Klavikula getunnelt und demarkiert sich unter der Haut. 


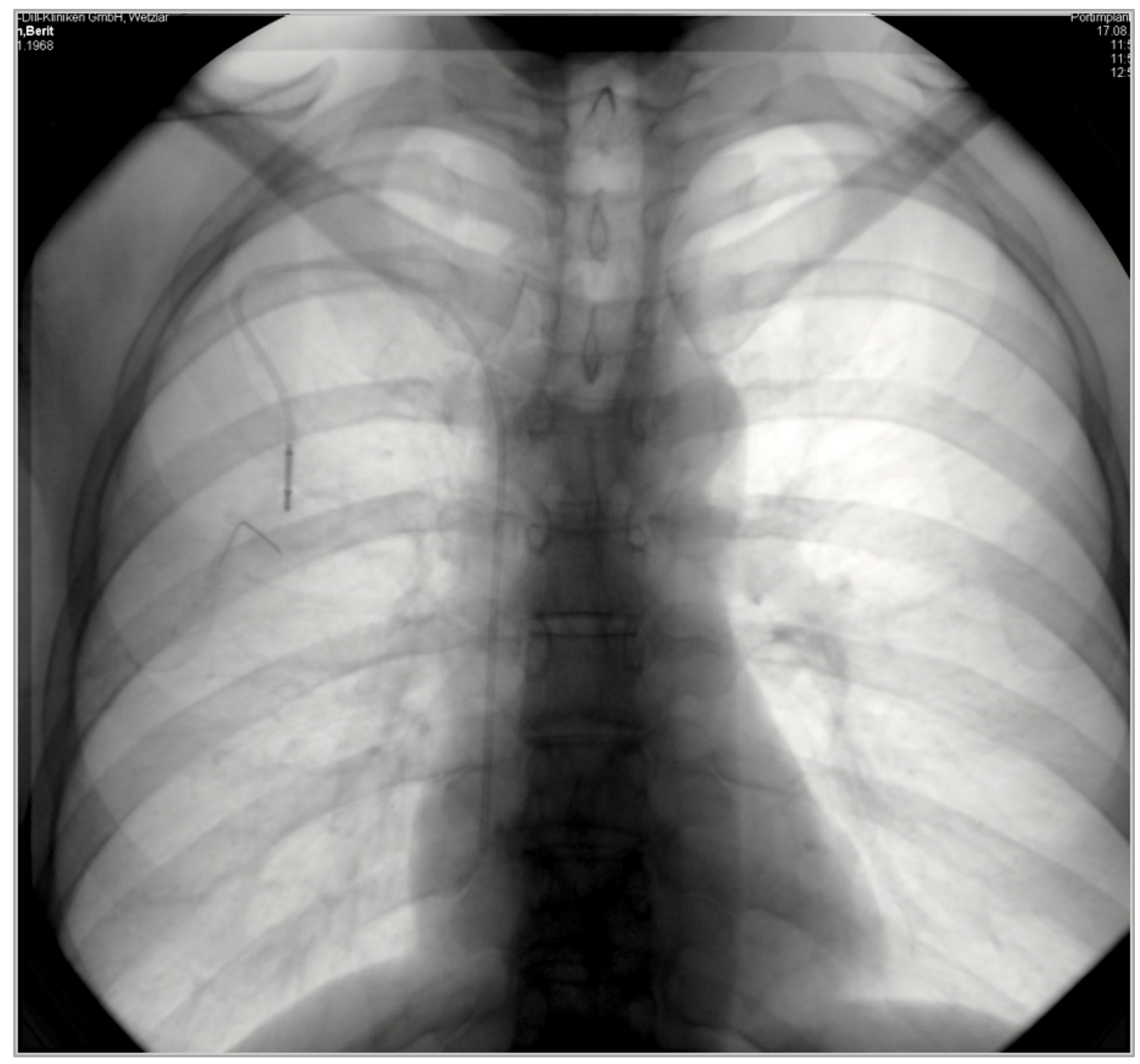

Abbildung 34: Gleiche Patientin wie in Abb. 32: Auf Wunsch der Patientin Entfernung des links seitigen Jugularis-Interna-Ports und Implantation über die V. subclavia rechts.

Eine Metaanalyse (mit 3420 Kathetern in zehn Studien) erbrachte keinen signifikanten Unterschied zwischen einer Punktion der V. jugularis interna und der V. subclavia bezüglich des Risikos eines Pneumo- oder Hämatothoracis. Die gleiche Metaanalyse zeigte bei einem Zugang über die V. jugularis interna (sechs Studien und 2020 Katheter) eine signifikant höhere Rate an arteriellen Punktionen verglichen mit Zugängen über die $\mathrm{V}$. subclavia (3.0\% vs. $0.5 \%)$ [22]. 
Mit einem $p$-Wert von 0,022 ergab sich bei den Frühkomplikationen ein signifikanter Wert zu Gunsten des radiologischen Implantationsverfahrens.

\section{Fühkomplikationen Chirurgie $(n=173)$}

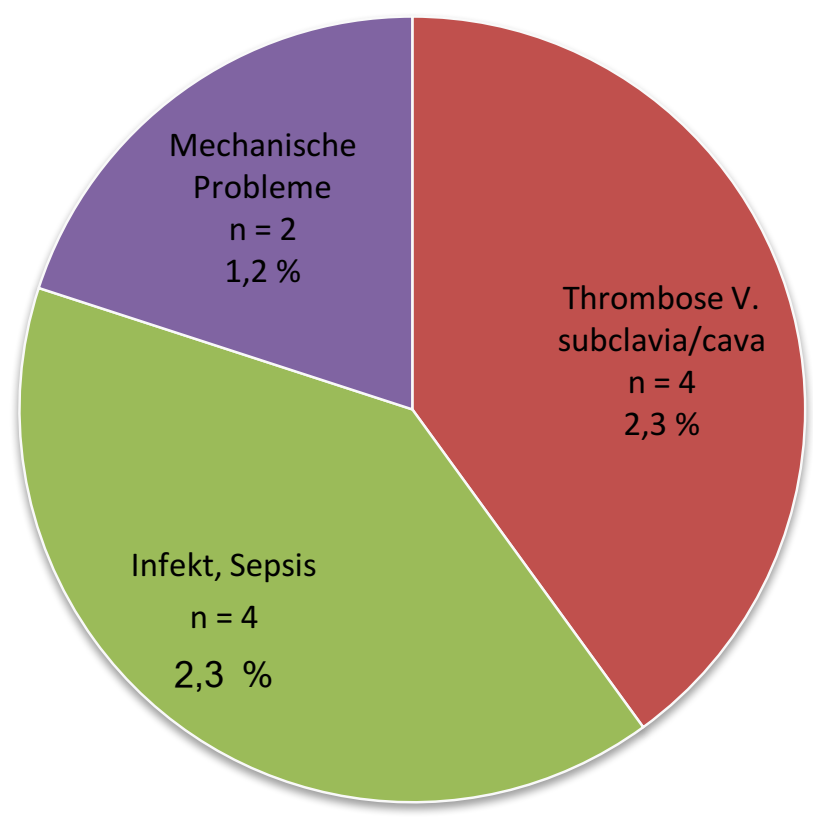

Abbildung 35: Frühkomplikationen in der Chirurgie.

Die Thrombose der V. subclavia und der V. Cava superior kommen im chirurgischen Patientenkollektiv mit 2,3 \% genauso häufig vor wie die Frühkomplikation des Portinfekts / der Sepsis. Mechanische Probleme treten erst an Platz 3 mit einer Häufigkeit von 1,2 \% auf. 


\section{Frühkomplikation Radiologie $(n=554)$}

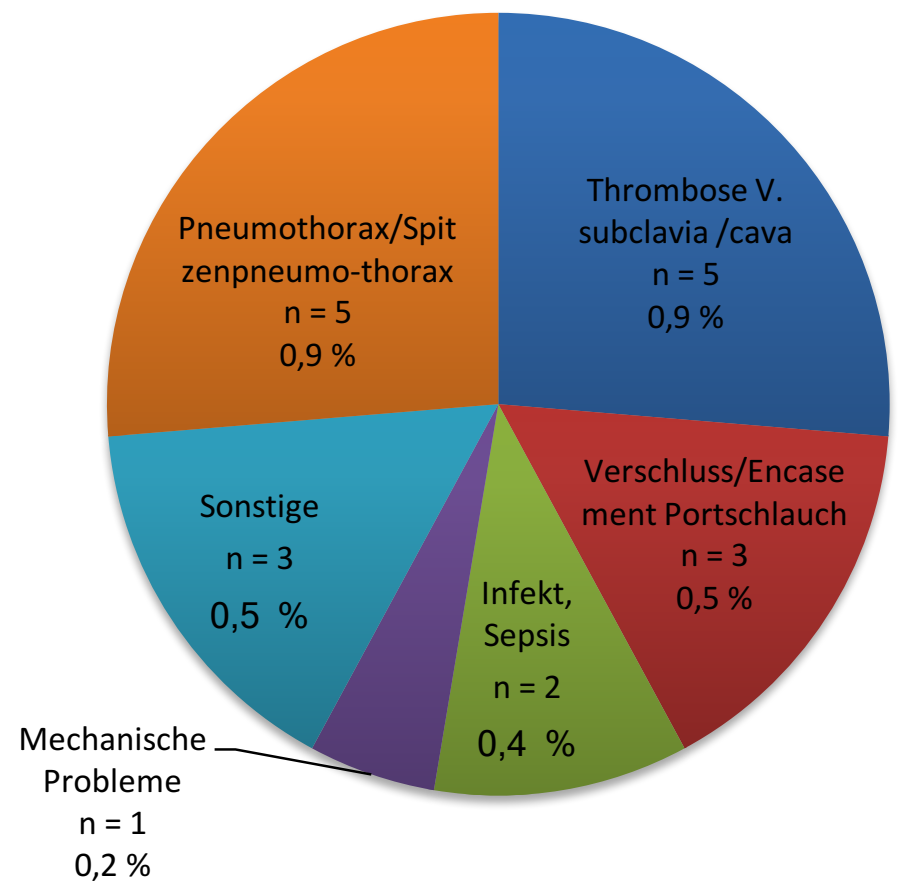

Abbildung 36: Frühkomplikationen in der Radiologie.

Bei den radiologischen Frühkomplikationen stellen der Pneumothorax und die Thrombose der V. subclavia / V. cava superior mit einer Häufigkeit von $0,9 \%$ die beiden Hauptkomplikationen dar. Infekt / Sepsis und Portschlauchverschluss / Encasement stellen mit mechanischen- und sonstigen Problemen jeweils Frühkomplikationen unter 1 \% dar.

Die Gesamtzahl der implantierten Ports in der Chirurgischen Klinik betrug 173 vs. 554 Portimplantationen im Radiologischen Institut. Bei den Frühkomplikationen ( $<30 \mathrm{~d}$ ) ergibt sich mit einem $p$-Wert von 0,022 ein signifikanter Wert zu Gunsten der radiologischen Portimplantation. Bezüglich aller Frühkomplikationen (Infekt / Sepsis, Venenthrombose / Portschlauchverschluss und mechanischer Probleme) schneidet das radiologische Verfahren signifikant besser ab. 


\section{Relative Häufigkeiten Frühkomplikationen Chirurgie}

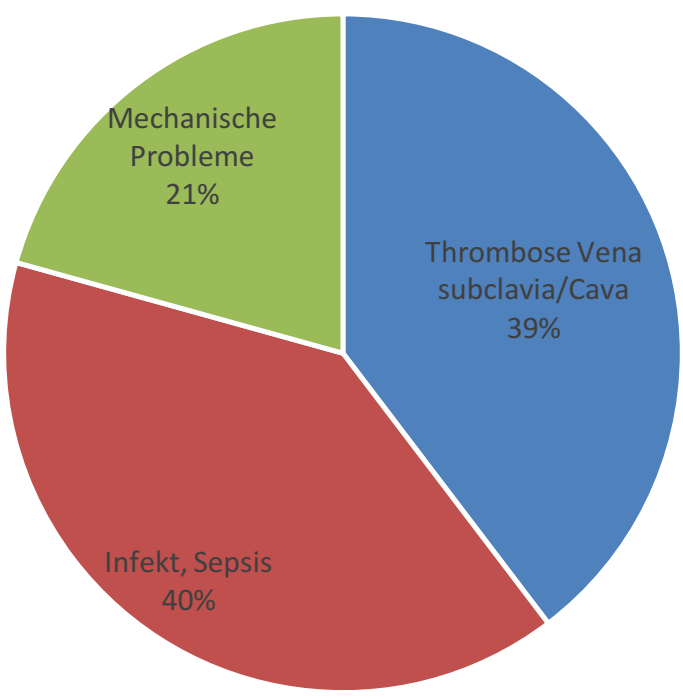

Abbildung 37: Relative Häufigkeiten Frühkomplikationen in der Chirurgie.

\section{Relative Häufigkeiten Frühkomplikationen Radiologie}

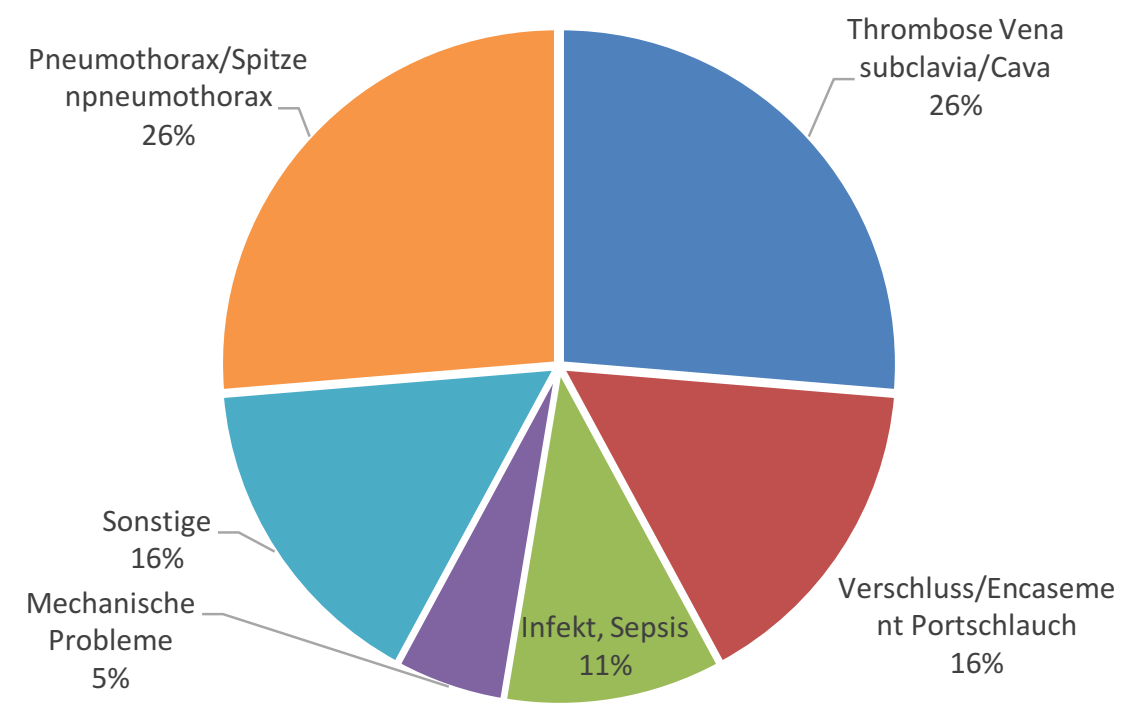

Abbildung 38: Relative Häufigkeiten Frühkomplikationen in der Radiologie. 
4.2.1 PNEUMOTHORAX

Die periprozedurale Komplikation des Pneumothoracis ist verfahrensbedingt durch die Punktion der V. subclavia bei der radiologischen Implantation möglich, in der chirurgischen Implantation hingegen aufgrund des Implantationsverfahrens (venöser Cutdown) nicht existent.

Im radiologischen Patientengut traten bei 554 durchgeführten Portimplantationen über die V. subclavia 5 Pneumothoraces auf, dies entspricht 0,9\%. Verglichen mit anderen Beschreibungen von Portkatheter Anlagen mit direkter Punktion der V. subclavia, die Prozentzahlen zwischen 3,2 \% [14], 5,6 \% [9] und 5,7 \% [10] angeben, liegt die Pneumothoraxrate in unserem Kollektiv damit deutlich niedriger.

Die Komplikationen des Pneumothoracis und des Pinch-off-Syndroms sind auch unter vielen Radiologen ein Hauptargument gegen die Implantation eines Portsystems mit Zugang über die V. subclavia, weshalb unter anderem die V. jugularis interna oder Unterarmvenen [2] bevorzugt werden [26; 29; 34].

In einer Metaanalyse mit 17 prospektiven Studien (2085 jugularis- und 2428 subclaviaKathetern) konnte gezeigt werden, dass es mehr arterielle Fehlpunktionen aber weniger Katheter-Fehllagen bei Zugängen über die V. jugularis interna gegenüber Zugängen via $V$. subclavia gab. Es gab keinen Beweis für einen Unterschied in der Inzidenz des Hämatooder Pneumothoracis und von Gefäßverschlüssen [22].

4.2.2 PNEUMOTHORAX ASSISTENZARZT (PUNKTIONSNADEL $21 \mathrm{G}$ ) VS. FACHARZT (PUNKTIONSNADEL $18 \mathrm{G}$ )

Die Portimplantationen in unserem Institut wurden von 2 Fachärzten mittels einer $18 \mathrm{G}$ Punktionsnadel und von einem interventionellen Anfänger mittels $21 \mathrm{G}$ Punktionsnadel durchgeführt. Bei 84 von einem Assistenzarzt mit einer Nadel mit geringerem Durchmesser (21 G) implantierten Portsystemen kam es zu keinem Pneumothorax. Von den zwei Fachärzten wurden insgesamt 496 Ports implantiert, wobei es bei fünf davon zu einem Pneumothorax kam, welcher jeweils keiner weiteren invasiven Therapie (Drainage) 
bedurfte.

Es ergab sich eine Komplikationsrate Assistenzarzt (21 G-Punktionsnadel) vs. Facharzt (18 G-Punktionsnadel) $0 \%$ zu 1,1 \%. Es lag jedoch kein signifikanter Unterschied zu Gunsten des geringeren Durchmessers vor $(p$-Wert $=0,342)$.

Die gering höhere Pneumothoraxrate der Fachärzte kann zum einen an komplexschwierigeren Eingriffen liegen, die geringere Komplikationsrate des Assistenzarztes zum anderen an einfacheren Patientenanatomien und der geringeren Zahl der Eingriffe.

Eine prospektiv-randomisierte Studie wäre notwendig, um die Fragestellung des Pneumothoracis und lokaler Komplikationen in Folge der Gefäßpunktion (z.B. Thrombosen und Blutungen) in Abhängigkeit vom Punktionskanülendurchmesser abschließend zu überprüfen.

\subsubsection{PORTINFEKTION}

Postoperative Entzündungen und Abszedierungen des Portlagers sowie eine Sepsis nach Portverwendung sind mögliche Frühkomplikationen, die eine Portkatheterentfernung erzwingen können.

In der Chirurgischen Klinik lag der Anteil der Portinfektionen / Sepsen mit 2,3 \% signifikant höher als im Radiologischen Institut mit 0,4 \%.

Der relative Anteil der Portinfektionen in der Gruppe der Frühkomplikation stellt mit $40 \%$ die Hauptfrühkomplikation in der Chirurgischen Klinik dar. Im Radiologischen Institut steht die Portinfektion (mit einem relativen Anteil von $11 \%$ ) in der Gruppe der Frühkomplikation an Rang fünf. Obwohl der chirurgische Eingriff im OP-Saal stattfand und der radiologische in einem Angiographieraum ohne Vorraum, kam es bei den chirurgischen Portimplantationen häufiger zu einer frühen Portinfektion.

Im Radiologischen Institut fällt der relative Anteil der Portinfektionen - gemessen an den Gesamtfrühkomplikationen - mit $11 \%$ nicht als Hauptkomplikation auf, jedoch stellt die Portinfektion immer einen Explantationsgrund dar. Bei weiterer Notwendigkeit eines Ports kann dieser auf der kontralateralen Seite oder nach Ausheilung des Infektes ipsilateral 
implantiert werden.

In anderen Studien liegen die Portinfektionsraten bei 3,8 \% [10] und 5,2 \% [13].

4.2.4 VENENTHROMBOSE

Eine Thrombose kann in zwei Formen entstehen: als Stenose oder Verschluss der Zugangsvene durch das Trauma der Katheteranlage induziert oder als Katheterspitzenthrombus durch intravaskuläre Proteine und Zelldeposition [30]. Erwartungsgemäß sollte es bei der chirurgischen Implantation (Cutdown und Präparation der V. cephalica) eigentlich zu weniger Komplikationen dieser Art kommen, da es hierbei zu keinem Trauma der V. subclavia kommt.

Die Venenthrombose:

- Chirurgische Implantation: 2,3\%.

- Radiologische Implantation: 1,3\%.

Mit einem relativen Anteil von $39 \%$ ist die Zahl der Venenthrombosen bei den Frühkomplikationen des chirurgischen Verfahrens allerdings ähnlich hoch wie die der Portinfektionen, stellt jedoch primär keinen Explantationsgrund dar. Mit einem relativen Anteil von $26 \%$ ist der Anteil der Venenthrombosen im Radiologischen Institut neben dem Pneumothorax (ebenfalls mit relativen Anteil von $26 \%$ ) auf dem ersten Platz der Hauptfrühkomplikationen.

Bei den Frühkomplikationen scheint die Verlaufsstrecke des Portkatheters keinen negativen Einfluss auf die Entstehung einer Venenthrombose zu haben. Die Verlaufsstrecke der radiologisch implantierten Portkatheter ist über die linke V. subclavia im Vergleich zur Verlaufsstrecke der chirurgischen Portschläuche über die rechte V. subclavia unserer Erfahrung nach in der Regel um etwa $5 \mathrm{~cm}$ länger.

Die Diagnostik bei klinischem Verdacht auf eine Venenthrombose erfolgt über einen venösen Zugang am zur Portimplantation ipsilateralen Arm. Nach Kontrastmittel-Gabe wird der venöse Abstrom vom Oberarm bis in die obere Hohlvene dargestellt. Eine 
Venenthrombose an sich stellt keine Funktionsbeeinträchtigung des Portsystems dar. Der Port kann in der Regel weiterbenutzt werden, solange das Ende des Portschlauches nicht im Thrombus liegt. Sollte die Venenthrombose nicht auf nieder-molekulares Heparin ansprechen und klinisch progredient werden, kann eine Explantation des Portsystems erforderlich werden.

In einer prospektiven Studie wurden die Patienten innerhalb eines Monats nach Implantation (18 G-Punktionsnadel, bildgesteuert (Venographie oder Ultraschall), V. subclavia, 89 Implantationen rechts, 3 Implantationen links) per Ultraschall untersucht. Die Ports wurden nach Implantation und in Intervallen von 1 bis 4 Wochen mit Heparin gespült: Initialuntersuchung:

- Kein Thrombus: $n=25(27 \%)$.

- Thrombus ohne Funktionsbeeinträchtigung: $n=64(70 \%)$.

- Thrombus mit Funktionsbeeinträchtigung $n=3$ (3\%) [33].

Im radiologischen Patientenkollektiv erfolgte die Untersuchung hingegen nur bei klinisch funktionsbeeinträchtigten Ports.

\subsubsection{ENCASEMENT / FIBRINUMSCHEIDUNG}

Primär fallen Fibrinumscheidungen (Encasements) des distalen Portschlauches klinisch durch schwergängige oder unmögliche Infusionsgaben auf. Ebenfalls ist eine Blutaspiration nicht mehr oder nur noch erschwert möglich.

Zur Diagnostik führten wir unter Verwendung von Einmalhandschuhen und Hautdesinfektion nach der Punktion der Portkammer mittels Hubernadel eine Portographie mit Darstellung des gesamten Portsystems durch. Die Portographie beinhaltet die Abbildung des gesamten Portsystems von der Portkammer bis zur Schlauchspitze des Portschlauches in der V. cava superior. Mechanische Probleme können im gesamten Portkammersystem auftreten und sollten nicht übersehen werden. Problemareale befinden sich hauptsächlich in drei Lokalisationen:

1. Proximal: 
Leckage der Portkammer / Portmembran mit Kontrastmittel-Paravasat (Punktion mittels normaler Nadel).

Defekt des proximalen Portschlauches durch Fehlpunktionen oder gelöste Muffe bei mangelnder Fixierung.

Verkippter Port durch gelöste Fadenfixierung.

2. Mittlerer Anteil:

Im mittleren Schlauchverlauf ist auf ein Pinch-off-Syndrom zu achten (Einengung des Portschlauches in der kostoklavikulären Enge).

3. Distaler Anteil:

Encasement / Fibrinumscheidung des distalen Portschlauches. Hierbei ist bei Kontrastmittel-Applikation kein typisches Jet-Phänomen am distalen Ende des Portschlauches zu sehen. Durch die Umscheidung des Portschlauches läuft das Kontrastmittel an der Spitze des Portschlauches in die Umscheidung und anschließend am Portschlauch nach kranial und tritt erst am Ende des Encasements in die V. cava. Bei kleineren Portschlauch-Thrombosierungen erfolgt eine Umlenkung des über die Portkammer applizierten Kontrastmittels zur Seite.

Ein Verschluss des Portsystems kann im gesamten Verlauf auftreten. Meist liegt hier eine fehlende oder nicht ausreichende Spülung des Systems nach Gebrauch zugrunde.

Im Falle einer venösen Abflussproblematik (Portschlauch- oder Portkammerverschluss) kann mittels einer $10 \mathrm{ml}$ Spritze $(\mathrm{NaCl})$ durch ein repetitives Anspülen und Aspirieren versucht werden, das Portsystem frei zu spülen. Bei erfolgreichem Manöver traten bei den Patienten keine Komplikationen im Sinne einer Dyspnoe oder Lungenembolie auf.

Ein Encasement wurde in der Chirurgischen Klinik als Frühkomplikation nicht auffällig, im Radiologischen Institut wurden insgesamt 3 Fälle beobachtet $(0,5 \%)$.

In einer Studie zu venographisch kontrollierten Portsystemen wurden in $78-87 \%$ der Portschläuche Thrombin-Umscheidungen gefunden [30]. Somit ist von einer höheren Rate von nicht klinisch auffälligen Portschlauch-Encasements auszugehen.

Zur Therapie eines durch Encasements nicht funktionstüchtigen Portsystems verwendeten 
wir eine Urokinase-Lyse von $250000 \mathrm{IE}$, aufgelöst in $50 \mathrm{ml} \mathrm{NaCl}$, die über einen Perfusor, welcher an die Portkammer angeschlossen wurde, in 4 - 5 Stunden einlief (50 000 IE / h). Nach der Lyse erfolgte die Portographie. Bei komplett aufgelöstem Encasement sind eine Aspiration und ein Spülen ohne großen Druckaufwand möglich. Bei noch bestehendem Thrombus wird eine Wiederholung der gleichen Prozedur durchgeführt. Bei ausbleibendem Erfolg muss eine Portexplantation in Erwägung gezogen werden.

In einer Studie (ebenfalls mit 250000 IE Urokinase (30 000 IE / h) konnte eine Wiedererlangung der Funktion in $97 \%$ der Fälle nachgewiesen werden [32].

In einer anderen Studie wurde eine Alteplase-Lyse durchgeführt und eine Wiedereröffnungsrate von 87 \% bei einer Gabe von ein bis zwei Mal je 2 mg rtPA mit einer maximalen Einwirkzeit von 120 min nachgewiesen. Zu intrakraniellen Hämorrhagien kam es hierbei nicht [4]. Dagegen geben Whigham et al. eine Erfolgsrate von 92,9 \% bei einer Dosis von 1 - 4 mg rtPA an [32].

Eine andere Therapiemethode ist das perkutane Stripping des Thrombus, welches eine technische Erfolgsrate von 89 \% aufweist [32], jedoch im Vergleich zur Lyse invasiv ist und als Komplikationsmöglichkeit eine potentielle Pulmonalarterien-Emboliserung aufweist.

In einer prospektiv-randomisierten Studie zum Vergleich von perkutan-venösem Stripping und Urokinaselyse (250000 IE, 30000 IE / h) konnte kein signifikanter Unterschied gefunden werden [7].

Aufgrund des langen Zeitaufwandes der Urokinaselyse von mindestens $4 \mathrm{~h}$ sind wir aktuell zur Lyse mittels Alteplase gewechselt. Rathmann et al. berichten von einer Erfolgsrate von $52 \%$ nach $2 \mathrm{mg}$ Alteplase über $30 \mathrm{~min}$ und von einer Erfolgsquote von $84 \%$ nach Wiederholung der Prozedur [20]. 


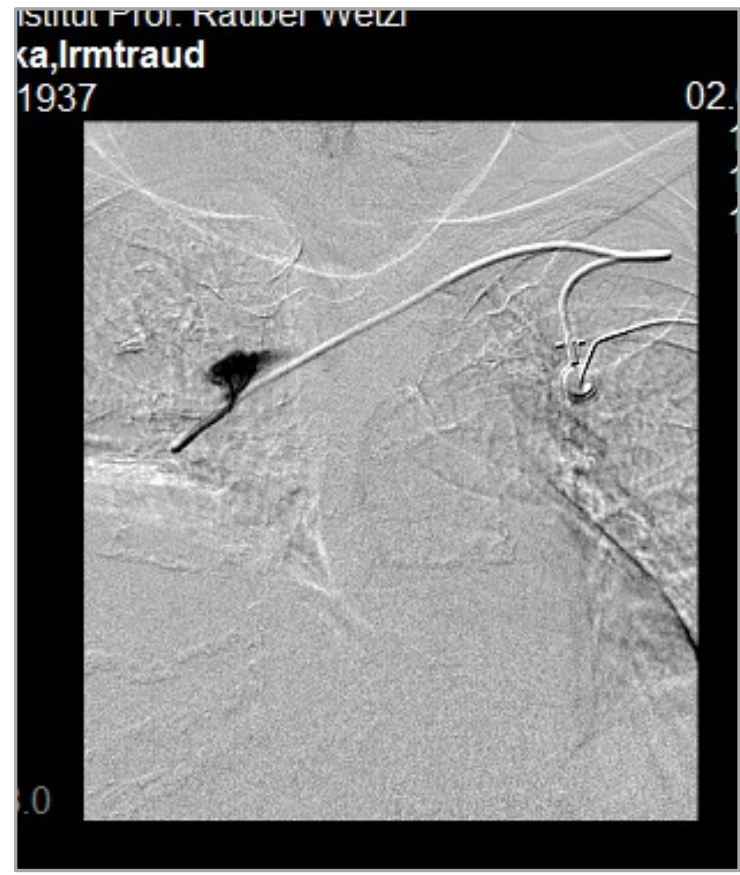

Abbildung 39: Das KM läuft entlang des Portschlauches nach kranial aufgrund der Fibrin-Umscheidung des Portschlauches und tritt am Ende der Fibrin-Umscheidung in die $V$. cava superior über. Häufiger Nebenbefund beim Encasement ist, wie hier auch, ein nach kranial dislozierter Portschlauch.

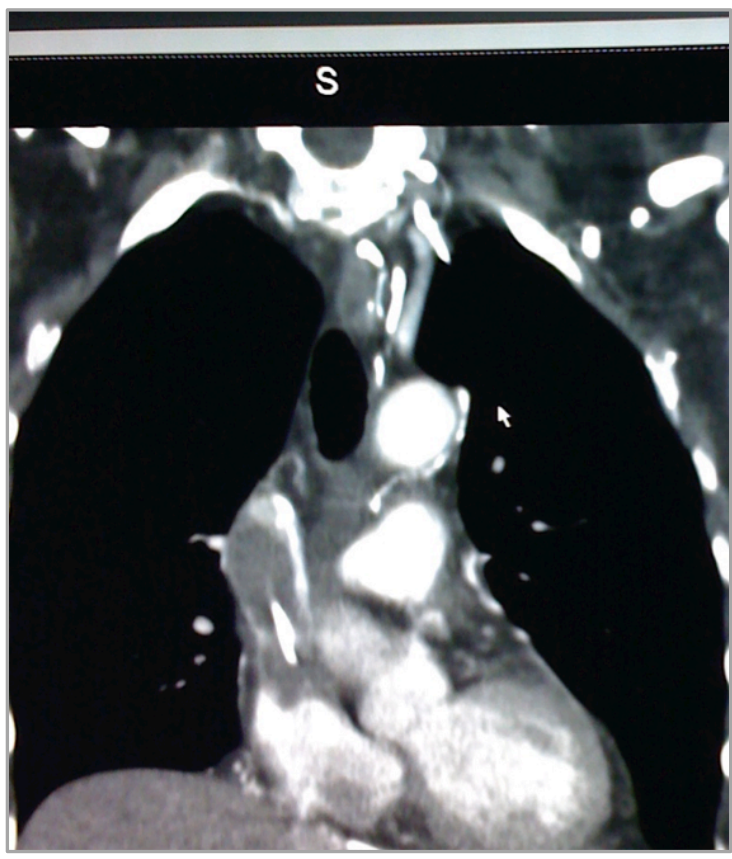

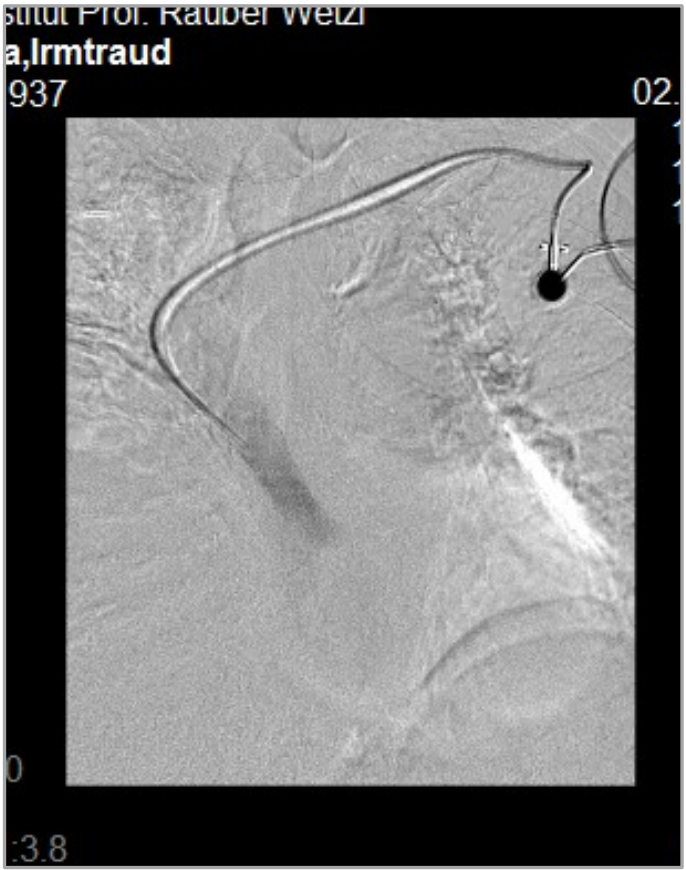

Abbildung 40: Gleiche Patientin wie in Abb. 39, nach erfolgreicher Lyse. Unauffälliger KMAbstrom, sowie spontan nach kaudal repositionierter Portschlauch.
Abbildung 41: Anderer Pat. mit ausgeprägtem Encasement. Um den Portschlauch ausgeprägter Thrombus in koronarer Rekonstruktion. 
4.2.6 MECHANISCHE PROBLEME

Die mechanische Frühkomplikation ist mit einem relativen Anteil von $21 \%$ bei der chirurgischen Implantation die dritthäufigste Hauptfrühkomplikation, bei der radiologischen Implantation mit einem Anteil von $5 \%$ an den Frühkomplikationen an Position sechs.

- Mechanische Probleme: - Chirurgie 1,2\%.

- Radiologie 0,2\%.

- Mechanische Frühkomplikationen in anderer Arbeiten: 3,3 \% [30].

Unter mechanischen Problemen subsummieren sich Defekte der Portmembran und des proximalen Portschlauches. Diese sind auf eine unsachgemäße Punktion zurückzuführen. Obsolet ist eine Portmembranpunktion mit einer normalen Nadel, da diese die Portmembran stanzt.

Verkippte Portkammern aufgrund mangelnder Fixation oder aus der Pectoralisfaszie gelösten Fäden gehören ebenfalls zu den mechanischen Problemen. In einer anderen Arbeit wurde auf eine Fixierung der Portkammer auf der Pectoralisfaszie verzichtet und keine signifikant häufigere Dislokation festgestellt [10]. Bei verkippten oder gedrehten Ports ist die Portmembran auch unter Durchleuchtung selten oder gar nicht punktabel. Damit machen diese mechanischen Komplikationen regelhaft eine Portexplantation und eine Port-Neuimplantation notwendig. In diesen Fällen wird das alte Portsystem komplett gegen ein neues Portsystem ausgetauscht - möglichst ohne eine erneute Punktion durchzuführen. Hierzu wird der alte Portschlauch über einen Führungsdraht ausgetauscht.

Ein weiteres mechanisches Problem kann ein Umschlagen des Portschlauches aus der V. cava superior in die V. jugularis sein. In diesem Falle ist über einen venösen Zugang der Leiste (V. femoralis) eine Reposition mittels Katheter oder einer Schlinge möglich. 
4.2.7 PINCH-OFF-SYNDROM (POS)

Ein Pinch-off-Syndrom kann ebenfalls als mechanisches Problem auftreten. Wie bereits oben beschrieben, kommt es hierbei aufgrund einer zu weit medialen Punktion nach der Portimplantation zu einer Einengung und repetitiven Kompression des Portschlauches in der kostoklavikulären Enge.

Klinisch fällt ein Pinch-off-Syndrom durch Armposition abhängige Infusionsflüsse und durch die typische Einengung im Röntgenbild in der kostoklavikulären Enge auf. Bei seitlicher Armposition kommt es zur Lumen-Einengung des Portschlauches in der kostoklavikulären Enge. Wenn die Schulter angehoben oder der Arm über Kopf gehoben wird, verschwindet die Lumen-Einengung. In Konsequenz sollten Röntgen-Aufnahmen bei Portpatienten im p-a-Strahlengang nicht mit nach vorne gerollten Armen aufgenommen werden, sondern mit seitlicher Armposition. Beim Pinch-off-Syndrom werden folgende Stadien unterschieden:

0. Normal.

1. Abrupte Unterbrechung im Verlauf ohne Einengung.

2. Lumen-Einengung.

3. Schlauchfraktur [12].

In einer Studie mit 987 Patienten wurde das Pinch-off-Syndrom in 11 Fällen beobachtet $(1,1 \%)$ :

- 8 innerhalb von 3 Wochen nach Implantation (73\%),

- 3 verspätet nach 12, 24, und 37 Wochen (27 \%),

- 1 Katheterfraktur nach 5 Wochen [12].

Das Pinch-off-Syndrom kam in unserem Patientenkollektiv weder als Früh- noch als Spätkomplikation vor. 


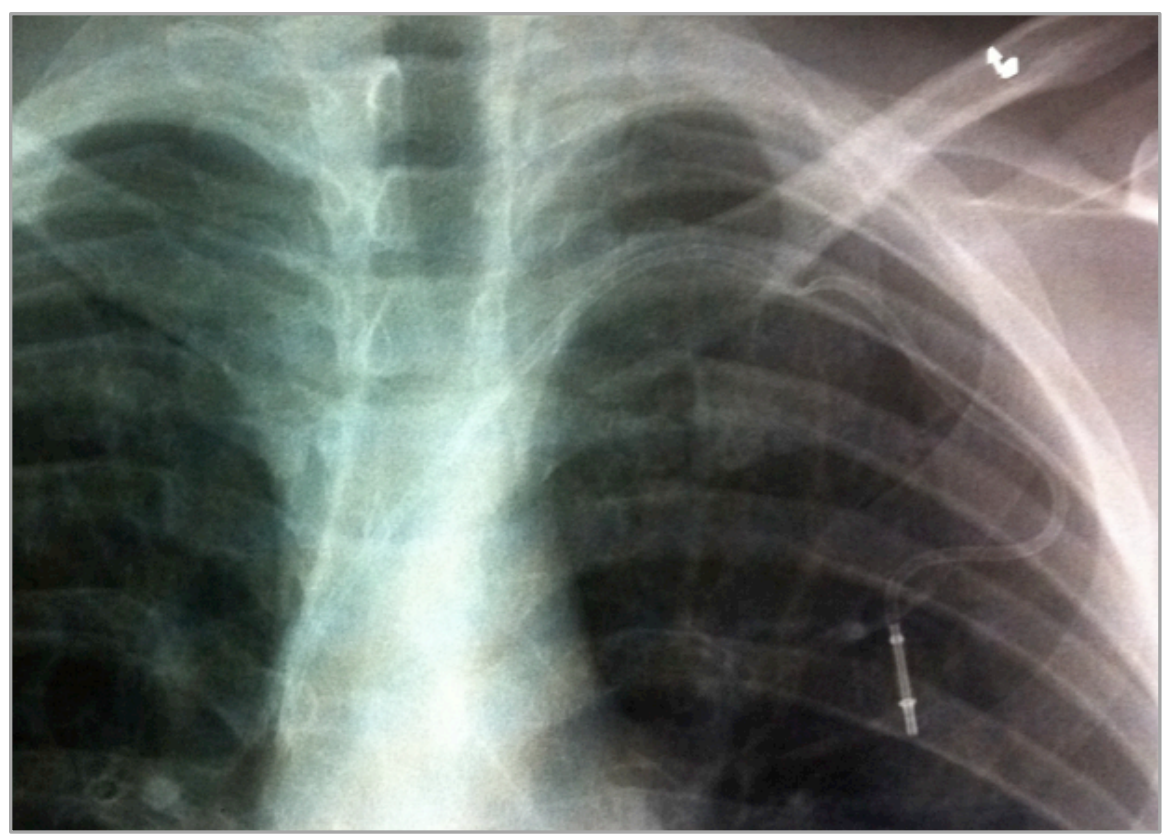

Abbildung 42: Ausschnitt aus einem Röntgenbild mit Einengung des Portschlauches in der kostoklavikulären Enge.

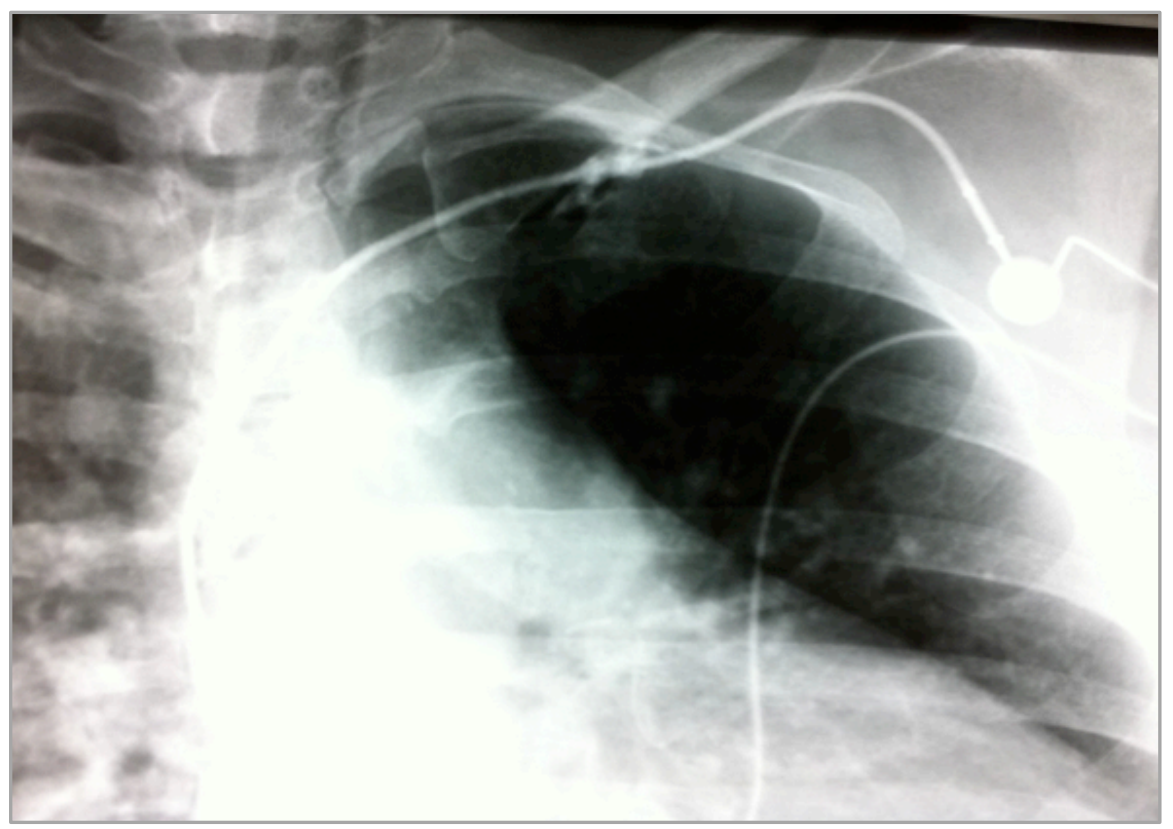

Abbildung 43: Durchleuchtungsaufnahme bei KM-Gabe über die Portkammer: Pinch-off mit KM-Austritt aus dem Portschlauch. 


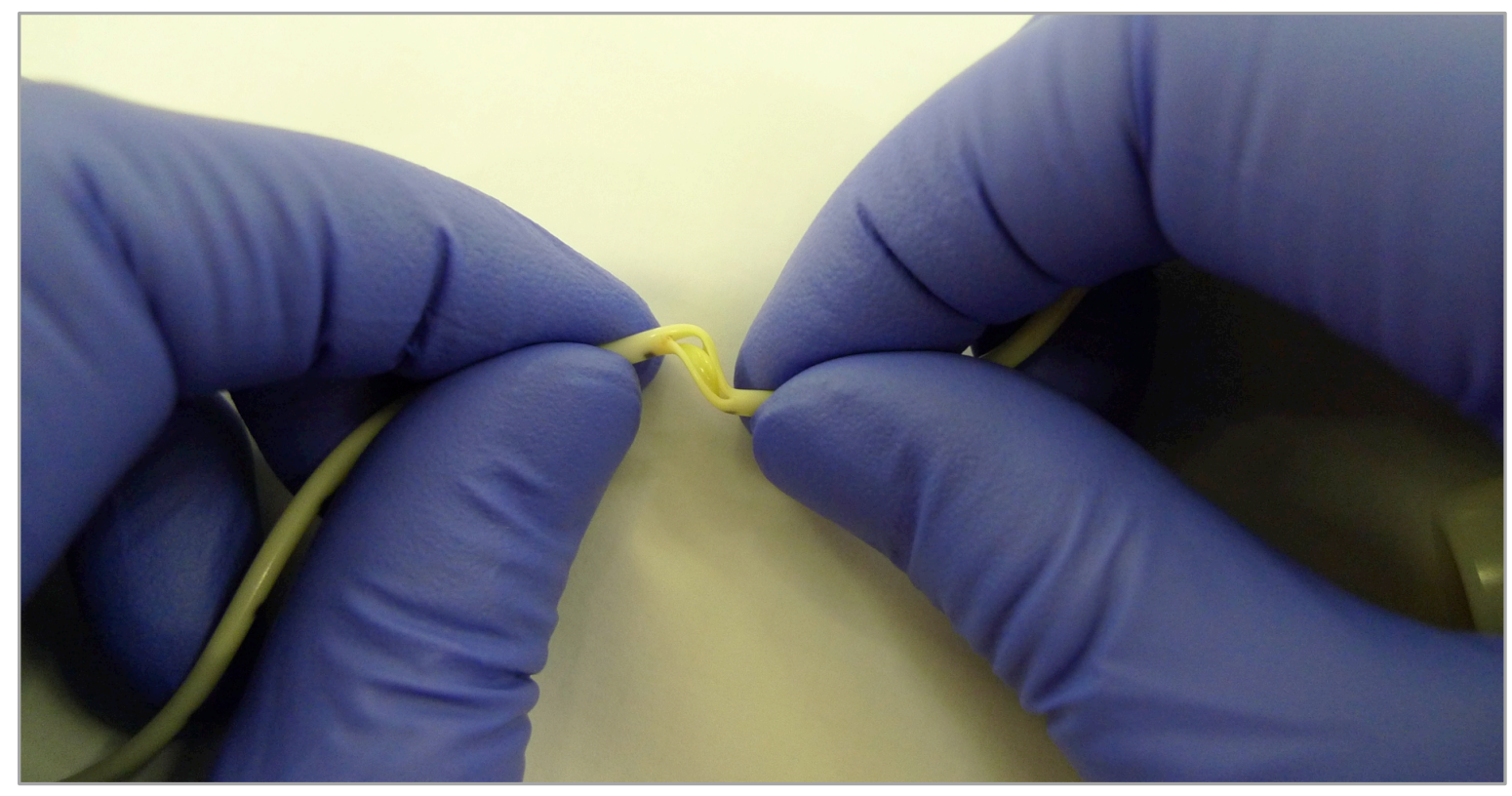

Abbildung 44: Explantierter Portschlauch bei Pinch-off mit Schlauchdefekt.

Das Pinch-off-Syndrom im Stadium 1 und 2 fällt klinisch durch höhere notwendige Drücke, um eine Infusion zu gewährleisten, auf. Langfristig führt es zu einem Stadium 3 mit Schlauchfraktur und Paravasat. Hier können bei der Chemotherapie lokale Nekrosen durch das Paravasat auftreten. In allen drei Stadien ist eine komplette Entfernung des Portkathetersystems notwendig. Der neue Portschlauch sollte hierbei nicht über einen Draht ausgetauscht werden, da so die alte Problematik des zu weit medialen Zugangs mit dem Risiko einer Einklemmung in der kostoklavikulären Enge weiterhin bestehen bleibt. Es ist eine erneute Punktion der V. subclavia weiter lateral notwendig. 
Mit einem $p$-Wert von 0,021 ergibt sich ein signifikanter Wert zu Gunsten der radiologischen Portimplantationen bei den Spätkomplikationen.

\section{Spätkomplikationen Chirurgie $(n=173)$}

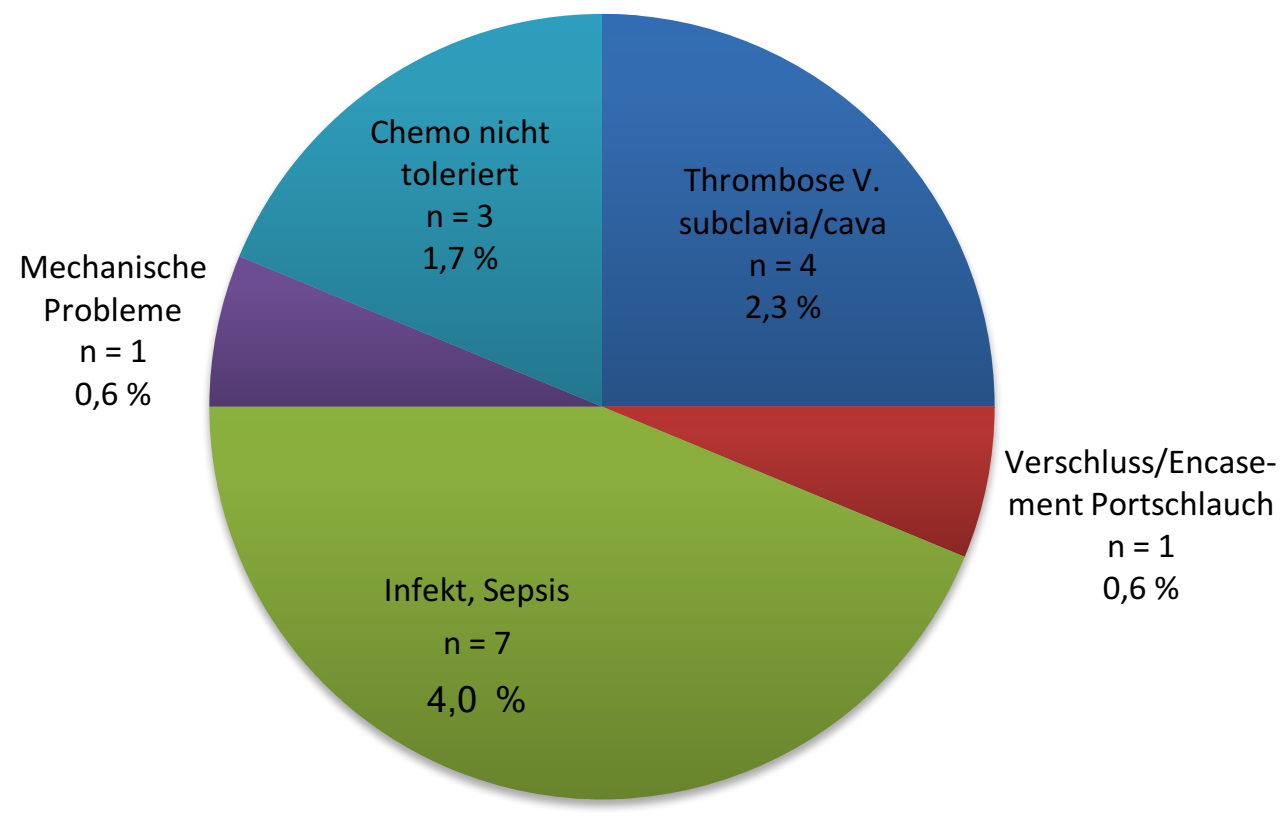

Abbildung 45: Spätkomplikationen in der Chirurgie bei insgesamt 173 Portimplantationen. 


\title{
Spätkomplikationen Radiologie $(n=554)$
}

\author{
Mechanische \\ Probleme \\ $\mathrm{n}=3$ \\ $0,5 \%$
}



Abbildung 46: Spätkomplikationen in der Radiologie bei insgesamt 554 Portimplantationen.

Mit einem $p$-Wert von 0,21 besteht eine signifikant geringere Komplikationsrate der radiologischen Spätkomplikationen versus chirurgischen. 


\section{Relative Häufigkeiten Spätkomplikationen Chirurgie}



Abbildung 47: Relative Häufigkeiten Spätkomplikationen in der Chirurgie.

\section{Relative Häufigkeiten Spätkomplikationen Radiologie}



Abbildung 48: Relative Häufigkeiten Spätkomplikationen in der Radiologie. 


\subsubsection{PORTSPÄTINFEKTION}

Ein signifikanter Unterschied in den Spätinfektionen war nicht zu erwarten, da beide Patientengruppen nach der Portimplantation im onkologischen Hause weiter betreut wurden. Die Portverwendung und Pflege sollte in beiden Patientengruppen identisch sein. Jedoch kam es in der Chirurgischen Klinik in 4 \% der Fälle zu Spätinfektionen vs. 2,9 \% in der radiologischen Gruppe.

Bei einer Portinfektion können klassische Entzündungszeichen wie Rötungen, lokale Schmerzen und Schwellungen entstehen. Andererseits können diese klassischen Entzündungszeichen komplett fehlen und der Patient zeigt nach der Verwendung der Portkammer ein septisches Bild mit Fieber. In beiden Fällen resultiert eine Explantation des kompletten Portsystems. Das Portkammersystem wird für eine weitere mikrobiologische Untersuchung in ein steriles Gefäß eingebracht. Die Portkammer wird mit Braunol ${ }^{\circledR}$ ausgewaschen. Im Falle eines Abszess-Verhaltes wird zusätzlich eine Minidrainage für einen Tag eingelegt. Sollte ein weiterer Portgebrauch notwendig sein, kann auf der kontralateralen Seite am gleichen Tag oder später eine neue Portimplantation erfolgen.

Mit einer relativen Häufigkeit von $44 \%$ an den Gesamtspätkomplikationen ist in beiden Gruppen die Portinfektion die häufigste Spätkomplikation.

4.3.2 VENENTHROMBOSE UND PORTSCHLAUCH-ENCASEMENT

Die Venenthrombose ist mit einer relativen Häufigkeit an den Gesamtspätkomplikationen in der Chirurgie mit $25 \%$ die zweithäufigste und in der Radiologie mit $17 \%$ die dritthäufigste Spätkomplikation. Durch eine Venenthrombose entsteht per se keine Beeinträchtigung des Portgebrauches. Je nach Klinik des Patienten erfolgt eine Heparinisierung des Patienten. Bei ausgeprägter Venen-Thrombosierung mit klinischer Beeinträchtigung sollte eine Explantation des Portsystems erwogen werden. 
Das Encasement des Portschlauches stellt in der Radiologie mit einer relativen Häufigkeit von $31 \%$ aller Spätkomplikation die zweithäufigste Spätkomplikation dar, mit nur $6 \%$ ist es in der Chirurgie nur die vierthäufigste Spätkomplikation.

Das Encasement tritt als einzige Komplikation häufiger im Radiologischen Institut - mit 2,0 $\%$ vs. $0,6 \%$ - als in der Chirurgischen Klinik auf. Dies kann durch die längere Verlaufsstrecke zur oberen Hohlvene des von links eingebrachten Portkatheters im radiologischen Verfahren gegenüber der kürzeren Verlaufsstrecke des von rechts eingebrachten chirurgischen Portkatheters zusammenhängen. Andererseits fehlt bei der chirurgischen Implantation ein direktes Trauma der V. subclavia. Der Portschlauch wird über eine kleinere, in die $\mathrm{V}$. subclavia mündende Vene eingeführt.

Wie in der Abbildung des Encasements (Abb. 38) in der Frühkomplikation zu sehen, verhindert die Fibrinumscheidung des Portschlauches den direkten Abstrom des Kontrastmittels oder der Infusion. Das Kontrastmittel läuft in der Fibrinumscheidung am Portschlauch nach kranial und tritt erst am Ende der Fibrinumscheidung in die V. cava superior. Zudem sind höhere Drücke notwendig und eine Infusion eines Chemotherapeutikums nicht oder nur mit deutlich höherem Zeitaufwand möglich. Zumeist ist zusätzlich der Portschlauch leicht nach kranial disloziert. Die Therapie in diesem Fall wäre - wie in der Frühkomplikation - eine Urokinase-Lyse über einen Perfusor durchzuführen (250000 IE Urokinase auf $50 \mathrm{ml} \mathrm{NaCl}$ über $5 \mathrm{~h}$ ). Anschließend erfolgt eine Kontrolle unter Durchleuchtung mit Kontrastmittel-Applikation über die Portkammer. Eine Blutaspiration über das Portkammersystem zeigt ebenfalls eine erfolgreiche Lyse an. Diese sollte auch mit einer Portographie dokumentiert werden. Bei noch bestehendem Rest-Encasement kann eine Wiederholung nach dem gleichen Schema erfolgen. Bei ausbleibendem Erfolg muss eine Revision des Portschlauches in Erwägung gezogen werden.

Bei asymptomatischen Patienten $(n=57)$ wurden in einer Studie nach Venographie inkomplette Thromboseraten der V. subclavia von 45,5\% und komplette Verschlüsse der V. subclavia, ebenfalls asymptomatisch, von 10,5\% aufgezeigt. Die Encasement-Rate lag in der gleichen Studie bei 78 \%. Es gab keinen signifikanten Unterschied zwischen langen und kurzen zentralvenösen Kathetern [1]. 
Alternativ zur Encasement-Lyse kann ein perkutanes Stripping des Encasements durchgeführt werden. In einer Studie konnte jedoch kein signifikanter Unterschied im Vergleich zu einer Urokinase-Lyse (250 000 IE, 30000 IE / h) gezeigt werden [7].

\subsubsection{MECHANISCHE SPÄTKOMPLIKATIONEN}

Die mechanischen Spätkomplikationen nehmen mit einem relativen Anteil von $6 \%$ in der Chirurgie und $8 \%$ in der Radiologie jeweils den geringsten Anteil der Spätkomplikationen ein. Wie in der Beschreibung der Frühkomplikationen erwähnt, können hier auftreten: Portmembrandefekt, Defekte des Portschlauches portnah oder im mittleren Anteil (Pinchoff-Syndrom). Die Konsequenz der genannten mechanischen Komplikationen ist die Explantation des Portsystems. Eine weitere mechanische Komplikation ist ein kraniales Umschlagen des distalen Portschlauches. Bei dieser Komplikation besteht über einen venösen Zugang die Möglichkeit der transfemoralen Korrektur mittels Katheter (Abb.: 48 $50)$.

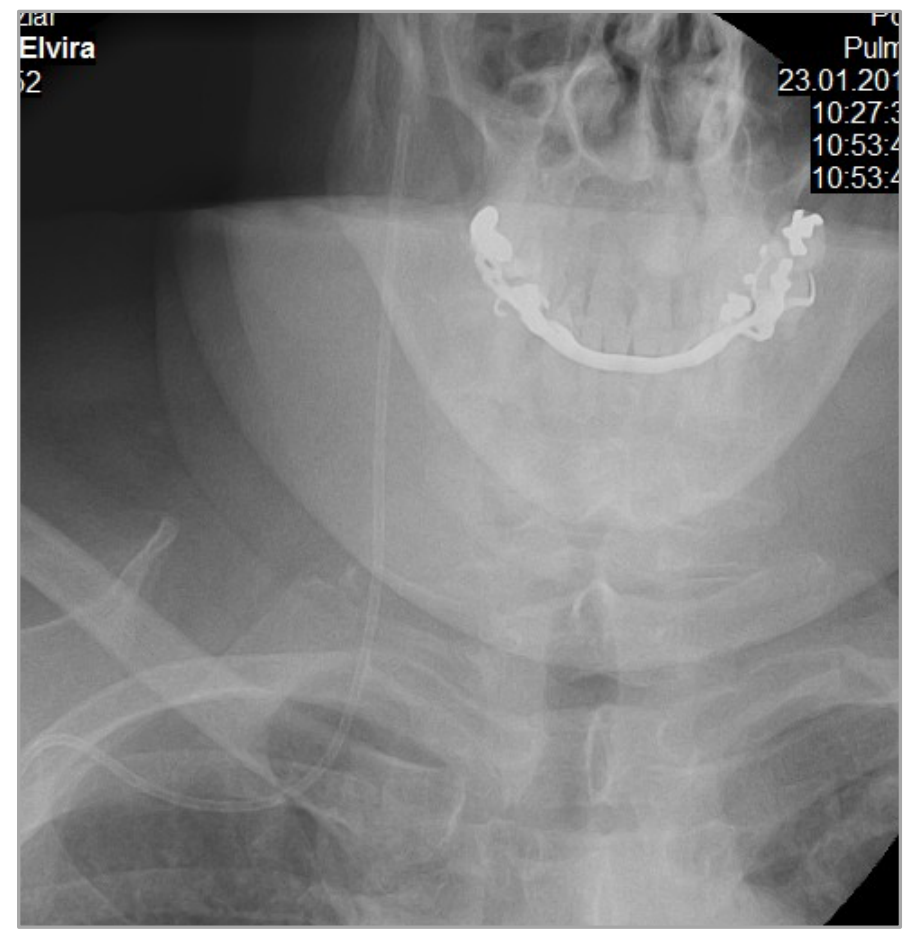

Abbildung 49: Nach kranial in die V. jugularis interna rechts umgeschlagener Portschlauch. 

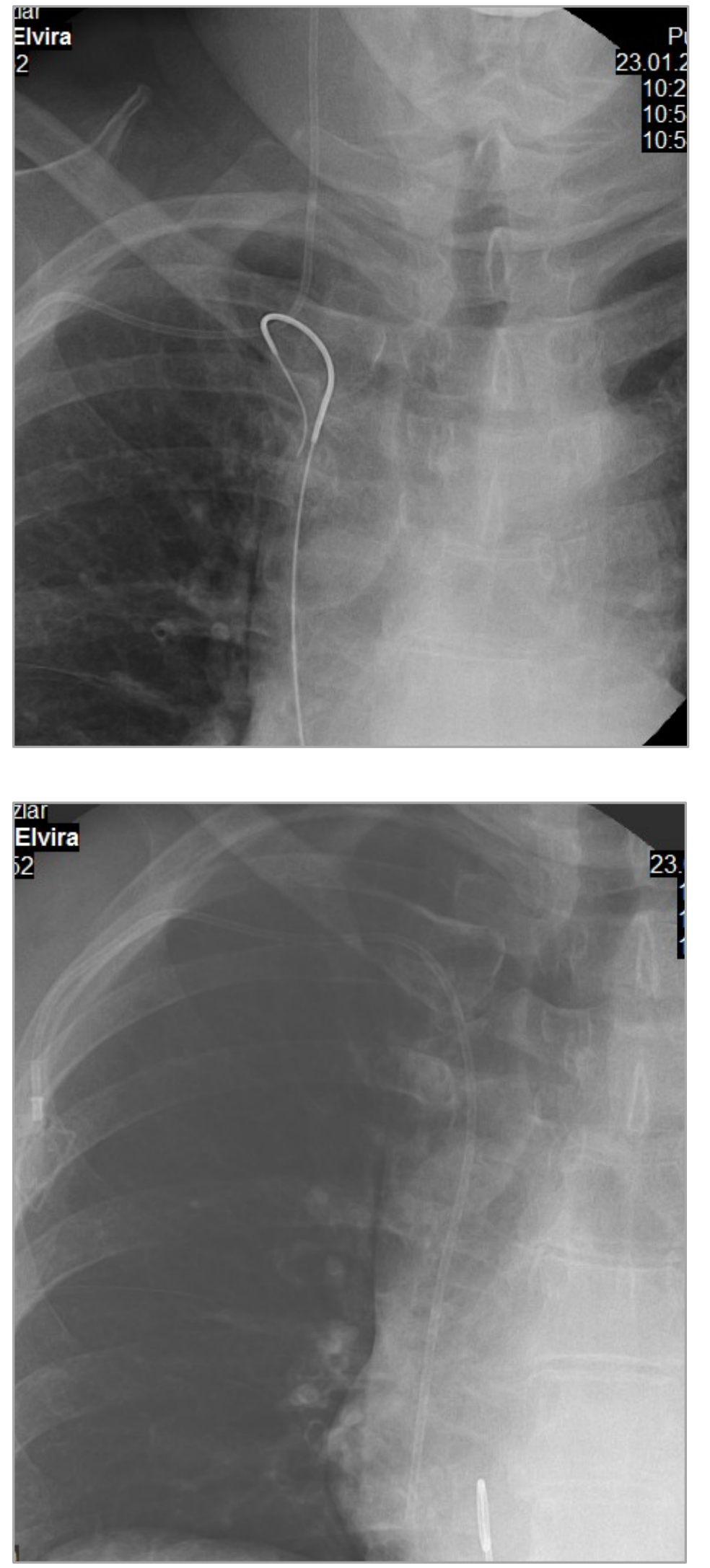

Abbildung 50: Über venösen Zugang transfemorales Einfangen des umgeschlagenen Portschlauches mit einem 5F Pigtailkatheter und Führungsdraht.

\footnotetext{
Abbildung 51: Nach erfolgreicher Reposition des Portschlauches in die V. cava superior.
} 




Abbildung 52: Explantierter Port mit gelöstem Klickkonnektor und perforiertem Schlauch.

Abb. 51 zeigt einen radiologisch explantierten Port eines chirurgischen Patienten, ein Seltener Fall eines mechanischen Defektes - bei nicht fixierter Muffe kam es zur Portschlauch-Perforation.

\subsubsection{SONSTIGE KOMPLIKATIONEN}

Mit $19 \%$ fiel bei den chirurgischen Spätkomplikationen die Unverträglichkeit der Chemotherapie ins Gewicht. In der radiologischen Gruppe wurde eine Portexplantation aufgrund einer nicht tolerierten Chemotherapie nicht beobachtet. 


\section{ZUSAMMENFASSUNG}

Sichere, langfristige und zuverlässige Medikamentenapplikationen sind essentieller Bestandteil der Chemotherapie. Hierfür werden die Patienten mit einem Port versorgt.

In mehreren Studien werden chirurgische und radiologische Ports bezüglich ihrer Frühund Spätkomplikationen verglichen, jedoch werden meist verschiedene venöse Zugänge für die Ports genutzt (V. subclavia, V. jugularis interna). In unserer retrospektiven Studie wurden chirurgische und radiologische Implantationsverfahren über den Zugang der rechten V. cepahlica mittels Cutdown (chirurgische Implantation) und Punktion der linken V. subclavia (radiologische Implantation) verglichen. Dies stellt eine gute Vergleichsmöglichkeit bei venösen Zugängen im gleichen Zugangsgebiet dar. Trotz der verfahrensbedingten radiologischen Komplikationsmöglichkeiten des Pneumothoracis und des Pinch-off-Syndroms schneidet die radiologische Implantation in den Früh- und Spätkomplikationen signifikant besser ab (Frühkomplikation $p=0,022$, Spätkomplikation $p$ = 0,021). Die Gesamtpneumothoraxrate lag bei insgesamt 554 Portimplantationen bei 0,9 $\%(n=5)$. Die Komplikation des Pinch-off-Syndroms wurde in unserem radiologischem Patientenkollektiv bei lateraler Punktion der V. subclavia nicht beobachtet.

Der Vergleich des Durchmessers der Punktionsnadel (21 $\mathrm{G}=0,8 \mathrm{~mm}$ vs. $18 \mathrm{G}=1,3 \mathrm{~mm}$ ) erbrachte keinen signifikanten Unterschied. Jedoch kam es bei insgesamt 84 Portimplantationen - durchgeführt von einem Interventionsanfänger - zu keinem Pneumothorax. Eine prospektiv-randomisierte Studie wäre notwendig um ggf. einen Unterschied des Pneumothoraxrisikos in Abhängigkeit vom Durchmesser der Punktionsnadel zu zeigen.

Die Präferenz zur Portimplantation über die V. jugularis interna wird in Studien u. a. mit dem geringeren Pneumothoraxrisiko begründet. In einer großen Metaanalyse konnte jedoch kein Unterschied in der Inzidenz von Hämato- oder Pneumothorax und Gefäßverschlüssen gezeigt werden. Es kam über den Zugang der V. jugularis interna zu vermehrt arteriellen Fehlpunktionen, jedoch weniger Fehlplatzierungen verglichen mit dem Zugang via V. subclavia [22].

Insgesamt ist die bildgesteuerte, radiologische Portimplantation mittels Punktion der V. subclavia ein sicheres Verfahren. 
Safe, long-term and reliable drug applications are essential for chemotherapies. For this, patients are supplied with ports.

In several studies surgical and radiological ports are compared with respect to their late complications, in most cases not taking into account the different venous accesses of the ports.

The retrospective study presented here compares surgical port implantation by cutdown (V. cephalica, $n=173$ ) vs. radiological implantation (left V. subclavia, $n=554$ ) in terms of early and late complications, thereby providing for a more accurate comparability. Despite the possible additional complications regarding the radiological procedure (pneumothorax and Pinch-off-Syndrom), this method showed significantly fewer early ( $p=0.022)$ and late complications $(p=0.021)$. Within a total of 554 port implantations, the overall rate of pneumothraces was $0.9 \%(n=5)$. Regarding lateral puncture of the V. subclavia, the Pinch-off-Syndrom was not observed in the group of patients treated.

A comparison of the diameter of the puncture needle $(21 \mathrm{G}=0.8 \mathrm{~mm}$ vs. $18 \mathrm{G}=1.3 \mathrm{~mm}$ ) showed that this variable was not of significant influence. There were no pneumothoraces in 84 port implantations performed by an interventional beginner (18 G puncture needle). In order to analyze differences in the risk of a pneumothorax, a prospective randomized study would be needed.

Many studies justify the preference of port implantation via the $\mathrm{V}$. jugularis interna by referring to the lower risk of pneumothorax. Yet, no difference in the incidence of hämatoand pneumotaracis could be shown by a meta-analysis. There were more arterial malocclusions but fewer misplacements compared to implantations via the $\mathrm{V}$. subclavia [22].

Overall, the image-guided, radiological port implantation by puncture of the V. subclavia is a safe procedure. 


\section{ABBILDUNGSVERZEICHNIS}

Abbildung $1 \quad$ Kunststoff-Portsystems der Firma Bard, bestehend aus: 1) Portkammer, 2) Klick-Konnektor, 3) Portschlauch.

Abbildung 2 Implantierte Portkammer im Querschnitt.

Abbildung 3 Portbesteck, Bard Power Plus ${ }^{\circledR}$.

Abbildung 4 Aufgrund eines Schrägschliffs nicht stanzende Hubernadel aus einem Portset der Firma Bard®.

Abbildung 5 Schwarze Linien: Hauptbronchus rechts; Horizontale, rote Linien: Wirbelkörperdeckplatten, in Anlehnung an: PierreYves Marcy Eur Radiol (2008) 18: 2333-2344 [17].

Abbildung 6 Links: Schnittführung und Platzierungsmöglichkeiten der Portkammer bei Implantation venöser Portsysteme. Rechts: Schematische Darstellung des Venenverlaufes im SchulterHals-Bereich, aus: Haindl H, Müller H, Schmoll E, Hrsg.: Portkathetersysteme. Heidelberg: Springer; 1993: 25; With permission of Springer [8].

Abbildung 7 Links: V. cephalica angeschlungen und eröffnet. Rechts:

Einführen des Katheter in die V. cephalica. Intraoperativer Situs, aus: Haindl $H$, Müller $H$, Schmoll E, Hrsg.:

Portkathetersysteme. Heidelberg: Springer; 1993: 27; With permission of Springer [8].

Abbildung 8 Beispiel einer Venographie rechts und links, jeweils mit einliegendem 0,018 Draht. 
Abbildung $921 \mathrm{G}$ Micropuncturenadel mit $10 \mathrm{ml}$ Lokalanästhesie.

Abbildung 10 Durchführung der Lokalanästhesie mit Doppelwandpunktion

Abbildung 11 Rückzug und Blutaspiration.

Abbildung 12 Einführen des 0,018“ Drahtes durch die Micropuncturenadel.

Abbildung 13 Vergleich (oben, grün), Micropuncturenadel $21 \mathrm{G}$ der Firma

CookMedical mit einem Außendurchmesser von 0,8 mm, (unten rosa) normale Punktionsnadel $18 \mathrm{G}$ aus dem

Portbesteckset der Firma Bard mit einem Außendurchmesser von $1,3 \mathrm{~mm}$.

Abbildung 14 Micropunctureset der Firma CookMedical, USA, bestehend aus (von oben nach unten):

1. Zweiteilige Minischleuse, $5,0 \mathrm{Fr} / 10 \mathrm{~cm}$. 2. $21 \mathrm{G}$ Punktionskanüle. 3. 0,018“ Führungsdraht / $40 \mathrm{~cm}$.

Abbildung 15 Punktion der V. subclavia mittels Feinnadel und eingeführtem 0.018“ Draht.

Abbildung 16 Verifizierung der venösen Punktion; der Führungsdraht liegt rechts paravertebral unterhalb des Zwerchfells in der V. cava inferior.

Abbildung 17 Austausch des Feinnadelsets über einen 0.035“ Draht gegen eine Peel-away-Schleuse.

Abbildung 18 Situs mit subkutaner Porttasche und Tunnelung mittels Moskitoklemme. 
Abbildung 19 Nach Einführen des Portschlauches. Die Peel-away-Schleuse wird aufgespalten und entfernt.

Abbildung 20 Situs nach Entfernen der Peel-away-Schleuse mit venös einliegendem Portschlauch.

Abbildung 21 Platzieren der Portkammer in der subkutanen Porttasche.

Abbildung 22 Nichtresorbierbare Einzelknopfnaht mit Portnadel mit 21 Funktionskontrollen (Blutaspiration und Spülen).

Abbildung 23 Start der Nadel im subkutanen Fettgewebe und Herausführen im subkutanen Bindegewebe.

Abbildung 24 Auf der Gegenseite Durchzug der Nadel aus dem subcutanen Bindegewebe in das subcutane Fettgewebe. Beide Fadenenden werden nach oben angezogen.

Abbildung 25 Abschlussskizze nach Knoten.

Abbildung 26 Beispiel einer nicht resorbierbaren Einzelknopfnaht (nach Donati).

Abbildung $27 \quad$ Beispiel Hautverhältnisse nach subkutaner Einzelknopfnaht.

Abbildung 28 Zugang zur V. cava superior über Umgehungskreisläufe bei Verschluss der V. subclavia.

Abbildung 29 Persistierende obere Hohlvene. Nach Einlage des Portschlauches fiel der atypische Verlauf desselbigen auf. Nach Kontrastmittel-Gabe und Venographie fand sich eine persistierende obere Hohlvene. 
Abbildung 30 Die zehn häufigsten Krebs-Neuerkrankungen, aus: 30 Hessisches Krebsregister 2008 [11].

Abbildung 31 Chirurgie - Tumorentitäten.

Abbildung 33 Auswärts eingelegter Port über die V. jugularis interna. Der

Portschlauch wird über die Klavikula getunnelt und demarkiert sich unter der Haut.

Abbildung 34 Gleiche Patientin wie in Abb. 32: Auf Wunsch der Patientin Entfernung des links seitigen Jugularis interna Ports und Implantation über die $\mathrm{V}$. subclavia rechts.

Abbildung 35 Frühkomplikationen in der Chirurgie

Abbildung 37 Relative Häufigkeiten Frühkomplikationen in der Chirurgie.

Abbildung 38 Relative Häufigkeiten Frühkomplikationen in der Radiologie. aufgrund der Fibrin-Umscheidung des Portschlauches und tritt am Ende der Fibrin-Umscheidung in die V. cava superior über. Häufiger Nebenbefund beim Encasement ist, wie auch hier, ein nach kranial dislozierter Portschlauch.

Abbildung 40 Gleiche Patientin wie in Abb. 38 nach erfolgreicher Lyse. Unauffälliger KM-Abstrom, sowie spontan nach kaudal repositionierter Portschlauch. 
Abbildung 41 Anderer Pat. mit ausgeprägtem Encasement. Um den Portschlauch ausgeprägter Thrombus in koronarer Rekonstruktion.

Abbildung 42 Ausschnitt aus einem Röntgenbild mit Einengung des

Portschlauches in der kostoklavikulären Enge.

Abbildung 43 Durchleuchtungsaufnahme bei KM-Gabe über die Portkammer: Pinch-off mit KM-Austritt aus dem Portschlauch.

Abbildung 44 Explantierter Portschlauch bei Pinch-off mit Schlauchdefekt.

Abbildung 45 Spätkomplikationen in der Chirurgie bei insgesamt 173 Portimplantationen.

Abbildung 46 Spätkomplikationen in der Radiologie bei insgesamt 554 Portimplantationen.

Abbildung 47 Relative Häufigkeiten Spätkomplikationen in der Chirurgie.

Abbildung 48 Relative Häufigkeiten Spätkomplikationen in der Radiologie.

Abbildung 49 Nach kranial umgeschlagener Portschlauch.

Abbildung 50 Über venösen Zugang transfemorales Einfangen des umgeschlagenen Portschlauches mit einem 5F Pigtailkatheter.

Abbildung $51 \quad$ Nach erfolgreicher Repositon des Portschlauches in die V. cava superior.

Abbildung 52 Explantierter Port mit gelöstem Klickkonnektor und 66 perforiertem Schlauch. 


\section{TABELLENVERZEICHNIS}

Tabelle 1 Gesamtzahl der Portimplantationen innerhalb von zwei Jahren, aufgeteilt nach Abteilungen.

Tabelle 2 Häufigkeit der Portimplantationen nach stationär / ambulant.

Tabelle 3 Häufigkeiten nach Geschlecht.

Tabelle 4 Anteil der Patienten mit Metastasierung bei

Portimplantation nach Abteilung.

Tabelle 5 Portimplantationen durchgeführt von Facharzt oder Assistenzarzt.

Tabelle $6 \quad$ Verwendete Porthersteller in der Radiologischen Abteilung.

Tabelle $7 \quad$ Klinisch apparente Komplikationen innerhalb von 30 Tagen.

Tabelle $8 \quad$ Klinisch apparente Komplikationen nach 30 Tagen.

Tabelle 9 Frühkomplikationen Pneumothorax Radiologie. 


\section{GRÖßENANGABEN}

\section{Gauge (G):}

Die Einheit steht bei Nadel und Kanülen für den Außendurchmesser, der umso geringer ist, je höher der Wert ist. Die Einheit ist der amerikanischen Einheit für Drähte entlehnt, bei der die Zahl des G-Wertes der Zahl der Arbeitsgänge entspricht, d.h. je häufiger ein Draht durch die Drahtziehmaschine läuft, desto dünner wird er. Die Umrechnung in Millimeter und die Farbkodierung sind in ISO 6009, DIN 13095 und ISO/DIN 9626 festgehalten.

\section{French $(\mathrm{F})$ oder Charrière $(\mathrm{CH})$ :}

Die Einheit steht in der Angiographie für den Außendurchmesser von Kathetern und für den Innendurchmesser von Schleusen.

$1 \mathrm{~F}=1 \mathrm{CH}=0,33 \mathrm{~mm}$.

Inch (in, "):

Der Außendurchmesser von Führungsdrähten wird in Inch angegeben, das gleichzusetzen ist mit dem internationalen oder englischen Zoll.

$1 \mathrm{in}=1 \mathrm{Zoll}=25,4 \mathrm{~mm}$

aus:

Reithoffer M, Grillenberger A: Angiographie in Theorie und Praxis. Facultas, 2009: 63. [21] 
A

Abb.

bzw.

ca.

CA

d

evtl.

G

GIT

ggf.

GYN

$\mathrm{h}$

$\mathrm{HNO}$

IE

INR-Wert
Arteria

Abbildung

beziehungsweise

circa

Karzinom

Tage

Eventuell

Gauge

Gastrointestinaltrakt

Gegebenenfalls

Gynäkologie

Stunden

Hals-Nasen-Ohren

Internationale Einheit

Inaternational Normalized Ratio 
$\mathrm{KM}$

MTA

$\mathrm{NaCl}$

OP

p.a.

Pat.

PUR

TIVAD

rtPA

TU

u.a.

URO

V.

vs.

ZVK

z.B.
Kontrastmittel

Medizinisch-technische/r Assistent/in

Natrium Chlorid

Operation

posterior-anterior

Patient

Polyurethan

Totally Implantable Acces Device

Recombiant tissue plasminogen activator

Tumor

unter anderem

Urologie

Vena

versus

Zentraler Venenkatether

zum Beispiel 


\section{LITERATURVERZEICHNIS}

1. Balestreri L, De Cicco M, Matovic M, Coran F, Morassut S: Central venous catheter-related thrombosis in clinically asymptomatic oncologic patients: a phlebographic study. European Journal of Radiology, 1995. 20(2): 108 - 111.

2. Brant-Zawadzki M, Anthony M, Mercer EC: Implantation of P. A. S. port venous acces device in the forearm under fluoroscopic guidance. American Journal of Roentgenology, 1993. 160: 1127 - 1128.

3. Collignong P, Soni N, Perason I, Sorrel T, Woods P: Sepsis associated with central vein catheters in critically ill patients. Intensive Care Medicine, 1988, 14(3): 227 231.

4. Deitscher SR, Fesen MR, Kiproff PM, Hill PA, Li X, McCluskey ER, et al: Safety and efficacy of alteplase for restoring function in occluded central venous catheters: results of the cardiovascular thrombolytic to open occluded lines trial. Clinical Oncology, 2002. 20(1): 317 - 324.

5. Forssmann W: Die Sondierung des rechten Herzens. Klinische Wochenschrift 1929. 8(45): $2085-2087$.

6. Granziera E, Scarpa M, Ciccarese A, Filip B, Cagol M, Manfredi V, et al: Tatally implantable venous access devices: retrospective analysis of different insertion techniques and predictors of complications in 796 devices implanted in a single institution. BMC Surgery, 2014. 14: 27.

7. Gray RJ, Levitin A, Buck D, Brown LC, Sparling YH, Jablonski KA: Percutaneous fibrin sheath stripping versus transcatheter urokinase infusion for malfuncitoning well-positioned tunneled central venous dialysis catheters: a prospective, randomized trial. Journal of Vascular and Interventional Radilogy, 2000. 11(9): $1121-1129$.

8. Haindl H, Müller H, Schmoll E, Hrsg.: Portkathetersysteme. Praxisnahe Information zu Indikationen, Implantionstechnik, Handhabung. Heidelberg: Springerverlag, 1993: 25, 27.

9. Henriques HF 3rd, Karmy-Jones R, Knoll SM, Copes WS, Giordano JM: Avoiding complications of long-term venous acces. The Amercian Surgeon, 1993. 53(9): 555 $-558$. 
10. Herrmann KA, Waggershauser T, Helmberger T, Heinemann V, Sittek H, Reiser M: Internventionell-radiologische perkutane Implantation intravenöser Port-KatheterSystem. Der Radiologe, 1999. 39(9): 777 - 782.

11. Hessisches Sozialministerium: Krebs in Hessen. Inzidenz und Mortalität 2008.

Wiesbaden, 2012: 21.

https://www.laekh.de/images/Die Kammer/Hessisches Krebsregister/Krebsbericht 2008.pdf, Zugegriffen: 12. Februar 2017.

12. Hinke DH, Zandt-Stastny DA, Goodman LR, Quebbeman EJ, Krzywda EA, Andris DA: Pinch-off syndrome: a complication of implantable subclavion venous acces devices. Radiology, 1990. 177(2): 353 - 356.

13. Kluge A, Rauber K, Rau WS: Radiologisch implantierte subkutan getunnelte zentrale Venenkatheter zur Dialyse: Verlauf und Komplikationen. RöFo, 1997. 166 (3): $230-232$.

14. Kluge A, Stroh H, Wagner D, Rauber K: Durchleuchtungsgesteuerte Implantation subkutaner Venenports: Komplikationen und Lanzeitergebnisse. RöFo, 1998. 169(7): $63-67$.

15. Kock HJ, Krause U, Pietsch M, Rasfeld S, Walz MK: Implantierbare Kathetersysteme: Erfahrungen bei 1000 Patienten mit zentralvenösen Ports. Dtsch Med Wochenschr, 1996. 121 (3): 47 - 51.

16. Laméris JS, Post PJ, Zonderland HM, Gerritsen PG, Kappers-Klunne MC, Schütte HE: Percutaneous placement of Hickman catheters: comparison of sonographically guided and blind techninques. American Journal of Roentgenology, 1990. 155(5): 1097 - 1099.

17. Marcy PY: Central venous access: techniques and indications in oncology. European Radiology, 2008. 18(10): 2333-2344.

18. Morris SL, Jaques PF, Mauro MA: Radiology-assisted placement of implantable subcutaneous infusion ports for long-term venous access. Radiology, 1992. 184(1): $149-151$.

19. Niederhuber JE, Ensminger W, Gyves JW, Liepman M, Doan K, Cozzi E: Totally implanted venous and arerial access system to replace external catheters in cancer treatment. Surgery, 1982. 92(4): 706 - 712.

20. Rathmann N, Hausmann D, Kostrzewa M, Keese M, Diel S, Schönberg M, et al: Komplikation venöser Portsysteme. Radiologische Diagnostik und minimalinvasive Therapie. Der Radiologe, 2011. 51(5): 397 - 404. 
21. Reithoffer M,Grillenberger A: Angiographie in Theorie und Praxis. Facultas, 2009: 63.

22. Ruesch S, Walder B, Tramèr MR: Complications of central venous catheters: internal jugular versus subcavian access-a systematc review. Critical Care Medicine, 2002. 30(2): 454 - 460.

23. Seldinger SI: Catheter Replacement of the Needle in Percutaneous Arteriography: A new technique. Acta Radiologica (Old Series), 1953. 39(5): 368 - 376.

24. Silberzweig JE, Sacks D, Khorsandi AS, Bakal CW: Reporting standards for cetral venous acces. Journal of Vascular and Interventional Radiology, 2003. (14): 443 452.

25. Teichgräber UKM, Benter T, Schultz HJ, KLuhs L, Gutberlet M, Felix R: Ultraschallgesteuerte Punktionstechnik von zentral-venösen Gefäßen in EinPersonen-Technik. Ulltraschall in Medizin, 2000. 21(3): 132 - 136.

26. Teichgräber UKM, Gebauer B, Benter T, Wagner J: Langfristige zentralvenöse Zugänge und deren Komplikationsmanagement. RöFo, 2004. 176(7): 944 - 952.

27. Teichgräber UKM, Pfitzmann UR, Hoffmann HAF: Portsysteme als integraler Bestandteil von Chemotherapien. Deutsches Ärzteblatt International, 2014. 2(1): 97.

28. Vardy J, Engelhardt K, Cox K, et al: Long-term outcome of radiological-guided insertion of implanted central venous access port devices (CVAPD) for the delivery of chemotherapy in cancer patients: institutional experience and review of literature. Br J Cancer, 2004. 91 (6):1045-105.

29. Wagner HJ, Teichgräber $U$, Gebauer $B$, Kalinowski $M$ : Die transjuguläre Implantation venöser Portkathetersysteme. RöFo, 2003. 175(11): 1539 - 1544.

30. Walser EM: Venous Access Ports: Indications, Implantation Technique, Follow-Up, and Complications. CardioVascular and Interventional Radiology, 2011. 35(4): 751 -764 .

31. Wenke K, Markewitz A Vollständig implantierbare Kathetersysteme: Langzeitergebnisse - Komplikationen. Fortschr Med, 1990. 108: 276 - 279.

32. Whigham CJ, Lindsey JI, Goodman CJ, Fisher RG: Venous Port Salvage Utilizing Low Dose tPA. CradioVascular and Interbentional Radiology, 2002. 25(6): 513 516. 
33. Yukisawa S, Fujiwara Y, Yammoto Y, Uneno T, Matsueda K, Kohno A, et al: Upper-extremity deep vein thrombosis related to central venous port systems implanted in cancer patients. The British Journal of Radiology, 2010. 83(994): 850 $-853$.

34. Zähringer M, Hilgers J, Krüger K, Strohe D, Bangard C, Neumann L, et al: Ultrasound guided implantation of chest port systems via the lateral subclavioan vein. RöFo, 2006. 178(3): $324-325$. 


\section{EHRENWÖRTLICHE ERKLÄRUNG ZUR DISSERTATION}

Hiermit erkläre ich, dass ich die vorliegende Arbeit selbständig und ohne unzulässige Hilfe oder Benutzung anderer als der angegebenen Hilfsmittel angefertigt habe. Alle Textstellen, die wörtlich oder sinngemäß aus veröffentlichten oder nichtveröffentlichten Schriften entnommen sind, und alle Angaben, die auf mündlichen Auskünften beruhen, sind als solche kenntlich gemacht. Bei den von mir durchgeführten und in der Dissertation erwähnten Untersuchungen habe ich die Grundsätze guter wissenschaftlicher Praxis, wie sie in der „Satzung der Justus-Liebig-Universität Gießen zur Sicherung guter wissenschaftlicher Praxis" niedergelegt sind, eingehalten sowie ethische, datenschutzrechtliche und tierschutzrechtliche Grundsätze befolgt. Ich versichere, dass Dritte von mir weder unmittelbar noch mittelbar geldwerte Leistungen für Arbeiten erhalten haben, die im Zusammenhang mit dem Inhalt der vorgelegten Dissertation stehen, oder habe diese nachstehend spezifiziert. Die vorgelegte Arbeit wurde weder im Inland noch im Ausland in gleicher oder ähnlicher Form einer anderen Prüfungsbehörde zum Zweck einer Promotion oder eines anderen Prüfungsverfahrens vorgelegt. Alles aus anderen Quellen und von anderen Personen übernommene Material, das in der Arbeit verwendet wurde oder auf das direkt Bezug genommen wird, wurde als solches kenntlich gemacht. Insbesondere wurden alle Personen genannt, die direkt und indirekt an der Entstehung der vorliegenden Arbeit beteiligt waren. Mit der Überprüfung meiner Arbeit durch eine Plagiatserkennungssoftware bzw. ein internetbasiertes Softwareprogramm erkläre ich mich einverstanden.
Ort, Datum
Unterschrift 


\section{DANKSAGUNGEN}

Ich danke besonders Herrn Prof. Dr. Klaus Rauber für die Hilfe bei der Auswahl des Themas und die ständige fachliche und moralische Unterstützung während aller Bearbeitungsphasen.

Außerdem danke ich Herrn PD Dr. Andreas Kluge für die konstruktive Kritik und seine methodischen und fachlichen Ratschläge.

Danke für die Ratschläge in der Korrektur und das Layout sowie die moralische Unterstützung durch viele Unwägbarkeiten an meine geliebte Frau Anne-Christine. 